Florida International University FIU Digital Commons

$3-25-2016$

\title{
Characterization of Homogenized Mechanical Properties of Porous Ceramic Materials Based on Their Realistic Microstructure
}

Siavash Rastkar

Florida International University, srast002@fiu.edu

DOI: 10.25148 /etd.FIDC000247

Follow this and additional works at: https://digitalcommons.fiu.edu/etd

Part of the Applied Mechanics Commons

\section{Recommended Citation}

Rastkar, Siavash, "Characterization of Homogenized Mechanical Properties of Porous Ceramic Materials Based on Their Realistic Microstructure" (2016). FIU Electronic Theses and Dissertations. 2478.

https://digitalcommons.fiu.edu/etd/2478 


\section{FLORIDA INTERNATIONAL UNIVERSITY \\ Miami, Florida}

\section{CHARACTERIZATION OF HOMOGENIZED MECHANICAL PROPERTIES OF POROUS CERAMIC MATERIALS BASED ON THEIR REALISTIC MICROSTRUCTURE}

A dissertation submitted in partial fulfillment of the requirements for the degree of DOCTOR OF PHILOSOPHY

in

MECHANICAL ENGINEERING

by

Siavash Rastkar 


\section{To: Interim Dean Ranu Jung}

College of Engineering and Computing

This dissertation, written by Siavash Rastkar, and entitled Characterization of Homogenized Mechanical Properties of Porous Ceramic Materials Based on Their Realistic Microstructure, having been approved in respect to style and intellectual content, is referred to you for judgment.

We have read this dissertation and recommend that it be approved.

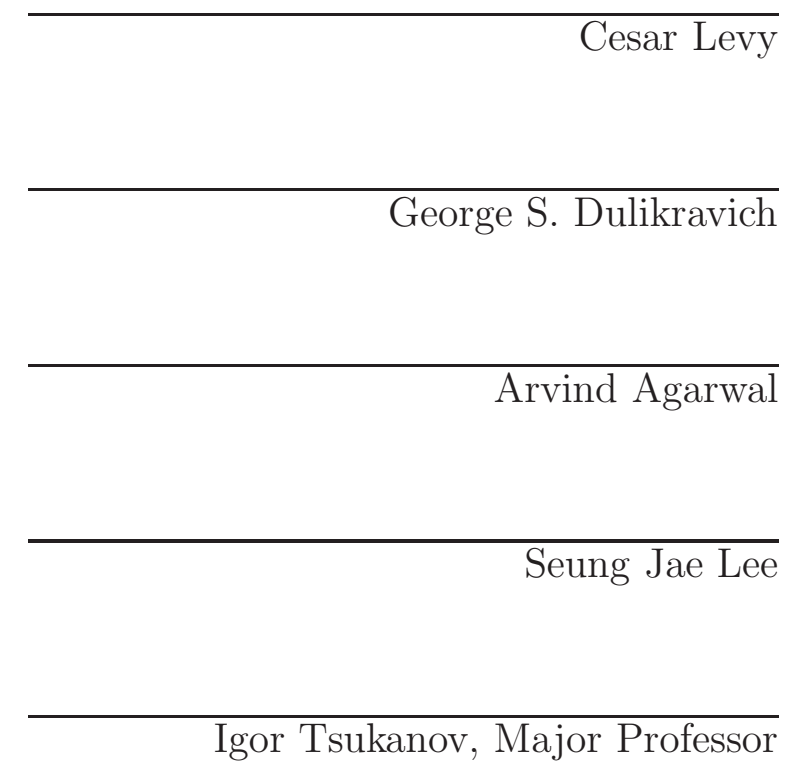

Date of Defense: March 25, 2016

The dissertation of Siavash Rastkar is approved.

Interim Dean Ranu Jung

College of Engineering and Computing

Andrés G. Gil

Vice President for Research and Economic Development and Dean of the University Graduate School

Florida International University, 2016 
(C) Copyright 2016 by Siavash Rastkar

All rights reserved. 


\section{DEDICATION}

To my parents. 


\section{ACKNOWLEDGMENTS}

Thanks, everyone who helped me learn more! 


\title{
ABSTRACT OF THE DISSERTATION \\ CHARACTERIZATION OF HOMOGENIZED MECHANICAL PROPERTIES \\ OF POROUS CERAMIC MATERIALS BASED ON THEIR REALISTIC \\ MICROSTRUCTURE
}

by

\author{
Siavash Rastkar \\ Florida International University, 2016 \\ Miami, Florida \\ Professor Igor Tsukanov, Major Professor
}

The recent advances in the Materials Engineering have led to the development of new materials with customized microstructure in which the properties of its constituents and their geometric distribution have a considerable effect on determination of the macroscopic properties of the substance. Direct inclusion of the material microstructure in the analysis on a macro level is challenging since spatial meshes created for the analysis should have enough resolution to be able to accurately capture the geometry of the microstructure. In most cases this leads to a huge finite element model which requires a substantial amount of computational resources.

To circumvent this limitation a number of homogenization techniques were developed. By considering a small element of the material, referred to as Representative Volume Element (RVE), homogenization methods make it possible to include the effects of a materials microstructure on the overall properties at the macro level. However, complexity of the microstructure geometry and the necessity of satisfying periodic boundary conditions introduce additional difficulties into the analysis procedure.

In this dissertation we propose a hybrid homogenization method that combines Asymptotic homogenization with MeshFree Solution Structures Method (SSM). Our 
approach allows realistic inclusion of complex geometry of the microstructure that can be captured from micrographs or micro CT scans. In addition to unprecedented flexibility in handling complex geometries, this method also provides a completely automatic analysis procedure. Using meshfree solution structures simplifies meshing to creating a simple cartesian grid which only needs to contain the domain. This also eliminates manual modifications which usually needs to be performed on meshes created from image data.

A computational platform is developed in $\mathrm{C}++$ based on meshfree/asymptotic method. In this platform also a novel meshfree solution structure is designed to provide exact satisfaction of periodic boundary conditions for boundary value problems such as homogenization. Performance of the developed platform is tested over 2D and 3D domains against previously published data and/or conventional finite element methods. After getting satisfactory results, homogenized properties are used to compute localized stress and strain distributions over inhomogeneous structures.

Furthermore, effects of geometric features of pores/inclusions on homogenized mechanical properties is investigated and it is demonstrated that the developed platform could provide an automated quantitative analysis tool for studying effects of different design parameters on homogenized properties. 


\section{TABLE OF CONTENTS}

1. INTRODUCTION . . . . . . . . . . . . . . . . . 1

1.1 Problem Description and Importance . . . . . . . . . . . . . . . . . 1

1.2 Literature Review . . . . . . . . . . . . . . . . . . . . . 2

1.3 Objectives and Contributions . . . . . . . . . . . . . 10

1.4 Outline of the Dissertation . . . . . . . . . . . . . . . . . . . . 11

2. THEORY AND MATHEMATICAL FORMULATION . . . . . . . . . . 12

2.1 Homogenization, Formulation and Result Assessment . . . . . . . . . . . 12

2.1.1 Homogenization . . . . . . . . . . . . . . . . . . . . . . . . . . 12

2.1 .2 Stress distribution . . . . . . . . . . . . . . . . . . . . . . 18

2.1.3 Validation of homogenized properties . . . . . . . . . . . . . . . 19

2.2 MeshFree . . . . . . . . . . . . . . . . . . . . . . . . . 23

2.2 .1 Solution Structure Method . . . . . . . . . . . . . . . . . . . . . 23

2.2 .2 Distance field functions . . . . . . . . . . . . . . . . . . . 27

2.2 .3 Automatic differentiation and Integration . . . . . . . . . . . . . 29

2.2 .4 Image Acquisition . . . . . . . . . . . . . . . . . . . . . . 33

3. HOMOGENIZED MECHANICAL PROPERTIES OF INHOMOGENEOUS

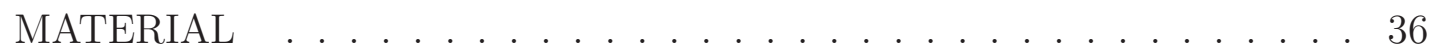

3.1 MeshFree/Asymptotic Platform . . . . . . . . . . . . . . . 36

3.1.1 Solution structure for periodic boundary conditions . . . . . . . . . . . 37

3.1 .2 Software prototype work flow . . . . . . . . . . . . . . . 44

3.2 Numerical Experiments . . . . . . . . . . . . . . . . . . . . . . . . . 47

3.2 .1 Geometries mentioned in literature . . . . . . . . . . . . . . . 47

3.2 .2 Fully anisotropic geometries . . . . . . . . . . . . . . . . 56

3.2 .3 Geometries captured from images . . . . . . . . . . . . . . . . 58

4. STRESS AND STRAIN DISTRIBUTION . . . . . . . . . . 73

4.1 Stress-Strain Localization Methodology . . . . . . . . . . . . . . . . 73

4.2 Numerical Experiments . . . . . . . . . . . . . . . . . . . . . . . . 74

4.2 .1 Carbon/Epoxy composite . . . . . . . . . . . . . . . . 75

4.2 .2 Square RVE with a square void . . . . . . . . . . . . . . . . . . 79

4.2 .3 Spark plasma sintered $\mathrm{TaC} \ldots \ldots$. . . . . . . . . . . 86

5. EFFECTS OF GEOMETRIC FEATURES ON MECHANICAL PROPER-

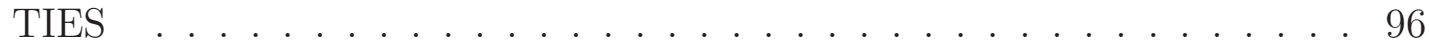

5.1 Effect of volume fraction . . . . . . . . . . . . . . . . . . . . 98

5.2 Effect of pore concentration . . . . . . . . . . . . . . . . 100

5.3 Effect of pore shape . . . . . . . . . . . . . . . 106 
6. HOMOGENIZATION OF THREE DIMENSIONAL DOMAINS . . . . . 108 6.1 Layered Composite . . . . . . . . . . . . . . . . . . . . 109

6.2 Composite Matrix Reinforced with Boron Fiber . . . . . . . . . . . . . . 111

6.3 Cubic Grid-Reinforced Composite with Orthotropic Reinforcement . . 116

7. CONCLUSION . . . . . . . . . . . . . . . . . . . 119

BIBLIOGRAPHY . . . . . . . . . . . . . . . 125

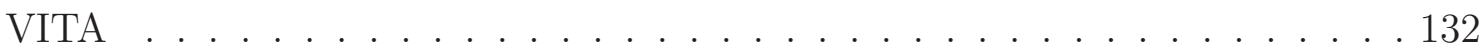




\section{LIST OF TABLES}

TABLE

PAGE

2.1 Solution structures corresponding to different types of boundary conditions for second order partial differential equations. . . . . . . . 26

3.1 Homogenized stiffness matrix of the RVE for perforated plate. . . . . 59

3.2 Homogenized stiffness matrix for the RVE obtained from SEM image of $\mathrm{TaC}$. Values are derived by assuming plane stress and $E=$

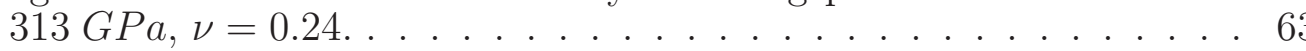

3.3 Homogenized stiffness matrix for the RVE representing AlSi-CNT. Values are derived by assuming plane stress and $E_{\text {matrix }}=74.52 \mathrm{GPa}$, $\nu_{\text {matrix }}=0.33, E_{\text {fiber }}=600 G P a$ and $\nu_{\text {fiber }}=0.27 \ldots$. . . . . . 67

3.4 Homogenized stiffness matrix for the RVE representing Al-10-CNT. Values are derived by assuming plane stress and $E_{\text {matrix }}=74.52 \mathrm{GPa}$, $\nu_{\text {matrix }}=0.33, E_{\text {fiber }}=600 G P a$ and $\nu_{\text {fiber }}=0.27 \ldots$. . . . . . 70

6.1 Mechanical properties of matrix and reinforcement for the layered composite. . . . . . . . . . . . . . . . . . . 110

6.2 Homogenized stiffness matrix of the layered composite RVE. . . . . . 112

6.3 Mechanical properties of matrix and fiber. . . . . . . . . . . . 113

6.4 Homogenized stiffness matrix of the composite with fiber reinforcement.114

6.5 Mechanical properties of matrix and fiber. . . . . . . . . . . 117

6.6 Homogenized stiffness matrix of the composite with reinforcement grid. . . . . . . . . . . . . . . . . . . . . 118 


\section{LIST OF FIGURES}

FIGURE

1.1 Schematic diagram showing the relation between processing, structure, properties and behaviour of an advanced material. Red boxes represent the areas which this dissertation investigates. . . . . . . . 2

1.2 The distance field functions constructed from the boundary representation of state of Wisconsin. . . . . . . . . . . . . . 7

1.3 Implementation of the 'Automatic Differentiation' and 'Adaptive Integration' in meshfree analysing system. . . . . . . . . . . . . . 9

2.1 Schematic representation of global and local coordinate systems. . . 13

2.2 (a) Domain $\Omega$ subjected to body force $\mathbf{f}$, traction force $\mathbf{t}$ and prescribed displacement boundary condition; (b) Corresponding RVE in local coordinate system. . . . . . . . . . . . . . . . . . . 14

2.3 (a) The native geometry, a right angled triangle, broken down into intersection of three primitive half spaces; (1) under line 1, (2) left side of line 2 and (3) above line 3. (b) Inequalities for $\omega_{1}, \omega_{2}$ and $\omega_{3}$ plotted as functions of $x$ and $y$ and the resulting $R_{0}$-conjunction $\omega=\omega_{1} \wedge_{0} \omega_{2} \wedge_{0} \omega_{3}$. The $\omega$ function has zero values and non-zero gradients on the boundaries and it is differentiable in the whole domain. 29

2.4 (a) Cartesian coordinates used for boundary cells with at least one face fully contained in the domain (b) Cylindrical coordinates for boundary cells with at least on edge in the domain (c) Spherical coordinates for boundary cells with at least one vertex in the domain. 33

3.1 Construction of approximate distance fields using $R$-functions, (a) The native geometry is divided into five half-spaces, (b) Inequalities for $\omega_{1}$ to $\omega_{5}$ plotted as functions of $x$ and $y$ and the resulting $R_{0^{-}}$ conjunction $\omega$ has zero values and non-zero gradients on the boundaries and it is differentiable in the whole domain. . . . . . . . . . . 40

3.2 Basis functions created over a Cartesian grid on the domain. . . . . . 41

3.3 Enrichment functions created in pairs via eq. (3.15) for $n^{\prime}=6$. . . 42

3.4 Schematic for a tuple sequence structure. . . . . . . . . . . . . . . 43

3.5 (a) Nonconforming uniform grid over the domain, (b) Distribution of Gauss integration points in the uniform grid cells, (c) Nonconforming grid with refined boundary cells, (d) Distribution of Gauss integration points in the refined grid cells. . . . . . . . . . . . . . 44

3.6 Block diagram for Meshfree/Asymptotic homogenization platform. . 46 
3.7 Control parameters accessible by user in the Meshfree/Asymptotic homogenization platform. . . . . . . . . . . . . . 47

3.8 RVE for a composite with periodic circular inclusions $\left(V_{f}=0.25\right) \ldots 48$

3.9 Geometry modeling (a) Distance field functions for epoxy; (b) Distance field functions for carbon fiber; (c) Nonconforming grid over the domain; (d) Gauss integration points distribution. . . . . . . . . 49

3.10 Basis $\left\{\chi_{m}^{k l}\right\}$ functions for RVE with circular inclusions. . . . . . . . 50

3.11 Homogenized (a) Young's and (b) Shear modulus for a square RVE with a circular fiber computed with MeshFree method and compared with finite elements results and Hashin-Shtrikman upper and lower bounds. . . . . . . . . . . . . . . . . . 50

3.12 RVE for a square domain with a square void $\left(V_{f}=0.70\right) . \ldots \ldots 51$

3.13 Construction of approximate distance field functions for a RVE with a square void using $R_{0}$-conjunction. . . . . . . . . . . 51

3.14 (a) Nonconforming grid over the RVE; (b) Distribution of Gauss integration points. ................... 52

3.15 Basis $\left\{\chi_{m}^{k l}\right\}$ functions for RVE with a square void and $v_{f}=0.70 . \quad$. 52

3.16 Elements of homogenized elasticity tensor (a) $E_{1111}^{H}$ (b) $E_{1212}^{H}$ as functions of material volume fraction, computed with meshfree and compared to finite element results. . . . . . . . . . . . . . . 53

3.17 RVE for a masonry block with mortar thickness $t=20 \mathrm{~mm} . \ldots . .54$

3.18 Geometry modeling for a masonry block with $t=20 \mathrm{~mm}$ (a) Distance field functions for brick; (b) Distance field functions for mortar; (c) Nonconforming grid over the domain; (d) Gauss integration points distribution. . . . . . . . . . . . . . 55

3.19 Basis $\left\{\chi_{m}^{k l}\right\}$ functions for a masonry RVE with $t=20 \mathrm{~mm} . \ldots . .55$

3.20 Meshfree/asymptotic results compared with eigenstrain method. . . . 56

3.21 Geometry modeling for a perforated plate (a) RVE; (b) Distance field functions; (c) Nonconforming grid over the domain; (d) Gauss integration points distribution. . . . . . . . . . . . 57

3.22 Basis $\left\{\chi_{m}^{k l}\right\}$ functions for a perforated plate. . . . . . . . . 58

3.23 COMSOL model of the RVE for perforated plate, KUBC boundary conditions and elements of homogenized elasticity tensor corresponding to each set of boundary conditions. . . . . . . . . . . . . 59 
3.24 Geometry modeling of TaC (a) Grayscale image of the microstructure (b) Binary representation of the geometry (c) Sampled Euclidean distances $(\mathrm{d})$ Distance field functions . . . . . . . . . . . . . 60

3.25 3D presentation of distance field functions constructed over the SEM image of spark plasma sintered TaC . . . . . . . . . . . . . . . 61

3.26 Geometry modeling of TaC (a) Nonconforming grid over the domain; (b) Gauss integration points distribution. . . . . . . . . . . . . . 62

3.27 Basis $\left\{\chi_{m}^{k l}\right\}$ functions for the SEM image of spark plasma sintered TaC . . . . . . . . . . . . . . . 62

3.28 OOF2 software setup with a microstructure image imported as source of the geometry. . . . . . . . . . . . . . . . . 64 64

3.29 (a) The initial mesh on the TaC microstructure image (b) After refining the mesh in vicinity of porosities (c) After snapping the nodes close to internal boundaries to the boundaries and smoothing the elements (d) After performing steps $b$ and $c$ for elements which are still consisted of two different materials and rationalizing and fixing illegal elements; Elements representing the porous area are colored to show the captured geometry by OOF2. Marked areas with same numbers show the regions which mesh still needs modifications to have matching node distribution on opposing edges in order to perform node pairing to satisfy periodic boundary conditions. . . .

3.30 Geometry modeling of AlSi-CNT (a) SEM image of the microstructure (b) Binary representation of the geometry (c) Sampled Euclidean distances. . . . . . . . . . . . . . . . . 66

3.31 Geometry modeling of AlSi-CNT (a) Distance field representation of the matrix (b) Distance field representation of the fibers (c) Nonconforming grid for both phases (d) Gauss integration points distribution. 66

3.32 Basis $\left\{\chi_{m}^{k l}\right\}$ functions for the SEM image of AlSi-CNT. . . . . . . 67

3.33 The final finite element mesh for AlSi-CNT, created with OOF2 and manually modified. Areas which still needs modifications for performing node pairing are marked. . . . . . . . . . . . . . . . . .

3.34 Geometry modeling of Al-10-CNT (a) Grayscale image of the microstructure (b) Binary representation of the geometry (c) Sampled Euclidean distances. . . . . . . . . . . . . . . . . .

3.35 Geometry modeling of Al-10-CNT (a) Distance field representation of the matrix (b) Distance field representation of the fibers (c) Nonconforming grid for both phases (d) Gauss integration points distribution. . . . . . . . . . . . . . . 69

3.36 Basis $\left\{\chi_{m}^{k l}\right\}$ functions for the SEM image of Al-10-CNT. . . . . . . 70 
3.37 The final finite element mesh created over Al-10-CNT domain by OOF2 and manually modified. Areas which still needs modifications for performing node pairing are marked. . . . . . . . . . . . . . . 71

4.1 Geometry modeling (a) RVE; (b) Global domain with a $2 \times 2$ grid and boundary conditions ; (c) Global domain with a $10 \times 10$ grid and boundary conditions (d) Homogenized problem. . . . . . . . . 76

$4.2 L_{2}$-norms calculated for strains and stresses with different values of $\epsilon .77$

4.3 Error distribution for displacement over the global domains . . . . . 80

4.4 Error distribution for strains over the global domains . . . . . . . . . 81

4.5 Error distribution for strains over the global domains . . . . . . . . 82

4.6 Geometry effects for the global domain with $\epsilon=0.1$ (a) $u_{1}^{1}(\boldsymbol{x}, \boldsymbol{y})$; (b) $u_{2}^{1}(\boldsymbol{x}, \boldsymbol{y}) . \ldots \ldots \ldots 3$

4.7 Total displacements calculated with full geometric model and homogenized model. . . . . . . . . . . . . . 83

4.8 Strains calculated with full geometric model and homogenized model. 84

4.9 Stresses calculated with full geometric model and homogenized model. 84

4.10 Geometry modeling (a) RVE; (b) Global domain with a $1 \times 4$ grid and boundary conditions ; (c) Global domain with a $5 \times 20$ grid and boundary conditions(d) Homogenized problem. . . . . . . . . . . 85

$4.11 L_{2}$-norms calculated for strains and stresses with different values of $\epsilon .85$

4.12 Error distribution for displacement over the global domains . . . . . 86

4.13 Error distribution for strains over the global domains . . . . . . . . . 87

4.14 Error distribution for stresses over the global domains . . . . . . . . 87

4.15 Total displacements calculated with full geometric model and homogenized model for a global domain constructed from square RVEs

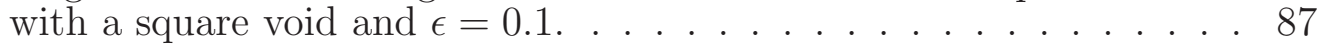

4.16 Strains and corresponding error distributions for a global domain constructed from square RVEs with a square void and $\epsilon=0.1$. . . . 88

4.17 Stresses and corresponding error distributions for a global domain constructed from square RVEs with a square void and $\epsilon=0.1$. . . . 88

4.18 Geometry modeling (a) RVE; (b) Global domain with a $2 \times 2$ grid and boundary conditions ; (c) Global domain with a $10 \times 10$ grid and boundary conditions $(\mathrm{d})$ Homogenized problem. . . . . . . . . . 89 
$4.19 L_{2}$-norms calculated for strains and stresses with different values of $\epsilon .89$

4.20 Error distribution for displacement over the global domains . . . . . 91

4.21 Error distribution for strains over the global domains . . . . . . . . . 92

4.22 Error distribution for stresses over the global domains . . . . . . . . 93

4.23 Strains and corresponding error distributions for a global domain constructed from square RVEs with a square void and $\epsilon=0.1$. . . . 94

4.24 Stresses and corresponding error distributions for a global domain constructed from square RVEs with a square void and $\epsilon=0.1$. . . . 95

5.1 A unit length RVE with random distribution of circular porosities inside. Pores are distributed in a rectangular $0.7 \times 0.3$ area centered in the RVE with edges (a) parallel to $x$ and $y$ axis, (b) with a $45^{\circ}$

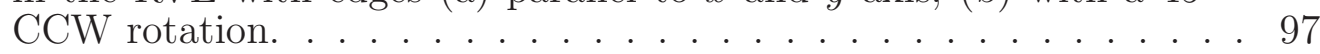

5.2 Elasticity tensor members as a function of rotation angle, (a) $E_{1111}$ and $E_{2222}$, (b) $E_{1212}$ and $E_{1122}$, (c) $E_{1112}$ and $E_{1211} \ldots \ldots$. . . . 97

5.3 A unit length RVE with random distribution of different volume fractions of fibers. (a) $5 \%$, (b) $8 \%$, (c) $12 \%$, (d) $15 \%$, (e) $18 \%$, (f)

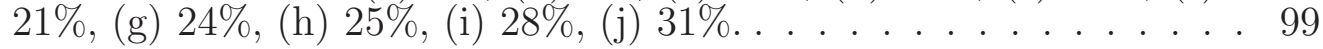

5.4 Elasticity tensor members as a function of fiber volume fraction, (a) $E_{1111}$ and $E_{2222}$, (b) $E_{1212}$ and $E_{1122} \ldots \ldots \ldots$. . . . . . . 99

5.5 A unit length RVE with random distribution of circular porosities inside. Pores are distributed in a rectangular (a) $0.7 \times 0.3$, (b) $0.7 \times$ 0.4, (c) $0.7 \times 0.5$, (d) $0.7 \times 0.6$ area. . . . . . . . . . . 100

5.6 Elasticity tensor members as a function of rotation angle for different areas of pore distribution. (a) $E_{1111}$, (b) $E_{2222}$, (c) $E_{1212} \ldots 101$

5.7 Elasticity tensor members as a function of moment of inertia for different areas of pore distribution. . . . . . . . . . . . . 102

5.8 A unit length RVE with random distribution of circular porosities inside. Pores are distributed in 4 rectangular $0.35 \times 0.15$ areas with edges (a) parallel to $x$ and $y$ axis, (b) with a $45^{\circ}$ CCW rotation. . . . 103

5.9 Elasticity tensor members as a function of rotation angle for pores distributed in a single rectangle and four separated ones. (a) $E_{1111}$, (b) $E_{2222}$, (c) $E_{1212}$, (d) $E_{1122}$, (e) $E_{1112}$, (c) $E_{2212} \ldots \ldots$. . . . . . 103

5.10 A unit length RVE with random distribution of a circular inclusions as reinforcement. Inclusions are distributed in a rectangular (a) $0.7 \times$ 0.3 , (b) $0.7 \times 0.4$, (c) $0.7 \times 0.5$, (d) $0.7 \times 0.6$ area. . . . . . . . . . 104 
5.11 Elasticity tensor members of a RVE with two different materials as a function of rotation angle for different areas of pore distribution.

(a) $E_{1111}$, (b) $E_{2222}$, (c) $E_{1212} \ldots \ldots$. . . . . . . . . . 104

5.12 Elasticity tensor members as a function of moment of inertia for different areas of pore distribution. . . . . . . . . . . . . 105

5.13 Elasticity tensor members as a function of rotation angle for inclusions distributed in a single rectangle and four separated ones. (a) $E_{1111}$, (b) $E_{2222}$, (c) $E_{1212}$, (d) $E_{1122}$, (e) $E_{1112}$, (c) $E_{2212}$. . . . . . 105

5.14 A unit length RVE with random distribution of pores inside. Pores are distributed in a $0.7 \times 0.3$ rectangular area and modeled with (a) ellipses, (b) Squares. . . . . . . . . . . . . . . . 106

5.15 Elasticity tensor members as a function of rotation angle for pores modeled with circles, ellipses and squares. (a) $E_{1111}$, (b) $E_{2222}$, (c) $E_{1212}$, (d) $E_{1122}$, (e) $E_{1112}$, (c) $E_{2212}$................. 107

5.16 Elasticity tensor members as a function of rotation angle for inclusions modeled with circles, ellipses and squares. (a) $E_{1111}$, (b) $E_{2222}$, (c) $E_{1212}$, (d) $E_{1122}$, (e) $E_{1112}$, (c) $E_{2212}$. . . . . . . . . . 107

6.1 RVE geometry for a composite structure with layer reinforcement. . . 110

6.2 Distance field functions for matrix and reinforcement layer and Gauss integration point distribution in a section of domain. . . . . . . . 111

$6.3 \chi_{1}^{k l}$ functions for layered composite. . . . . . . . . . . . . . . 112

6.4 RVE geometry for a composite structure with fiber reinforcement,

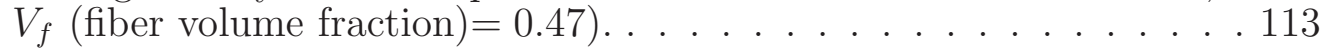

6.5 Distance field functions for matrix and reinforcement fiber and Gauss integration point distribution in a section of domain. . . . . . . . . . 114

$6.6 \chi_{m}^{k l}$ functions for composite with fiber reinforcement. . . . . . . . . . 115

6.7 RVE geometry for a composite structure with reinforcement grid, $V_{f}$

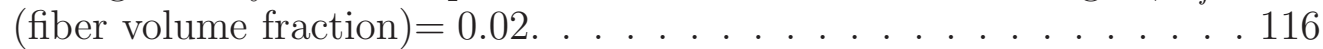

6.8 Distance field functions for matrix and reinforcement grid and Gauss integration point distribution in a section of domain. . . . . . . . 117

7.1 MeshFree/Asymptotic Platform . . . . . . . . . . . . . . . . 120

7.2 (a) RVE for Al-10-CNT, (b) Solution time comparison for conventional finite elements and meshfree solution structures. . . . . . . . . 122 


\section{CHAPTER 1 \\ INTRODUCTION}

\subsection{Problem Description and Importance}

Advances made in different manufacturing fields were always linked to technological breakthroughs in robotics, information technology and high performance computing, but nothing can affect how things are made and what they are capable of more than the materials used by manufacturers. Significant advances in structural material technologies have been made in the past 50 years and new materials such as ceramics and composites have shown superior properties in comparison to traditional ones such as steel and aluminium. Until the 1950s, the most important ceramic materials were pottery, bricks, tiles and cement. After a few decades, in the early 90's the value of components produced with advanced materials was almost $\$ 2$ billion per year and by 2000 this number was doubled [10, 32].

In general materials such as composites and ceramics can be described as materials made from dissimilar constituents formed into inhomogeneous structures with methodical or random geometric distribution. One of the difficulties in the widespread application of advanced materials is understanding the underlying mechanisms behind the processing-structure-property relationship of them [32]. In other words, how can selection of constituents and processing route affect the microstructure of material and how to predict the properties of a certain microstructure. Currently these problems are being investigated quite specifically and research programs are focusing on understanding the mentioned underlying relations. The physical properties of these materials are highly dependent on three main factors: (1) the properties of base materials used to make the final product; (2) spatial distribution and shape of the inhomogeneities ; (3) the relative density of the product [49]. 


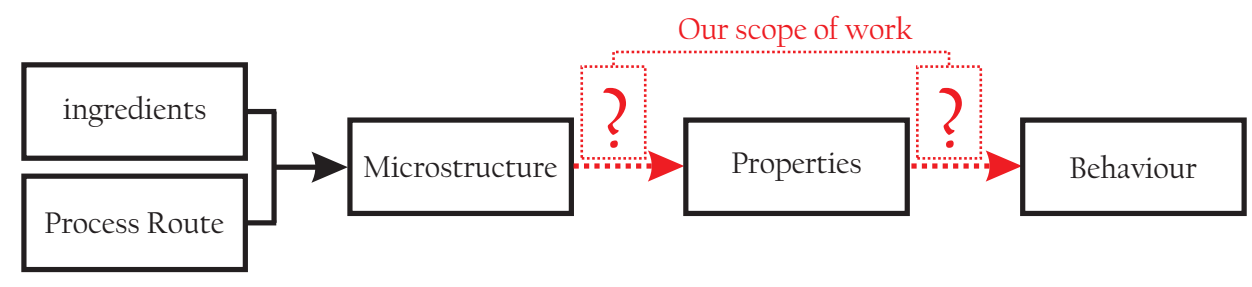

Figure 1.1: Schematic diagram showing the relation between processing, structure, properties and behaviour of an advanced material. Red boxes represent the areas which this dissertation investigates.

Therefore one of the main challenges in creating new materials with desired properties is controlling inhomogeneity (or more specifically pores) sizes and distributions and studies have been conducted to investigates methods for controlling the size of porosities during the manufacturing process $[1,61]$ or how to model porous materials and investigate the effect of pore sizes and topology on material behavior [14]. Here in this dissertation our work is mainly concentrated on developing a computational platform to improve our understanding of the links between material microstructure architecture, its properties and mechanical behaviour of the material (Fig. 1.1).

\subsection{Literature Review}

In many engineering cases despite the fact that the materials involved are not homogenous we are still interested in having an overall understanding of their behavior. One of the most common techniques for doing this is homogenization, which allows us to calculate effective material characteristics based on properties of particular components and their volume fraction in the structure. Considering the vast ap-

plications of ceramic and composite materials and the undeniable importance of understanding their mechanical behavior, here in this work we are going to focus on investigating their homogenized mechanical properties based on a realistic micro structure model. 
Attempts to find out the properties of composite materials started about 100 years ago and at that time it was as simple as rule of mixtures. This general rule is a weighted mean used to calculate various properties of composite materials by predicting an upper and a lower bound for material properties [51] However this simple model could not provide a precise tool but it was the base for developing more advanced analytical methods to predict material properties. In early 60s Hashin and Shtrikman were able to provide a variational approach for predicting bounds on the effective properties. Although the method proposed by them was much more accurate than simple rule of mixtures but it was limited to two phase materials and it depended only on the relative volumes and did not take the geometry into consideration $[18,19]$. To overcome this weakness, a few years later Hill proposed a self consistent method for composite materials and he was able to calculate the macroscopic elastic moduli of two-phase composites with ellipsoidal inclusions [27, 26]. Later in 1973 Mori and Tanaka discussed a method for calculating the average internal stress in the matrix of composite materials and they showed that the actual stress in the matrix is the average stress plus the local fluctuations [44]. This method was modified in 1987 by Benveniste for computation of the effective properties of composites [5].

Although different analytical methods have been proposed for homogenization, but all of them are often limited to specific cases. On the other hand since numerical methods have more flexibility in handling complex problems they seem to be a more appropriate tool to deal with homogenization problem. Most of classic numerical homogenization techniques follow similar steps. In these methods to reach to an understanding of material's general behavior, instead of studying the whole structure a microstructural representative volume element (RVE) is defined as the repetitive structural pattern. Periodic boundary conditions are applied on opposing faces 
of the mentioned element and governing equations of the problem are solved in the RVE. Then an attempt is made to bridge between micro and macro-scale and predict the global results.

Reliability and efficiency of multi-scale methods has been investigated by different groups. For instance a RVE based homogenization scheme for a periodic porous composite is compared to direct analysis and the error is investigated as a function of the ratio of the RVE size to global domain dimensions [28]. Also adaptive methods for hierarchical modeling of heterogenous structures are introduced where modeling errors created by replacing the actual structure with a representative element are investigated [72] and required RVE scale in order to meet a preset error was determined [46].

So far various numerical methods have been used to tackle the multi-scale equations of a homogenization problem and among all these methods Finite Element Analysis is the most popular one, due to its flexibility in handling different type of problems, being computer programming friendly and the availability of commercial FE software $[8,15]$. Extensive studies have been carried out about FE homogenization techniques. Their computational issues and applications were overviewed and a relationship between macro and micro-scale properties were developed [8, 41]. Mathematical expansions for modeling physical phenomena on inhomogeneous materials with periodic microstructure and also the explicit mathematical equations which describes the local stress and strain fields associated with a given global domain were derived [50]. Performance of such formulations were tested on composite structures and effect of reinforcement volume fraction and their geometry and distribution in the matrix on overall material properties were studied [47].

Behavior of different type of elements like thin structure shell and beam elements are studied in thermal, mechanical and coupled problems and they have been ap- 
plied in solving in plane loading problems for 3D beams with periodic structure [7]. Derivation of homogenization equations are studied in details for computation of effective constitutive parameters, analytical and finite element formulation of a group of simple geometries such as rank laminated composites are compared with each other and results are used for structural topology optimization to obtain desired material properties [20, 21, 22]

In many cases where finding the effective property of a non-homogenous material was the goal, computational homogenization methods have proven to be a very powerful tool. The numerical investigation of stress and strain of orthotropic composite materials has yield to determining their average (effective) stress and strain energy [70] and also combining asymptotic homogenization and finite element is used to depict the evaluation of microstructural stresses and strains [16]

Similar studies have been performed on reinforced composites to find out their effective shear modulus and effect of volume fraction of particles on it [31]. Thermal properties of two-phase materials were investigated by applying a FE homogenization scheme and as the result effective thermal expansion coefficient was calculated [43], same process has been implemented on non-homogenous materials to find out their effective Youngs modulus [42].

Although finite element, as the most popular computational homogenization technique, is a very robust and developed method but it still carries some weaknesses. Generally in any finite element method the domain of interest is divided into very small elements which their sizes are determined by size of the smallest features in the domain, but due to computational costs and concerns there is a limit on how small these meshes can be. As a result, the geometrical complexity that can be captured by them is limited. Also in cases where geometry is presented by detailed pictures of the microstructure there are very few software packages which are capable 
of performing image-based mesh generation. And not only they are considerably expensive but also substantial amount of manual work is required for fine tuning the meshes. furthermore, finite element is based on continuity in elements and modeling frequent breakages of material which often happens in a homogenization problem can be another source of problems for a finite element user.

Most importantly in any RVE-based homogenization method, due to the periodic nature of the structure, it is of great importance to be able to apply periodic boundary conditions on the faces of selected RVE. The most common approach for mesh-based finite element methods to impose periodic boundary conditions on opposing sides of a RVE involves pairing nodes on opposite faces. But in a general setting the similarity of mesh distribution on paired boundaries of RVE cannot always be guaranteed, especially in cases where the geometry of pores inside the RVE requires a non-periodic mesh or pores intersect with RVE sides. Both these cases are very common when the RVE model is cpatured from a real micro structure image and contains a non-periodic mesh which can make the node pairing process very difficult to apply $[45,68]$.

To overcome drawbacks of conventional finite element, which mainly present themselves during the mesh generation stage, meshfree or meshless methods have been developed. Over the past 15 years, meshfree techniques have presented themselves as a powerful numerical tool for dealing with different types of engineering problems. The basic idea behind modeling a certain geometry without using meshes was proposed by Rvachev in 1963, he presented functions called $R$-functions which were used to model domain geometry and form a solution structure in terms of a functional expression which captures the native geometry and satisfies the given boundary conditions $[53,54]$.

In meshfree method instead of using a geometry conforming mesh to represent 

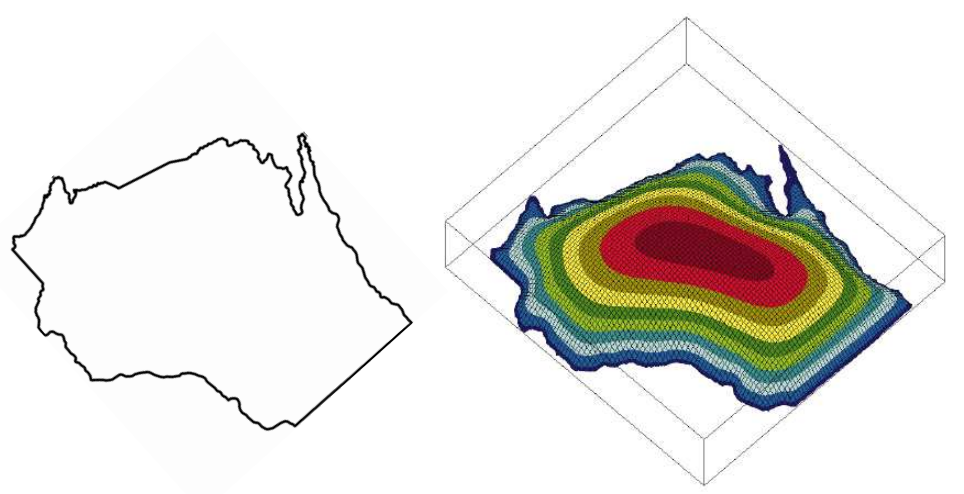

Figure 1.2: The distance field functions constructed from the boundary representation of state of Wisconsin [59].

the model, a group of implicit functions are utilized to describe the domain. These functions are designed in such way that they have zero values on the boundary, positive in the domain and negative outside. A sample geometry modeled via distance field functions is shown in Fig. 1.2. In 1999 Shapiro and Tsukanov started a detailed study on theory of $R$-functions and they explored different methods for generation of implicit functions (also known as distance field functions). Based on whether the native domain is represented in the form of a CSG (Constructive Solid Geometry) or a boundary representation, different methods could be implemented to generate the proper distance field functions [59].

Having the distance field representation of a domain makes it possible to create a solution structure for the boundary value problem imposed on it. The resulting Solution Structure Method (SSM) contains necessary terms to capture the geometric effect and also can provide exact satisfaction of boundary conditions and it is in fact meshfree, which means the spatial discretization no longer needs to conform the geometry of the domain [56]. It was also shown that by a proper choice of $R$-function the SSM can converge to the exact solution of the problem. Efficiency of the method was tested in a sample non-steady heat transfer problem with non-homogenous 
mixed boundary conditions. In similar works it was also shown that $R$-functions can be used in automated modeling of problems with time-varying geometries $[59,56]$.

In addition to the development of SSM, researches were carried out to investigate the properties of functions involved in it. An algorithm was proposed for direct numerical computation of partial derivative of mentioned functions. It was shown that by application of generalized Leibnitz rules, constant time access can be provided to the partial derivatives which accelerates the automatic differentiation process [63]. Also a novel technique was formulated for geometrically adaptive numerical integration. Numerical integration over solid domains usually requires adoption to the solid boundaries, but it was demonstrated that by combining a system similar to marching cubes with suitable parameterizations of the boundary cell geometry a greater accuracy with fewer integration point can be provided [39].

After investigating the mathematics behind SSM and the advantages that $R$ functions can provide, solution structures were studied more systematically and they were classified for different types of boundary conditions. The possibility of automatic construction of them were evaluated and it was shown that how distance field functions in conjunction with transfinite interpolation can be used toward exact satisfaction of boundary conditions. Previously developed automatic differentiation and integration toolboxes were used in run time and over non-meshed domains to deal with case studies such as heat transfer with mixed Neumann and Dirichlet boundary conditions, plate vibration, stress in a cantilever beam and incompressible fluid dynamic [64, 55]. Fig. 1.3 shows how the automatic differentiation and integration toolboxes can be implemented in a meshfree analysis engine.

Considering the ability and flexibility of meshfree methods in capturing complicated geometries, they were also applied in modeling non-homogenous materials. It was shown that using generalized Taylor series expansions to describe all dis- 


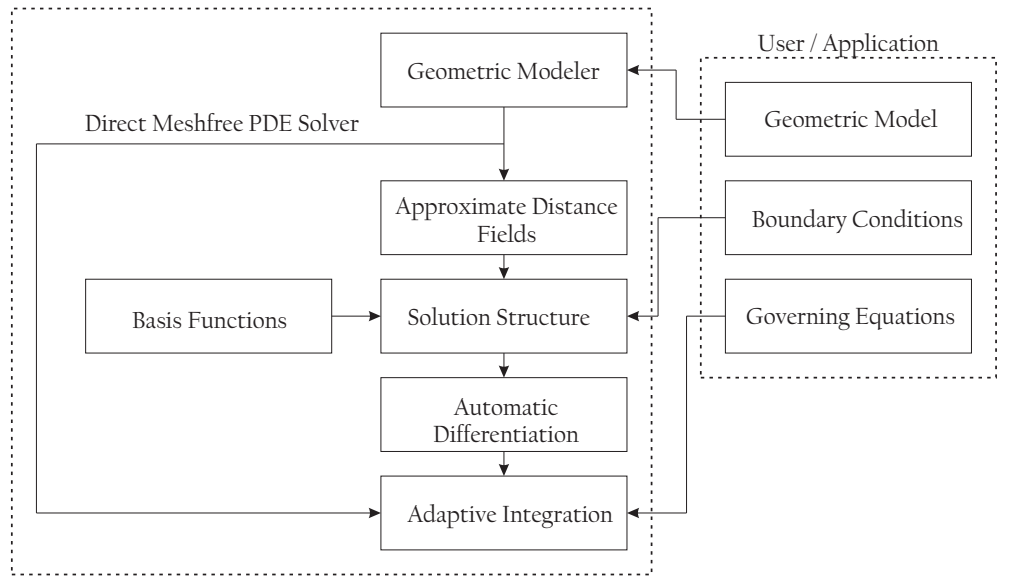

Figure 1.3: Implementation of the 'Automatic Differentiation' and 'Adaptive Integration' in meshfree analysing system [64].

tributions of physical properties and also employing distance fields to boundaries and material features can provide a powerful tool to represent variations of material properties and also satisfy prescribed boundary conditions [65].

This capability was later used as a base for developing a software platform called Scan and Solve for simulation of physical fields [11]. In the mentioned platform physical domains can be captured by surface or volumetric scanning technologies, constructive solid geometry, polygonized model and even appropriately interpreted point cloud. Since Scan and Solve is a completely meshfree paradigm it can provide the means for automated field simulation and bypassing geometric and topological meshing problems $[13,12]$.

Based on the survey conducted on literature about trends in homogenization field it can be noticed that although finite element methods are the most widely used tools in homogenization problems, there still exist challenges in applying them. In most homogenization problems geometric models are provided by micrograph images and due to the mathematical approach used for almost all homogenization techniques, they all involve solving a boundary value problem with periodic boundary conditions. 
Solution to such problems involves converting the captured image into a model understandable by meshing software packages. Meshing has to be done in a manner which provides possibility of node-pairing on opposing faces, but in a general setting the similarity of mesh distribution on paired boundaries of RVE cannot always be guaranteed, especially in cases where the geometry of inhomogeneities inside the RVE requires a non-periodic mesh or they intersect with RVE sides.

Considering the nature of meshfree methods, it seems taking advantage of them will allow us to tackle homogenization problems in a more efficient way by complete elimination of meshing process. This way all the manual work required to fine tune the meshes and make them suitable for a problem with periodic boundary conditions will vanish and numeric simulation of homogenization problems will be more automatized and eventually will lead to cheaper and faster simulations.

\subsection{Objectives and Contributions}

The main idea behind this research is that the overall mechanical properties of a porous media are dependent on the geometry and distribution of porosities in micro structure. In addition to material properties; geometry, volume fraction and location of pores would affect not only the overall mechanical properties, but also will have an impact on stress/strain distribution in the material.

In this dissertation we developed a computational platform to:

- Acquire graphical representation of material structure via 2D microgrpahs and convert them into approximate distance field representations.

- Assemble a proper meshfree solution structure for boundary value problems with periodic boundary conditions by using enrichment basis functions. 
- Combine a meshfree solution structure with asymptotic homogenization to predict overall mechanical properties of multi-phase inhomogeneous structures.

- Use the homogenized properties in conjunction with the meshfree method to find the stress/strain distribution in the domain.

- Study the effect of geometric distribution of inhomogeneities on the overall material properties.

\subsection{Outline of the Dissertation}

The rest of the dissertation is organized as follows: Chapter 2 describes the theory of asymptotic homogenization and how physical fields can be expressed in two micro and macro domains. Also concept and mathematics behind meshfree solution structure is discussed in detail. Chapter 3 explains the hybrid meshfree/asymptotic homogenization method and related formulations, it also contains numerical examples used to validate the performance of our platform. In Chapter 4 homogenized properties are used to investigate stress/strain distribution in an inhomogeneous domain and necessary numerical experiments are presented. Chapter 5 contains the study on effect of geometry on overall material mechanical properties. Homogenization of three dimensional domains is studied in Chapter 6 and discussions and conclusions are presented in Chapter . 


\section{CHAPTER 2}

\section{THEORY AND MATHEMATICAL FORMULATION}

In this chapter the mathematical background for theories and methods used in developing our computational platform is explained in details. As mentioned in

Chapter 1, the proposed platform is formed by combining asymptotic homogenization and meshfree method. Section 2.1 describes the formulation required for calculation of overall properties of inhomogeneous materials, then investigates prediction of stress field distributions and also introduces alternative methods for evaluation of effective properties calculated via homogenization methods.

Mathematical theories involved in meshfree method, such as solution structures for boundary value problems with different types of boundary conditions, distance field functions and automatic differentiation and integration are explained in Section 2.2 .

\subsection{Homogenization, Formulation and Result Assessment}

\subsubsection{Homogenization}

Asymptotic method, as part of homogenization theories, was developed from studying partial differential equations containing rapidly varying coefficients and then it was formed into a powerful tool for finding overall properties of inhomogeneous materials. This method is capable of analyzing inhomogeneous materials with high contrast in the properties of their constituents and in addition to obtaining effective material properties it also solves the full structural problem at micro level $[15,34]$.

It worth mentioning although here we are using asymptotic method, but the developed platform is not limited to that and can be used to empower any other numerical homogenization technique. 


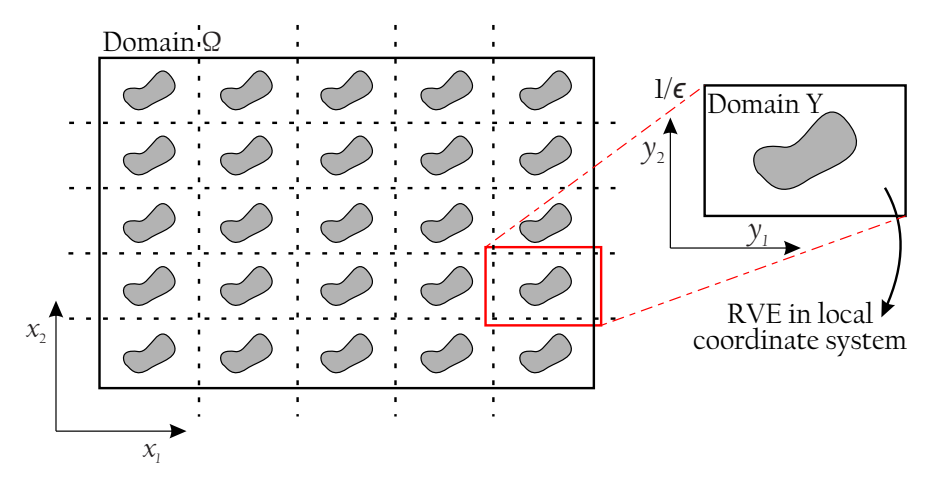

Figure 2.1: Schematic representation of global and local coordinate systems.

As shown in Fig. 2.1, an inhomogeneous material with a periodic microstructure can be represented by domain $\Omega$ in the global coordinate system $\boldsymbol{x}$ and a spatially periodic RVE associated with domain $Y$ in local coordinate system $\boldsymbol{y}$. The ratio of macro and micro coordinate systems is define by $\epsilon$, where $\epsilon<<1$ and is defined in eq. (2.1). For derivation of asymptotic formulation two important assumptions are made [28]:

- Fields vary on multiple spatial scales due to the existence of microstructure.

- The microstructure is spatially periodic.

In asymptotic method if any physical property $(\boldsymbol{\Psi})$ of material can be related to two distinct macro-scale $(\Omega)$ and micro-scale $(Y)$ domains then an expansion in form of eq. (2.2) can be written with respect to $\epsilon$ [20].

$$
\begin{gathered}
\boldsymbol{y}=\frac{\boldsymbol{x}}{\epsilon} \\
\boldsymbol{\Psi}^{\epsilon}(\boldsymbol{x})=\boldsymbol{\Psi}^{0}(\boldsymbol{x}, \boldsymbol{y})+\epsilon \boldsymbol{\Psi}^{1}(\boldsymbol{x}, \boldsymbol{y})+\epsilon^{2} \boldsymbol{\Psi}^{2}(\boldsymbol{x}, \boldsymbol{y})+\ldots
\end{gathered}
$$

Where $\epsilon \rightarrow 0, \epsilon$ superscript shows the periodicity of a given variable on the global coordinate system and $\boldsymbol{\Psi}^{i}$ function are defined in the domain $\Omega$ and they are $Y$-periodic. To evaluate effective properties of inhomogeneous materials it is 


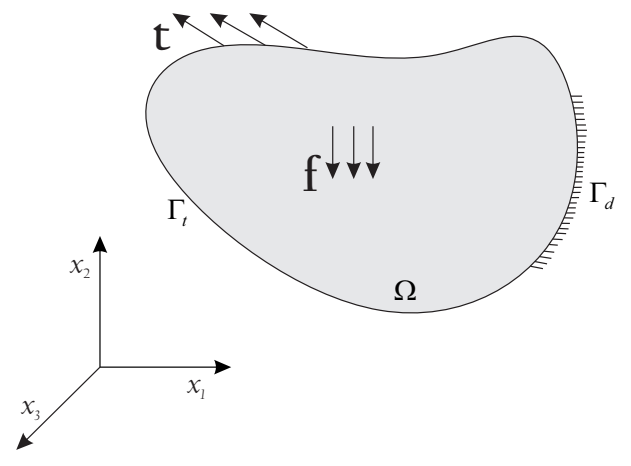

(a)

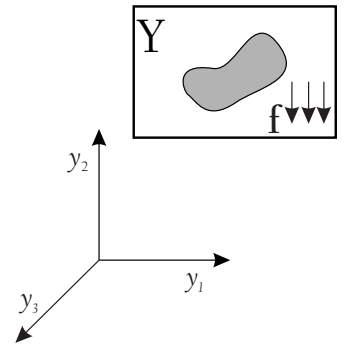

(b)

Figure 2.2: (a) Domain $\Omega$ subjected to body force $\mathbf{f}$, traction force $\mathbf{t}$ and prescribed displacement boundary condition; (b) Corresponding RVE in local coordinate system.

crucial to re-derive governing and constitutive equations in such way that the effect of microstructural inhomogeneities is taken into consideration.

To do so, let's start with a domain $\Omega$ in global coordinate subjected to body force $\mathbf{f}$ and traction force $\mathbf{t}$ on the boundary $\Gamma_{t}$ and prescribed displacement on $\Gamma_{d}$. Mentioned domain has a spatially periodic structure and is made from RVEs which are defined in local coordinate system as shown in Fig. 2.2.

If we assume that the constitutive equation and strain-displacement relation for domain $\Omega$ are defined as eqs. (2.3) and (2.4) and $V$ describes a group of $Y$-periodic test functions which satisfy homogenous Dirichlet boundary conditions, then the general elasticity problem for domain $\Omega$ can be stated as eq. (2.5).

$$
\begin{gathered}
\sigma_{i j}^{\epsilon}=E_{i j k l}^{\epsilon} \varepsilon_{k l}^{\epsilon} \\
\varepsilon_{k l}^{\epsilon}=\frac{1}{2}\left(\frac{\partial u_{k}^{\epsilon}}{\partial x_{l}}+\frac{\partial u_{l}^{\epsilon}}{\partial x_{k}}\right) \\
\int_{\Omega} E_{i j k l}^{\epsilon} \frac{\partial u_{k}^{\epsilon}}{\partial x_{l}} \frac{\partial v_{i}}{\partial x_{j}} d \Omega=\int_{\Omega} f_{i} v_{i} d \Omega+\int_{\Gamma_{t}} t_{i} v_{i} d \Gamma \quad \forall v_{i} \in V
\end{gathered}
$$


Where $\sigma_{i j}, \varepsilon_{i j}$ and $E_{i j k l}$ show the stress, strain and elasticity tensors. $u_{i}$ represents displacement field and same as eq. (2.2), $\epsilon$ superscript shows the periodicity of the given variable on global coordinate system $\boldsymbol{x}$.

Since solution for eq. (2.5) $\left(\boldsymbol{u}^{\epsilon}\right)$ depends on macro-scale and micro-scale domains (for different points in macroscopic domain microstructure varies, but if it is studied in microscopic level a periodic pattern could be detected [17]), an expansion in form of eq. (2.2) can be written for it:

$$
u_{i}^{\epsilon}(\boldsymbol{x})=u_{i}^{0}(\boldsymbol{x}, \boldsymbol{y})+\epsilon^{1} u_{i}^{1}(\boldsymbol{x}, \boldsymbol{y})+\epsilon^{2} u_{i}^{2}(\boldsymbol{x}, \boldsymbol{y})+\ldots
$$

Now by substituting eq. (2.6) back into eq. (2.5) and using chain rule of function differentiation which is:

$$
\frac{\partial(.)}{\partial x_{i}^{\epsilon}}=\frac{\partial(.)}{\partial x_{i}}+\frac{1}{\epsilon} \frac{\partial(.)}{\partial y_{i}}
$$

we will get:

$$
\begin{aligned}
\int_{\Omega} E_{i j k l}^{\epsilon}\left\{\frac{1}{\epsilon^{2}} \frac{\partial u_{k}^{0}}{\partial y_{l}} \frac{\partial v_{i}}{\partial y_{j}}+\frac{1}{\epsilon}\left[\left(\frac{\partial u_{k}^{0}}{\partial x_{l}}+\frac{\partial u_{k}^{1}}{\partial y_{l}}\right) \frac{\partial v_{i}}{\partial y_{j}}+\frac{\partial u_{k}^{0}}{\partial y_{l}} \frac{\partial v_{i}}{\partial x_{j}}\right]\right. \\
\left.+\left[\left(\frac{\partial u_{k}^{0}}{\partial x_{l}}+\frac{\partial u_{k}^{1}}{\partial y_{l}}\right) \frac{\partial v_{i}}{\partial x_{j}}+\left(\frac{\partial u_{k}^{1}}{\partial x_{l}}+\frac{\partial u_{k}^{2}}{\partial y_{l}}\right) \frac{\partial v_{i}}{\partial y_{j}}\right]+\epsilon(\ldots)\right\} d \Omega \\
=\int_{\Omega} f_{i} v_{i} d \Omega+\int_{\Gamma_{t}} t_{i} v_{i} d \Gamma
\end{aligned}
$$

Since eq. (2.8) has to be valid for any given $\epsilon \rightarrow 0^{+}$, it is necessary that coefficients of any power of $\epsilon$ be zero [50]. Therefore:

$$
\begin{gathered}
\frac{1}{\epsilon^{2}} \int_{\Omega} E_{i j k l}^{\epsilon} \frac{\partial u_{k}^{0}}{\partial y_{l}} \frac{\partial v_{i}}{\partial y_{j}} d \Omega=0 \\
\frac{1}{\epsilon} \int_{\Omega} E_{i j k l}^{\epsilon}\left[\left(\frac{\partial u_{k}^{0}}{\partial x_{l}}+\frac{\partial u_{k}^{1}}{\partial y_{l}}\right) \frac{\partial v_{i}}{\partial y_{j}}+\frac{\partial u_{k}^{0}}{\partial y_{l}} \frac{\partial v_{i}}{\partial x_{j}}\right] d \Omega=0 \\
\int_{\Omega} E_{i j k l}^{\epsilon}\left[\left(\frac{\partial u_{k}^{0}}{\partial x_{l}}+\frac{\partial u_{k}^{1}}{\partial y_{l}}\right) \frac{\partial v_{i}}{\partial x_{j}}+\left(\frac{\partial u_{k}^{1}}{\partial x_{l}}+\frac{\partial u_{k}^{2}}{\partial y_{l}}\right) \frac{\partial v_{i}}{\partial y_{j}}\right] d \Omega=\int_{\Omega} f_{i} v_{i} d \Omega+\int_{\Gamma_{t}} t_{i} v_{i} d \Gamma
\end{gathered}
$$


Multiplying eq. (2.9) by $\epsilon^{2}$, taking the limit $\epsilon \rightarrow 0^{+}$, integration by parts, applying divergence theorem and implementing periodic boundary conditions on opposing faces will lead to a boundary value problem of $\boldsymbol{u}^{0}$ in domain $Y[17]$ :

$$
-\frac{\partial}{\partial y_{j}}\left(E_{i j k l}^{\epsilon} \frac{\partial u_{k}^{0}}{\partial y_{l}}\right)=0 \quad y \in Y
$$

Which gives us:

$$
\boldsymbol{u}^{0}=\boldsymbol{u}^{0}(\boldsymbol{x})
$$

$\boldsymbol{u}^{0}$ is the first term in asymptotic expansion for displacement eq. (2.6) and it is independent of microscopic coordinates $\boldsymbol{y}$. In other words we can say asymptotic expansion divides behavior of the displacement field into two parts. $\boldsymbol{u}^{0}(\boldsymbol{x})$ which is only a function of global coordinates, represents the macroscopic or homogenized part of the displacement and rest of the terms in eq. (2.6) carry the microscopic effects. Now by using information in eq. (2.13), multiplying eq. (2.10) by $\epsilon$ and taking the limit $\epsilon \rightarrow 0^{+}$we will have:

$$
\int_{Y} E_{i j k l}^{\epsilon}\left(\frac{\partial u_{k}^{0}}{\partial x_{l}}+\frac{\partial u_{k}^{1}}{\partial y_{l}}\right) \frac{\partial v_{i}}{\partial y_{l}} d Y=0
$$

Following the steps performed on eq. (2.9), which were integration by part, using divergence theorem and applying periodic boundary conditions, eq. (2.14) could be represented in the form of a boundary value problem which should be solved for $\boldsymbol{u}^{1}$ [50]:

$$
\frac{\partial}{\partial y_{j}}\left[E_{i j k l}^{\epsilon}\left(\frac{\partial u_{k}^{0}}{\partial x_{l}}+\frac{\partial u_{k}^{1}}{\partial y_{l}}\right)\right]=0
$$

It has been proposed that the solution for eq. (2.15) takes the form of $[8,17]$ :

$$
u_{i}^{1}=-\chi_{i}^{k l}(\boldsymbol{x}, \boldsymbol{y}) \frac{\partial u_{k}^{0}(\boldsymbol{x})}{\partial x_{l}}+\tilde{u}_{i}^{1}(\boldsymbol{x})
$$

Where $\tilde{u}_{i}^{1}(\boldsymbol{x})$ is an arbitrary integration constant in $\boldsymbol{y}, \chi^{k l} \in V$ are test functions satisfying periodicity conditions and since eq. (2.15) is linear with respect to $\boldsymbol{u}^{0}$ 
they can be the solution for [17]:

$$
\int_{Y} E_{i j p m} \frac{\partial \chi_{p}^{k l}}{\partial y_{m}} \frac{\partial v_{i}}{\partial y_{j}} d Y=\int_{Y} E_{i j k l} \frac{\partial v_{i}}{\partial y_{j}} d Y \quad \forall v_{i} \in V
$$

In the next step if we take the limit $\epsilon \rightarrow 0^{+}$of eq. (2.11) and use eq. (2.13) in conjunction with following lemma which is valid for a $Y$-periodic function $f(\boldsymbol{y})$ :

$$
\lim _{\epsilon \rightarrow 0^{+}} \int_{\Omega} f\left(\frac{\boldsymbol{x}}{\epsilon}\right) d \Omega \rightarrow \frac{1}{|Y|} \int_{\Omega} \int_{Y} f(\boldsymbol{y}) d Y d \Omega
$$

We will get the macro-scale balance equation:

$$
\begin{aligned}
& \int_{\Omega}\left[\frac{1}{|Y|} \int_{Y} E_{i j k l}\left(\frac{\partial u_{k}^{0}}{\partial x_{l}}+\frac{\partial u_{k}^{1}}{\partial y_{l}}\right) d Y\right] \frac{\partial v_{i}}{\partial x_{j}} d \Omega \\
= & \int_{\Omega}\left(\frac{1}{|Y|} \int_{Y} f_{i} d Y\right) v_{i} d \Omega+\int_{\Gamma_{t}} t_{i} v_{i} d \Gamma \quad \forall v_{i} \in V
\end{aligned}
$$

Here $|Y|$ means volume or area for three or two dimensional domains. Now substituting eq. (2.16) into eq. (2.19) yields to:

$$
\int_{\Omega} E_{i j k l}^{H} \frac{\partial u_{0}}{\partial x_{l}} \frac{\partial v_{i}}{\partial x_{j}} d \Omega=\int_{\Omega} \bar{f}_{i} v_{i} d \Omega+\int_{\Gamma_{t}} t_{i} v_{i} d \Gamma \quad \forall v_{i} \in V
$$

Where:

$$
\begin{gathered}
E_{i j k l}^{H}=\frac{1}{|Y|} \int_{Y}\left(E_{i j k l}-E_{i j p m} \frac{\partial \chi_{p}^{k l}}{\partial y_{m}}\right) d Y \\
\bar{f}_{i}=\frac{1}{|Y|} \int_{Y} f_{i} d Y
\end{gathered}
$$

Comparing eq. (2.20) to eq. (2.5) denotes the fact that eq. (2.20) is macroscopic balance equation and $E_{i j k l}^{H}$ defined by eq. (2.21) represents the homogenized elasticity tensor and $\bar{f}_{i}$ is the average body force.

Going back to eq. (2.17) and putting it side by side eq. (2.20) shows that microscale and macro-scale equations are not coupled and homogenized elasticity tensor can be computed by solving eq. (2.17) and the solution is completely independent of macro-scale displacement $\boldsymbol{u}^{0}$. So for a given inhomogeneous material as long as 
the geometry of RVE does not change, homogenized elasticity tensor calculated for it is going to be valid for macroscopic problems with different external loads and boundary conditions.

It also should be noted that in eq. (2.17), $\boldsymbol{\chi}$ functions are symmetric with respect to $k$ and $l$ indices which means that for a three dimensional problem six equations in the form of eq. (2.17) should be solved and a two dimensional problem will only require three equations with different $k l$ indices [17].For a 3D problem $k l=11,22,33,12 / 21,13 / 31,23 / 32$ and in a $2 \mathrm{D}$ domain $k l=11,22,12 / 21$.

Here in this work a first order asymptotic expansion will be used for displacement and therefore the final form of eq. (2.6) will be:

$$
\boldsymbol{u}^{\epsilon}(\boldsymbol{x})=\boldsymbol{u}^{0}(\boldsymbol{x})-\epsilon\left(\chi_{i}^{k l}(\boldsymbol{x}, \boldsymbol{y}) \frac{\partial u_{k}^{0}(\boldsymbol{x})}{\partial x_{l}}-\tilde{u}_{i}^{1}(\boldsymbol{x})\right)+\epsilon^{2}(\ldots)
$$

Higher order displacement correctors can be calculated by following similar steps, interested reader can refer to [50] and [20] for more information.

\subsubsection{Stress distribution}

After calculation of homogenized elasticity tensor and finding the formulation for the terms in displacement asymptotic expansion shown in eq. (2.6), we can use those information to investigate stress and strain distribution in micro level. As mentioned in Section 2.1.1 one of the main advantages of asymptotic method is that it can provide the solution for full structural problem at micro level and unlike other homogenization techniques, this method provides a localization algorithm to explicitly define the equation for stress and strain at micro-scale [28].

If we go back to eq. (2.6) and write a first order expansion for displacement, substitute it in eq. (2.4) and use the chain rule represented in eq. (2.7) we will get:

$$
\varepsilon_{i j}^{\epsilon}(\boldsymbol{x})=\epsilon^{-1} \varepsilon_{i j}^{-1}(\boldsymbol{x}, \boldsymbol{y})+\epsilon^{0} \varepsilon_{i j}^{0}(\boldsymbol{x}, \boldsymbol{y})+\epsilon^{1} \varepsilon_{i j}^{1}(\boldsymbol{x}, \boldsymbol{y})
$$


Where:

$$
\begin{gathered}
\varepsilon_{i j}^{-1}=\frac{1}{2}\left(\frac{\partial u_{i}^{0}}{\partial y_{j}}+\frac{\partial u_{j}^{0}}{\partial y_{i}}\right) \\
\varepsilon_{i j}^{0}=\frac{1}{2}\left(\frac{\partial u_{i}^{0}}{\partial x_{j}}+\frac{\partial u_{j}^{0}}{\partial x_{i}}\right)+\frac{1}{2}\left(\frac{\partial u_{i}^{1}}{\partial y_{j}}+\frac{\partial u_{j}^{1}}{\partial y_{i}}\right) \\
\varepsilon_{i j}^{1}=\frac{1}{2}\left(\frac{\partial u_{i}^{1}}{\partial x_{j}}+\frac{\partial u_{j}^{1}}{\partial x_{i}}\right)+\frac{1}{2}\left(\frac{\partial u_{i}^{2}}{\partial y_{j}}+\frac{\partial u_{j}^{2}}{\partial y_{i}}\right)
\end{gathered}
$$

Knowing that $\boldsymbol{u}^{0}$ is only a function of global coordinate $\boldsymbol{x}$ (2.13) and the fact that we are only using first order expansion, eq. (2.24) can be rewritten in the following form which describes the localized strains:

$$
\varepsilon_{i j}^{\epsilon}=\varepsilon_{i j}^{0}+\epsilon^{1}(\ldots) \approx \frac{1}{2}\left[\left(\frac{\partial u_{i}^{0}}{\partial x_{j}}+\frac{\partial u_{j}^{0}}{\partial x_{i}}\right)+\left(\frac{\partial u_{i}^{1}}{\partial y_{j}}+\frac{\partial u_{j}^{1}}{\partial y_{i}}\right)\right]
$$

Now by substituting eq. (2.28) back into eq. (2.3) the equation for localized stresses can also be derived:

$$
\sigma_{i j}^{\epsilon}=\sigma_{i j}^{0}+\epsilon^{1}(\ldots) \approx \frac{1}{2} E_{i j k l}^{\epsilon}\left[\left(\frac{\partial u_{k}^{0}}{\partial x_{l}}+\frac{\partial u_{l}^{0}}{\partial x_{k}}\right)+\left(\frac{\partial u_{k}^{1}}{\partial y_{l}}+\frac{\partial u_{l}^{1}}{\partial y_{k}}\right)\right]
$$

\subsubsection{Validation of homogenized properties}

In Section 2.1.1 detailed formulation for calculation of homogenized mechanical properties with asymptotic method was presented, here we are going to introduce two alternate methods for computation of such properties. Later depending on the type of the numerical experiments which we are conducting, one or both of these methods are going to be used to evaluate the accuracy of the results of our computational platform.

\section{Hashin-Shtrikman bounds}

As mentioned in Chapter 1, over the last decades different methods have been developed to calculate the effective properties of inhomogeneous materials. Among 
them there is a group of methods which can provide an upper and lower bound to predict the homogenized properties of a composite structure. Hashin and Shtrikman bounds are one of the very common approaches for dealing with effective properties. This method was developed based on a variational approach and it is valid for materials with linear constitutive behavior and an isotropic microstructure regular enough which could be considered as a periodic structure [18, 19].

Based on Hashin and Shtrikman's formulation, the upper and lower bounds for bulk and shear modulus of a two phase microstructure is defined as:

$$
\begin{array}{r}
\kappa_{1}+\frac{f_{2}}{\frac{1}{\kappa_{2}-\kappa_{1}}+\frac{3\left(1-f_{2}\right)}{3 \kappa_{1}+4 \mu_{1}}} \leq \kappa^{*} \leq \kappa_{2}+\frac{1-f_{2}}{\frac{1}{\kappa_{1}-\kappa_{2}}+\frac{3 f_{2}}{3 \kappa_{2}+4 \mu_{2}}} \\
G_{1}+\frac{f_{2}}{\frac{1}{G_{2}-G_{1}}+\frac{6\left(1-f_{2}\right)\left(\kappa_{1}+2 G_{1}\right)}{5 G_{1}\left(3 \kappa_{1}+4 G_{1}\right)}} \leq G^{*} \leq G_{2}+\frac{\left(1-f_{2}\right)}{\frac{1}{G_{1}-G_{2}}+\frac{6 f_{2}\left(\kappa_{2}+2 G_{2}\right)}{5 G_{2}\left(3 \kappa_{2}+4 G_{2}\right)}}
\end{array}
$$

Where $\kappa^{*}$ and $G^{*}$ are the homogenized bulk and shear modulus, $\kappa_{1}, G_{1}$ and $\kappa_{2}, G_{2}$ are the modulus for two phases of material, $f_{2}$ is the volume fraction of phase two and it is also assumed that $\kappa_{2} \geq \kappa_{1}$ and $G_{2} \geq G 1$ [35]. Bulk and shear modulus can be defined in terms of elasticity tensor elements:

$$
\kappa=\frac{1}{2}\left(C_{1111}+C_{1122}\right) \quad G=\frac{1}{2}\left(C_{1111}-C_{1122}\right)
$$

And in two dimensional problems for plane stress and plane strain special cases eq. (2.32) can be simplified to:

$$
\begin{array}{cl}
\kappa=\frac{E}{2(1-\nu)} & \text { For plane stress } \\
\kappa=\frac{E}{2(1+\nu)(1-2 \nu)} & \text { For plane strain } \\
G=\frac{E}{2(1+\nu)} & \text { For plane stress and plane strain }
\end{array}
$$

Although Hashin-Shtrikman bounds do not provide the exact values of the homogenized properties, but they can offer an acceptable region of results for overall properties of material structures which are compatible with their assumptions. 


\section{Strain energy method}

Strain energy method is based on Hill's theory which provides all the necessary and sufficient conditions for energetically and mechanically defined effective properties to be equal to each other. For a linear elastic material, this principle can be outlined as $[26]$ :

$$
\langle\boldsymbol{\sigma}: \varepsilon\rangle=\langle\boldsymbol{\sigma}\rangle:\langle\varepsilon\rangle
$$

Where $\boldsymbol{\sigma}$ and $\boldsymbol{\varepsilon}$ are stress and strain tensors, $\langle$.$\rangle means spatial average in the domain$ and ":" shows the twice contracted tensor product. Thus, according to eq. (2.34) the average of the products equals the product of averages.

For a better understanding of Hill's principle let us consider a body $B$ with a known microstructure. Due to some boundary conditions on $\partial B$ and in absence of any body forces, there are stress and strain fields $\boldsymbol{\sigma}$ and $\varepsilon$ induced in $B$. If we show these fields as a superposition of the mean values and zero-mean fluctuations [48]:

$$
\boldsymbol{\sigma}=\overline{\boldsymbol{\sigma}}+\boldsymbol{\sigma}^{\prime} \quad \varepsilon=\bar{\varepsilon}+\varepsilon^{\prime}
$$

we can define the volume average of energy density in $B$ as:

$$
\langle U\rangle=\frac{1}{2 V} \int_{B} \boldsymbol{\sigma}: \varepsilon d V=\frac{1}{2}\langle\boldsymbol{\sigma}: \boldsymbol{\varepsilon}\rangle=\frac{1}{2}\langle\boldsymbol{\sigma}\rangle:\langle\boldsymbol{\varepsilon}\rangle+\frac{1}{2}\left\langle\boldsymbol{\sigma}^{\prime}: \boldsymbol{\varepsilon}^{\prime}\right\rangle
$$

Therefore, for the average of products to be equal to the product of averages we should have:

$$
\left\langle\boldsymbol{\sigma}^{\prime}: \varepsilon^{\prime}\right\rangle=0
$$

For an infinite domain this condition is trivially satisfied, but in a finite body, for eq. (2.37) to be true the body has to be loaded in a specific way on its boundaries. By using Gauss-Green theorem, the necessary and sufficient conditions for eq. (2.37) to hold could be found [25, 24]:

$$
\langle\boldsymbol{\sigma}: \boldsymbol{\varepsilon}\rangle=\langle\boldsymbol{\sigma}\rangle:\langle\boldsymbol{\varepsilon}\rangle \Leftrightarrow \int_{\partial B}(\boldsymbol{t}-\langle\boldsymbol{\sigma}\rangle \cdot \boldsymbol{n}) \cdot(\boldsymbol{u}-\langle\boldsymbol{\varepsilon}\rangle \cdot \boldsymbol{x}) d S=0
$$


Where $\boldsymbol{t}$ is traction, $\boldsymbol{u}$ is displacement, $\boldsymbol{n}$ and $\boldsymbol{x}$ respectively show the normal vector to the boundary and the coordinates of boundary points. Looking back to eq. (2.38) it could be determined that there are three different types of boundary conditions which satisfy this equation.

First group of these boundary conditions is Dirichlet type which is usually called Kinematic Uniform Boundary Conditions or KUBC and it can be represented as:

$$
\boldsymbol{u}(\boldsymbol{x})=\varepsilon^{0} \cdot \boldsymbol{x} \quad \forall \boldsymbol{x} \in \partial B
$$

Next class of boundary conditions which satisfy eq. (2.38) is a group of Neumann conditions named Static Uniform Boundary Conditions or SUBC:

$$
\boldsymbol{t}(\boldsymbol{x})=\sigma^{0} \cdot \boldsymbol{n} \quad \forall \boldsymbol{x} \in \partial B
$$

There is also another set of boundary conditions which can make the integral in eq. (2.38) to be equal to zero called Mixed Uniform Boundary Conditions or MUBC:

$$
\left(\boldsymbol{t}(\boldsymbol{x})-\sigma^{0} \cdot \boldsymbol{n}\right) \cdot\left(\boldsymbol{u}(\boldsymbol{x})-\varepsilon^{0} \cdot \boldsymbol{x}\right)=0 \quad \forall \boldsymbol{x} \in \partial B
$$

Different combination of traction and displacement boundary conditions can be combined together to create a mixed boundary condition of form eq. (2.41). Considering the fact that here we are dealing with homogenization problems, two sets of these combinations are of great importance, displacement periodic boundary condition:

$$
\begin{aligned}
& \boldsymbol{u}(\boldsymbol{x})=\varepsilon^{0} . \boldsymbol{x} \quad \forall \boldsymbol{x} \in \partial B_{\text {displacement }} \\
& \boldsymbol{u}(\boldsymbol{x}+\boldsymbol{L})=\boldsymbol{u}(\boldsymbol{x})+\varepsilon^{0} . \boldsymbol{L}, \quad \boldsymbol{t}(\boldsymbol{x}+\boldsymbol{L})=-\boldsymbol{t}(\boldsymbol{x}) \quad \forall \boldsymbol{x} \in \partial B_{\text {periodic }}
\end{aligned}
$$

And traction periodic boundary condition:

$$
\begin{aligned}
& \boldsymbol{t}(\boldsymbol{x})=\sigma^{0} \cdot \boldsymbol{x} \quad \forall \boldsymbol{x} \in \partial B_{\text {traction }} \\
& \boldsymbol{u}(\boldsymbol{x}+\boldsymbol{L})=\boldsymbol{u}(\boldsymbol{x})+\varepsilon^{0} . \boldsymbol{L}, \quad \boldsymbol{t}(\boldsymbol{x}+\boldsymbol{L})=-\boldsymbol{t}(\boldsymbol{x}) \quad \forall \boldsymbol{x} \in \partial B_{\text {periodic }}
\end{aligned}
$$


In eqs. (2.39) to (2.43), $\boldsymbol{L}$ is the periodic length. $\sigma^{0}$ and $\varepsilon^{0}$ show the prescribed uniform tensors on the boundary and it should be noted that according to average stress and strain theory $\varepsilon^{0}=\langle\boldsymbol{\varepsilon}\rangle$ and $\sigma^{0}=\langle\boldsymbol{\sigma}\rangle$ [48]. By applying different sets of any of the introduced boundary conditions along different coordinate axis, strain energy formula can be used to determine different elements of the elasticity tensors. More detailed explanation on methods of applying the boundary conditions could be found in [48], [25], [24], [30] and [23].

\section{$2.2 \quad$ MeshFree}

As mentioned in Chapter 1, over the last decade meshfree or generally mesh reduction methods have introduced themselves as powerful numerical tools for solving a vast range of science and engineering problems with the purpose of eliminating meshing difficulties which exist in current finite element methods. Meshfree techniques have been applied to different categories of problems like crack propagation [2], moving material interface [59], incompressible fluid flow [66], vibration [37] and also problems with highly oscillatory solutions such as multiscale problems [29].

In this section we will go over the basic concept of a meshfree solution and will explain the necessary tools and techniques for development of a meshfree computational platform.

\subsubsection{Solution Structure Method}

In many engineering problems, the governing equations are presented in the form of a partial differential equation and such equation over a domain $\Omega$ can be generally 
formulated as [59]:

$$
\begin{aligned}
& A u=f \\
& \left.L_{i} u\right|_{\partial \Omega_{i}}=\varphi_{i} \\
& \left.u\right|_{t=0}=u_{0} \quad \frac{\partial u}{\partial t}=\dot{u}_{0}
\end{aligned}
$$

Where operator $A$ describes the general physical law, $u(\boldsymbol{x}, t)$ is a unknown function representing the distribution of a physical field in space and time and $f$ is some known function. Values of function $u$ or its derivatives or both are defined by operator $L_{i}$ and given function $\varphi_{i}$ on the $i$ th portion of the boundary $\partial \Omega$ of a given domain $\Omega$. And if the domain, its boundaries or the boundary conditions can vary with time, the initial conditions for the transient problem can include the initial values of the function or its derivatives.

Exact solution for boundary value problem (BVP) presented in eq. (2.44) exist only for very few and very simple equations. Real world problems with complex geometrical domain representations are mostly solved approximately by one of the many known numerical methods.

The numerical method utilized in this dissertation for solving boundary value problems is called Solution Structure Method (SSM) which is in fact a generalized form of $R$-function method (RFM) developed by Rvachev and his students [53, 54]. RFM itself is an extension of the approach introduced by Kantorovich for solving two dimensional homogenous boundary value problems with Dirichelt boundary conditions and the idea behind it is based on the observation that the solution of such problem can be presented in form of [36]:

$$
u=\omega \Phi
$$

Where $\Phi$ is some unknown function and $\omega: R^{n} \rightarrow R$ is a known function which has zero values on the boundary of the domain $\partial \Omega$ and it is positive inside $\Omega$. Also $\omega$ 
functions are designed in such way that they are normalized to $m$-th order:

$$
\left.\omega\right|_{\partial \Omega}=0 \quad,\left.\quad \frac{\partial \omega}{\partial n}\right|_{\partial \Omega}=1 \quad,\left.\quad \frac{\partial^{k} \omega}{\partial n^{k}}\right|_{\partial \Omega}=0 \quad k=2,3, \ldots, m
$$

Expression eq. (2.45) contains two independent type of information: the function $\omega$ describing all the geometric information of this specific boundary value problem and function $\Phi$ which is responsible for satisfying the differential equation of the boundary value problem. Since $\omega$ is exactly zero on the boundary $\partial \Omega$, any solution in the form of eq. (2.45) will exactly satisfy the homogenous boundary conditions and this is completely independent of the unknown function $\Phi$ or the type of differential equation $[59,56]$.

Since determination of function $\Phi$ satisfying the differential equation is not analytically always possible, we can approximate it by a finite linearly independent series [59]:

$$
\Phi=\sum_{i=1}^{n} C_{i} \chi_{i}
$$

Where $C_{i}$ 's are scalar coefficients and $\chi_{i}$ 's are a group of basis functions. Solution structure presented in eq. (2.45) does not place any constraints on $\chi$ functions and the choice of these functions are completely independent of any spatial discretization of the domain [56].

Substituting eq. (2.47) back into eq. (2.45) will give us the structure of approximate numerical solution to a boundary value problem with homogeneous Dirichlet boundary conditions in the from of:

$$
u=\omega \sum_{i=1}^{n} C_{i} \chi_{i}
$$

and any type of numerical method such as variational, projection, energy function minimization, etc., can be used to solve for the numerical values of $C_{i}$.

After the initial introduction of solution structure eq. (2.45), the SSM concept was developed by Rvachev and his team for boundary value problems with different 
Table 2.1: Solution structures corresponding to different types of boundary conditions for second order partial differential equations [64].

\begin{tabular}{lll}
\hline Type of BC & BC Formulation & Solution Structure \\
\hline Dirichlet & $\left.u\right|_{\partial \Omega}=\varphi$ & $u=\omega \Phi+\varphi$ \\
Neumann & $\left.\frac{\partial u}{\partial n}\right|_{\partial \Omega}=\varphi$ & $u=\Phi-\omega D_{1}^{\omega}(\Phi)-\omega \Phi+\omega \varphi+\omega^{2} \Phi$ \\
3rd kind & $\left.\left(\frac{\partial u}{\partial n}+h u\right)\right|_{\partial \Omega}=\varphi$ & $u=\Phi-\omega D_{1}^{\omega}(\Phi)-h \omega \Phi+\omega \varphi+\omega^{2} \Phi$ \\
Mixed & $\left.u\right|_{\partial \Omega_{1}}=\varphi$ & \\
& $\left.\left(\frac{\partial u}{\partial n}+h u\right)\right|_{\partial \Omega_{2}}=\psi$ & $\frac{\omega_{1} \omega_{2}}{\omega_{1}+\omega_{2}}\left(\psi+\omega_{2} \Phi-D_{1}^{\omega_{2}}\left(\omega_{1} \Phi+\varphi\right)-h \omega_{1} \Phi-h \varphi\right)$ \\
& &
\end{tabular}

types of boundary conditions. A summary of proposed solution structures are presented in Table 2.1. Mathematical formulation for derivation of such structures are explained in details in [56] and [64].

In Table 2.1 each solution structure is in fact a function made from the unknown $\Phi$ function, normalized $\omega_{i}$ functions describing the geometry of domain boundaries, prescribed functions $\psi, h, \varphi$ and the operator $D_{i}$ which is defined as:

$$
D_{i}^{\omega}(.)=(\nabla(.) \cdot \nabla \omega)^{i}
$$

As mentioned earlier $\Phi$ functions are finite linear combination of basis functions which can be selected from B-splines, polynomials, trigonometric polynomials or any other common choices and regardless of the chosen system of basis functions all these solution structures will satisfy boundary conditions exactly. In this method, not like traditional PDE solvers which require discretization of the geometry and applying boundary conditions at the nodes, satisfaction of boundary conditions is done by innovative design of $\omega$ functions. 


\subsubsection{Distance field functions}

The constructional details of solution structure for a boundary value problem were discussed in Section 2.2.1 and based on what was delivered it can easily be observed that creation of a solution structure strongly depends on the ability to construct a function $\omega$ that vanishes on all or a portion of the boundary of the geometric domain. In other words it could be said that equation $\omega=0$ should define the domain boundaries.

One group of functions which can provide such properties are exact distance field functions, which measure the normal distance of any given point in the domain to the closest boundary. By definition, these functions are differentiable and have unit gradients everywhere except the points that are equidistance from several boundaries (for example points on medial axis or corner points). Also the zero set of exact distance field functions corresponds to and implicitly defines the boundaries of a geometric domain [58].

Since differential properties of $\omega$ functions are critical in many applications including construction of a solution structure for a boundary value problem, it was proposed to replace exact distance field functions with their $m$-th order approximation to overcome their differentiability limitations $[59,6]$.

Different methods have been used for generation of approximate distance field functions such as fitting or interpolation of splines and radial basis functions [67], applying level set methods [71] or taking advantage of constructive methods like theory of $R$-functions $[53,54]$.

Theory of $R$-functions can provide the necessary tools for construction of approximate distance field functions which can implicitly represent a domain [59]. An $R$-function is a real-valued function whose sign is completely determined by the signs

of its arguments, for example $f\left(x_{1}, x_{2}, x_{3}\right)=x_{1} x_{2} x_{3}$ can be negative only when the 
number of its negative arguments are odd. In other words a $R$-function works as a boolean switching function changing its sign only when their arguments change their signs [53]. The main advantage of the theory relies on the fact that every formal logical sentence has an equivalent class of $R$-functions which their signs are determined by the truth table of the logical sentence. Two of the most popular $R$-functions are:

$$
\begin{aligned}
& x_{1} \wedge_{\alpha} x_{2}=\frac{1}{1+\alpha}\left(x_{1}+x_{2}-\sqrt{x_{1}^{2}+x_{2}^{2}-2 \alpha x_{1} x_{2}}\right) \\
& x_{1} \vee_{\alpha} x_{2}=\frac{1}{1+\alpha}\left(x_{1}+x_{2}+\sqrt{x_{1}^{2}+x_{2}^{2}-2 \alpha x_{1} x_{2}}\right)
\end{aligned}
$$

Which corresponds to the logical conjunction $\wedge$ and disjunction $\vee$ respectively and $\alpha\left(x_{1}, x_{2}\right)$ is an arbitrary function with $-1<\alpha\left(x_{1}, x_{2}\right)<1$. Even the value of $\alpha$ can be set to a constant, for example $\alpha=1$ yields to min and max functions or setting $\alpha=0$ results in a function which is analytical everywhere except at $x_{1}=x_{2}=0$. As boolean functions, $R$-functions could be used to describe any region represented by a system of inequalities as a single function $\omega$ which can be normalized up to $m$-th order. Interested reader can refer to [54], [59], [58] and [6] for extensive studies on approximate distance fields created with $R$-functions and their differential properties.

For a better understanding of the concept, here as an example we will explain different steps of constructing approximate distance field functions for a simple geometry. Let us assume that the geometry of a sample domain is a right angled triangle shown in Fig. 2.3(a). To represent this domain in terms of approximate distance fields first it is broken down to intersection of three primitive half-spaces which can easily be described by simple inequalities:

$$
\omega_{1}(x, y)=x-y \geq 0 \quad \omega_{2}(x, y)=a-x \geq 0 \quad \omega_{3}(x, y)=y \geq 0
$$


(a)

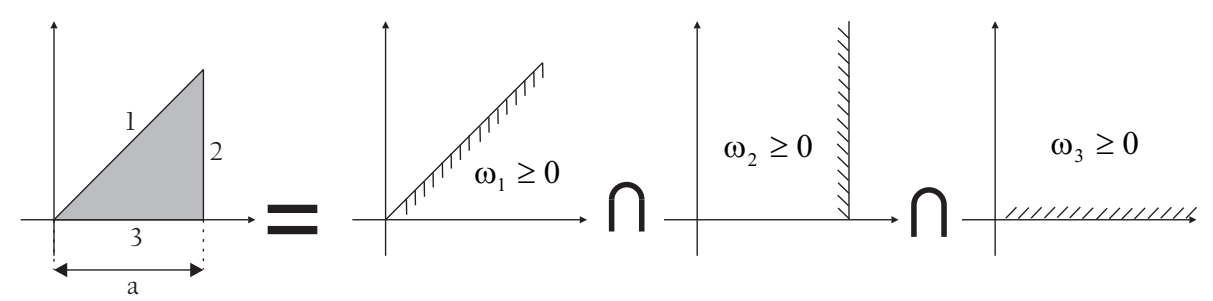

(b)

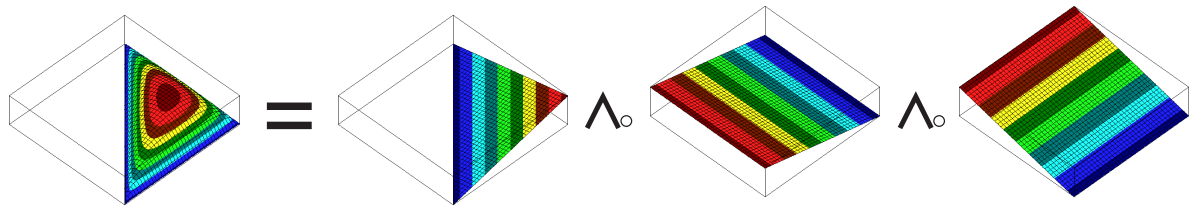

Figure 2.3: (a) The native geometry, a right angled triangle, broken down into intersection of three primitive half spaces; (1) under line 1, (2) left side of line 2 and (3) above line 3. (b) Inequalities for $\omega_{1}, \omega_{2}$ and $\omega_{3}$ plotted as functions of $x$ and $y$ and the resulting $R_{0}$-conjunction $\omega=\omega_{1} \wedge_{0} \omega_{2} \wedge_{0} \omega_{3}$. The $\omega$ function has zero values and non-zero gradients on the boundaries and it is differentiable in the whole domain.

and $\omega$ will be:

$$
\omega=\left(\omega_{1} \geq 0\right) \cap\left(\omega_{2} \geq 0\right) \cap\left(\omega_{3} \geq 0\right)
$$

Now by using theory of $R$-functions and replacing boolean operators with their corresponding $R_{0}$-functions, approximate distance fields for the native geometry can be written as:

$$
\omega=\omega_{1} \wedge_{0} \omega_{2} \wedge_{0} \omega_{3}
$$

Fig. 2.3(b) shows the corresponding three dimensional plot for each of half-spaces and also the resulting conjunction of all of them which gives us the approximate distance field representation for the domain in question.

\subsubsection{Automatic differentiation and Integration}

After presenting the theoretical concepts for construction of solution structures and approximate distance field functions, we can actually implement the SSM for solving a boundary value problem and study the solution steps in a more practical manner. 
To do so, let us consider a simple boundary value problem involving Poisson equation and homogeneous Dirichlet boundary conditions which is defined over domain $\Omega$ :

$$
-\nabla^{2} f=K \quad, \quad K=\text { cte } \quad,\left.\quad f\right|_{\partial \Omega}=0
$$

We also assume that the approximate distance field functions $\omega$ describing domain $\Omega$ is already constructed by one of the methods explained in Section 2.2.2. Going back to Table 2.1, shows that based on presented boundary conditions the proper solution structure for this problem would be $f=\omega \Phi, \Phi=\sum_{i=1}^{n} C_{i} \chi_{i}$. Now substituting the solution structure back into Poisson equation, using Galerkin method with same test functions as basis functions in our solution structure and applying divergence theorem will give us:

$$
\int_{\Omega} \nabla\left[\sum_{j=1}^{n} C_{j}\left(\omega \chi_{j}\right)\right] \cdot \nabla\left(\omega \chi_{i}\right) d \Omega=\int_{\Omega} K \cdot \nabla\left(\omega \chi_{i}\right) d \Omega
$$

Differentiating eq. (2.55) with respect to the unknown coefficients $C_{j}$ yields to a system of algebraic equation $[A][C]=[B]$ where elements of matrix $A$ and vector $B$ are defined as:

$$
a_{i j}=\int_{\Omega} \nabla\left(\omega \chi_{i}\right) \cdot \nabla\left(\omega \chi_{j}\right) d \Omega \quad, \quad b_{j}=K \int_{\Omega} \nabla\left(\omega \chi_{j}\right) d \Omega
$$

Solving a boundary value problem with SSM will require differentiating the solution structure, integrating over the geometric domain and solving a system of linear

algebraic equations. Except for the solution of algebraic equation, rest of the computational techniques are rather different than the ones implemented in conventional mesh-based methods [64].

\section{Automatic differentiation}

The most important issue in calculating the partial derivatives of a solution structure in a system such as eq. (2.56) is that the domain dependent distance field 
functions are defined at run time, therefore a differentiation algorithm is required which can handle run time calculations with an acceptable accuracy. Many algorithmic differentiation methods have been formulated, but generally they can be divided into three main categories: Symbolic, Numerical, Automatic.

Symbolic differentiation can be used when the closed form of function is available and by date it has been implemented in many commercial softwares such as Maple and MATLAB, but unfortunately when the function is not a relatively simple one this method can become very intensive and unmanageable. Numerical methods are mainly based on finite difference method or approximating a function by polynomials or splines and since they only require evaluation of the function itself they have a clear advantage compared to the other methods, but sometimes they can become unstable and reaching to the desired accuracy might not be possible [60, 59].

Automatic differentiation is in fact a collection of techniques to evaluate the numerical value of a function's derivative at a certain point with a computer program. It uses same differentiation rules as symbolic method, but instead of propagating the symbolic expression of the derivatives it deals with numerical values of them and it can be implemented in forward or reverse modes. Detailed mathematical formulation of both forward and reverse automatic differentiation algorithms can be found in $[60,52]$.

In this dissertation, a forward automatic differentiation technique is utilized which uses Leibnitz rules to compute numeric values of partial derivatives and stores them in a certain type of data structure called tuple. The proposed data structure can provide a real time access to the values of derivatives and accelerate the whole computation process [63].

A tuple structure is a storage method which arranges the partial derivatives of 
a function (up to a certain order) in an array by increasing order of derivatives:

$$
\left[f(\boldsymbol{x}), \frac{\partial f}{\partial x_{1}}(\boldsymbol{x}), \ldots, \frac{\partial f}{\partial x_{n}}(\boldsymbol{x}), \frac{\partial^{2} f}{\partial^{2} x_{1}}(\boldsymbol{x}), \frac{\partial^{2} f}{\partial x_{1} \partial x_{2}}(\boldsymbol{x}), \ldots, \frac{\partial^{2} f}{\partial^{2} x_{n}}(\boldsymbol{x}), \ldots, \frac{\partial^{m} f}{\partial^{m} x_{n}}(\boldsymbol{x})\right]
$$

A powerful $\mathrm{C}++$ class for tuples have been developed which has elementary and arithmetic operations as friend functions and given a point in space, it is capable of calculating partial derivatives of a function up to a certain order and storing them in a structure such as eq. (2.57). By using such allocation scheme partial derivatives of same order will occupy the same spot in the storage array and they can be easily accessed just by pointing to the corresponding array index [62, 63, 64].

\section{Geometrically adaptive numerical integration}

In the process of solving a boundary value problem with solution structure method and after finding the numerical values of derivatives in a system similar to eq. (2.56), the next logical step would be evaluating the integrals over the geometric domain. Conventional numerical integration over solid domains usually requires generation of geometry conforming grid and adapting the solid boundaries to grid lines or faces. In this approach integration cells intersecting domain boundaries are either included or ignored. This can lead to considerable integration errors near the boundaries which is completely undesirable when it comes to solving boundary value problems.

A novel technique for calculation of integrals in boundary cells were proposed in [39] where the authors used a classification system for boundary cells in conjunction with parametrization of their geometry and they were able to allocate integration points in boundary cells using the exact geometry instead of statistical methods.

The idea of domain geometry parametrization is based on the fact that using a suitable coordinate system can eliminate singularities in integrands and reduce the integration error. In this method, geometrically adaptive integration, for each 

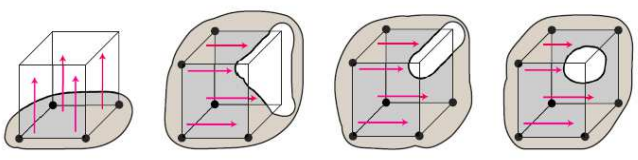

(a)

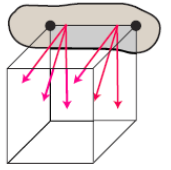

(b)

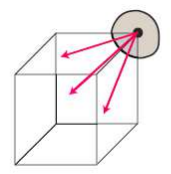

(c)

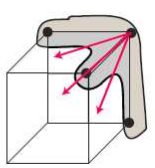

Figure 2.4: (a) Cartesian coordinates used for boundary cells with at least one face fully contained in the domain (b) Cylindrical coordinates for boundary cells with at least on edge in the domain (c) Spherical coordinates for boundary cells with at least one vertex in the domain [39].

boundary cell depending on the number of its faces, edges or vertexes being in the domain one of the Cartesian, cylindrical or spherical coordinate systems is assigned to parameterize the cell geometry. Fig. 2.4 shows some of the different possible arrangements of boundary and boundary cells and suitable coordinate system for each combination.

By choosing a proper coordinate system and having a characteristic function representing the domain, geometrically adaptive integration can provide a more efficient distribution of integration points in geometric cells and as a result lead to a better numerical accuracy for the same computational cost compared to other integration methods.

\subsubsection{Image Acquisition}

The importance of constructing approximate distance field functions as one of the essential ingredients of solution structure method was discussed in Section 2.2.2 and it was explained how theory of $R$-functions can be used to generate such functions. Here we will discuss the fact that constructing distance field functions are not restricted to simple boundary representation of geometric domains and it can be applied for modeling of much more complex geometries. 
In real life engineering problems which often involve complex geometries, different methods can be used to describe the domain such as CAD models, point clouds, polygonal meshes, B-splines, etc. It was shown that not only approximate distance fields can be generated from any of the mentioned domain representations, but also using this method will lead to a more automated field modeling process compared to conventional mesh based methods [11].

This method proposes to represent approximate distance field function $\omega$ in the form of a finite linear combination of basis functions:

$$
\omega(x, y, z)=\sum_{i=1}^{m} C_{i}^{\omega} \chi_{i}^{\omega}(x, y, z)
$$

where $C_{i}^{\omega}$ are coefficients of the system of $m$ sufficiently complete basis functions $\chi_{i}^{\omega}$. Basis functions can be chosen from polynomials, radial basis functions, Bsplines, etc. and this selection will effect the accuracy, convergence, differential and computational properties of the approximate distance fields. Coefficients in eq. (2.58) can be computed by the least square method which minimizes the square of the difference between values of $\omega$ function and Euclidean distance map of sampled points distributed in the domain $[11,13]$ :

$$
F=\sum_{j=1}^{k}\left(\sum_{i=1}^{m} C_{i}^{\omega} \chi_{i}^{\omega}-d_{j}\right)^{2}
$$

where $k$ is the number of distance samples and $d_{j}$ is the $j^{\text {th }}$ signed distance measured from point $\left(x_{j}, y_{j}, z_{j}\right)$ to the closest boundary.

Later in a similar study the approximate distance field construction technique was further improved and the possibility of generation of such functions from CT (Computed Tomography) and MRI (Magnetic Resonance Imaging) images was investigated. It was demonstrated that by applying proper image processing methods, data from a greyscale CT or MRI image can be transferred into a binary image. 
Then Euclidean distances from every voxel/pixel in the binary image to the closest boundary voxel/pixel can be calculated by Saito's algorithm [57, 13]. Resulting distance map is piecewise and therefore not differentiable which makes it an unsuitable representation for a distance field function in meshfree method. However the constructed distance map can be used in a scheme such as eq. (2.59) to generate differentiable approximate distance fields and eventually provide a powerful tool for transferring image data into a distance field representation usable in a solution structure method.

The accuracy of approximate distance fields generated from images can be controlled by type, degree and resolution of basis functions as well as by the location and number of distance map samples [13]. 


\section{CHAPTER 3}

\section{HOMOGENIZED MECHANICAL PROPERTIES OF INHOMOGENEOUS MATERIAL}

After explaining the theoretical background on asymptotic homogenization method and meshfree solution structures, here in this chapter the details of our proposed hybrid platform will be investigated. Meshfree/asymptotic method uses a novel solution structure to solve asymptotic homogenization problem which provides the means for exact satisfaction of periodic boundary conditions, also this platform is empowered with a geometric block capable of acquiring realistic geometric models from complex microstructures.

We will start with the mathematical formulation of the solution in Section 3.1 and go over the possibility of development of a software platform to automatize the homogenization process, then in Section 3.2 performance of the hybrid meshfree/asymptotic method will be tested by comparing it to previously published data and/or test results gathered from available commercial softwares.

\subsection{MeshFree/Asymptotic Platform}

Going back to Section 2.1.1 we can recall that the homogenized mechanical properties of an inhomogeneous structure can be calculated by:

$$
E_{i j k l}^{H}=\frac{1}{|Y|} \int_{Y}\left(E_{i j k l}-E_{i j p m} \frac{\partial \chi_{p}^{k l}}{\partial y_{m}}\right) d Y
$$

Where $\boldsymbol{\chi}^{k l}(\boldsymbol{y})$ are Y-periodic functions which are the solution for weak form of balance equation:

$$
\int_{Y} E_{i j p m} \frac{\partial \chi_{p}^{k l}}{\partial y_{m}} \frac{\partial v_{i}}{\partial y_{j}} d Y=\int_{Y} E_{i j k l} \frac{\partial v_{i}}{\partial y_{j}} d Y \quad \forall v_{i} \in V
$$


In the weak formulation $\boldsymbol{v}(\boldsymbol{y})$ are also Y-periodic functions defined and smooth enough in domain $Y$. The most common method for solving eq. (3.2) is using FEA $[50,21]$ which involves generating a geometry conforming mesh on the boundary and enforcing periodic boundary conditions on boundary nodes and that requires pairing nodes on opposite faces of the RVE. In a general setting similarity of mesh distribution on paired boundaries of RVE cannot be guaranteed, specially when the geometry of inhomogeneities inside the RVE requires a non-periodic mesh or pores intersect with RVE sides.

To overcome this setback, in this dissertation a meshfree solution structure has been incorporated to solve eq. (3.2). This novel implementation of solution structure will eliminate any meshing and node pairing and also makes it possible to satisfy exact periodic boundary conditions.

\subsubsection{Solution structure for periodic boundary conditions}

According to Table 2.1, solution structure for a boundary value problem with nonhomogeneous Dirichlet boundary conditions can be represented by zero-order truncated series in the form of:

$$
u=\omega \Phi+\varphi
$$

Where $\omega$ is the distance field function representing the domain, $\Phi$ and $\varphi$ functions are designed in such way that they give the proposed solution structure enough degrees of freedom to satisfy governing equations and boundary conditions of the problem. $\omega \Phi$ satisfies the homogenous boundary conditions (recalling that the distance field functions are zero on the boundary), and consequently $u$ satisfies $\left.u\right|_{\partial Y}=\varphi$. Since $\Phi$ and $\varphi$ functions are usually unknown they could be approximated by linear com- 
bination of basis functions:

$$
\Phi=\sum_{i=1}^{n} C_{i}^{\Phi} \eta_{i} \quad, \quad \varphi=\sum_{i=1}^{n^{\prime}} C_{i}^{\varphi} \psi_{i}
$$

The solution structure eq. (3.3) does not pose any restrictions on the choices of basis functions $\left\{\eta_{i}, i=1, \ldots, n\right\}$ and also selection of these functions are completely independent of geometry of the domain. $\eta_{i}$ functions can be chosen from any common basis function system such as B-splines, polynomials, trigonometric polynomials, etc. On the other hand, $\left\{\psi_{i}, i=1, \ldots, n^{\prime}\right\}$ are supposed to generate the solution on the boundary and therefore they should satisfy periodicity conditions.

Substituting expansions eq. (3.4) back into eq. (3.3) leads to:

$$
u=\underbrace{\sum_{i=1}^{n} C_{i}^{\Phi}\left(\omega \eta_{i}\right)}_{1}+\underbrace{\sum_{i=1}^{n^{\prime}} C_{i}^{\varphi} \psi_{i}}_{2}
$$

In eq. (3.5) part (1) provides the approximation of function $u$ inside the domain and it satisfies the homogeneous Dirichlet boundary conditions and part (2) is the enrichment functions responsible for treatment of periodic boundary conditions. Going back to eq. (3.2) and using expression eq. (3.5) to form a solution for this equation results in:

$$
\chi_{m}^{k l}=\sum_{q=1}^{n+n^{\prime}} C_{q m}^{k l} \xi_{q} \quad, \quad \xi_{q}= \begin{cases}\omega \eta_{q} & q=1, \ldots, n \\ \psi_{q-n} & q=n+1, \ldots, n+n^{\prime}\end{cases}
$$

By applying Galerkin assumption which requires using the same group of functions for weight and test functions the modified form of weak formulation will be:

$$
\int_{Y} E_{i j m n} \frac{\partial \chi_{m}^{k l}}{\partial y_{n}} \frac{\partial \xi_{i}}{\partial y_{j}} d Y=\int_{Y} E_{i j k l} \frac{\partial \xi_{i}}{\partial y_{j}} d Y
$$

For calculation of all the members of elasticity tensor in a three dimensional domain eq. (3.7) should be solved for six different $k l$ indices to find out all necessary $\chi^{k l}$ 
functions $(k l=11,22,33,12 / 21,13 / 31,23 / 32)$. In two dimensional domains number of equations reduces to three and corresponding indices are $k l=11,22,12 / 21$.

Substituting series expansion of $\chi_{m}^{k l}$ functions into eq. (3.7) gives us:

$$
\int_{Y} E_{i j m n} \frac{\partial\left(\sum_{q=1}^{n+n^{\prime}} C_{q m}^{k l} \xi_{q}\right)}{\partial y_{n}} \frac{\partial \xi_{i}}{\partial y_{j}} d Y=\int_{Y} E_{i j k l} \frac{\partial \xi_{i}}{\partial y_{j}} d Y
$$

Taking the summation out of the integral and differentiating with respect to $C$ coefficients will result in a system of linear algebraic equations in the form of:

$$
[A][C]=[B]
$$

Where in fact for a certain $k l$, elements of matrix $[A]$ and vector $[B]$ are respectively calculated by:

$$
\begin{gathered}
a_{i j}=\int_{Y} E_{p q m n} \frac{\partial \xi_{i}}{\partial y_{n}} \frac{\partial \xi_{j}}{\partial y_{q}} d Y \\
b_{j}=\int_{Y} E_{p q k l} \frac{\partial \xi_{j}}{\partial y_{q}} d Y
\end{gathered}
$$

In the developed computational platform, to solve eq. (3.9) we will use basis functions that are constructed over Cartesian grid that does not conform to the shape of geometric model. This makes it possible to eliminate meshing (which could be very tedious and time consuming when images or CT scans are used as the geometry source) from the solution procedure. Assembly of the matrices and vectors in eq. (3.9) is performed by geometrically adaptive integration of eq. (3.10) and eq. (3.11), while automatic differentiation technique produces the numerical values of partial derivatives of basis functions. After solving eq. (3.9), values of $\left\{C_{q m}^{k l}\right\}$ coefficients are substituted back in eq. (3.6). Then by introducing $\left\{\chi_{m}^{k l}\right\}$ values from eq. (3.6) into eq. (3.1), homogenized elastic tensor for a given geometry can be calculated.

In order to give a more clear picture of solving homogenization problem with meshfree technique, here for a sample domain we will demonstrate the construction 
(a)

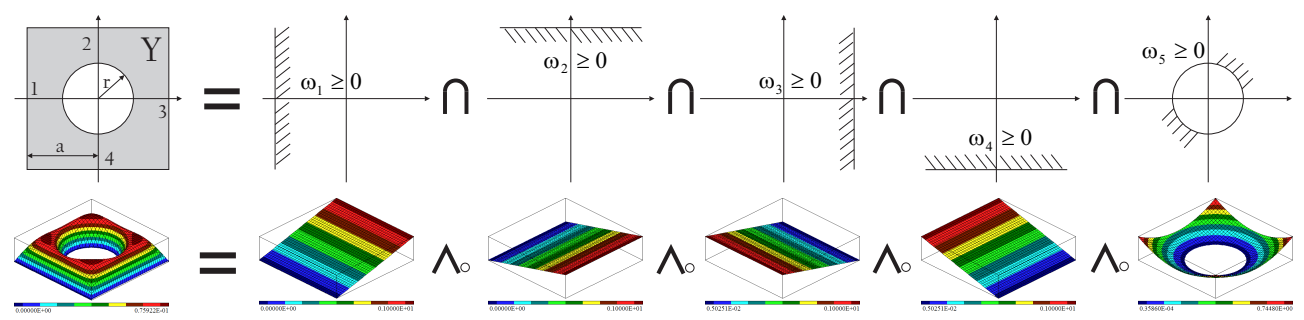

Figure 3.1: Construction of approximate distance fields using $R$-functions, (a) The native geometry is divided into five half-spaces, (b) Inequalities for $\omega_{1}$ to $\omega_{5}$ plotted as functions of $x$ and $y$ and the resulting $R_{0}$-conjunction $\omega$ has zero values and non-zero gradients on the boundaries and it is differentiable in the whole domain.

of different pieces involved in solution structure eq. (3.6) $(\omega, \eta$ and $\psi)$, and also we will show how coefficient matrix and load vector in system of linear algebraic equations in eq. (3.9) can be assembled.

Let us start with a geometric domain shown in Fig. 3.1, a rectangular RVE with a circular void. As shown the geometric domain can be represented as intersections and unions of the elementary half-spaces that can be defined by simple inequalities. In our case these half-spaces are: right side of edge 1 , left side of edge 3 , below edge 2, above edge 4 and outside of a disk of radius $r$. Each of these half-spaces is described by the following inequalities:

$$
\begin{array}{ll}
\omega_{1}(x, y)=x+a \geq 0 & \omega_{2}(x, y)=a-y \geq 0 \\
\omega_{3}(x, y)=a-x \geq 0 & \omega_{4}(x, y)=y+a \geq 0 \\
\omega_{5}(x, y)=\frac{1}{2 r}\left(x^{2}+y^{2}-r^{2}\right) \geq 0 &
\end{array}
$$

Therefore the boolean expression for domain $Y$ could be presented as:

$$
Y=\left(\omega_{1} \geq 0\right) \cap\left(\omega_{2} \geq 0\right) \cap\left(\omega_{3} \geq 0\right) \cap\left(\omega_{4} \geq 0\right) \cap\left(\omega_{5} \geq 0\right)
$$

In theory of $R$-functions to construct a real-valued function $\omega$ which could represent domain $Y$, the boolean operators need to be replaced with corresponding $R_{0^{-}}$ function, therefore the final expression for $\omega$ which is positive inside the geometric 


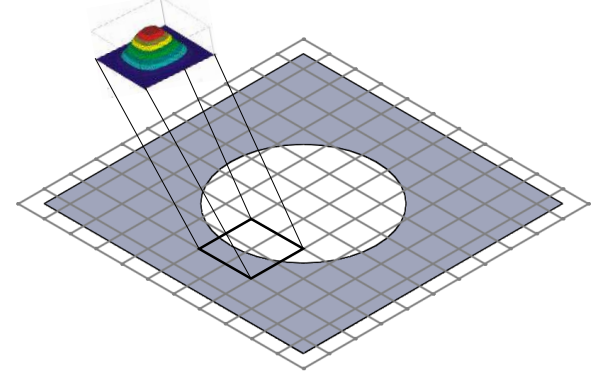

Figure 3.2: Basis functions created over a Cartesian grid on the domain.

domain $Y$, negative outside, and vanishes on its boundary will be:

$$
\omega=\omega_{1} \wedge_{0} \omega_{2} \wedge_{0} \omega_{3} \wedge_{0} \omega_{4} \wedge_{0} \omega_{5}
$$

Next step for completion of our solution structure is to choose the system of basis functions $\eta$, as mentioned before any of common basis function systems can be an acceptable choice since the solution structure poses no restriction on basis functions. Fig. 3.2 shows a system of polynomial basis functions constructed over a non-conforming grid on the domain.

After explaining the construction of $\omega$ and $\eta$, the only remaining element of solution structure is $\psi$ functions which are responsible for treatment of periodic boundary conditions and therefore they should satisfy periodicity requirements. In other words $\psi$ functions which here we call the Enrichment Functions have to be constructed in such way that they have the same behavior on opposing sides of the RVE. In this dissertation, enrichment functions for two dimensional domains are always generated in pairs and therefore the total number of them $\left(n^{\prime}\right)$ is always an even number. In each pair one function is responsible for reconstruction of boundary values on boundaries parallel to $x$ axis and the other one does the same for boundaries parallel to $y$ axis. If we assume that for a rectangular RVE in $x y$ 

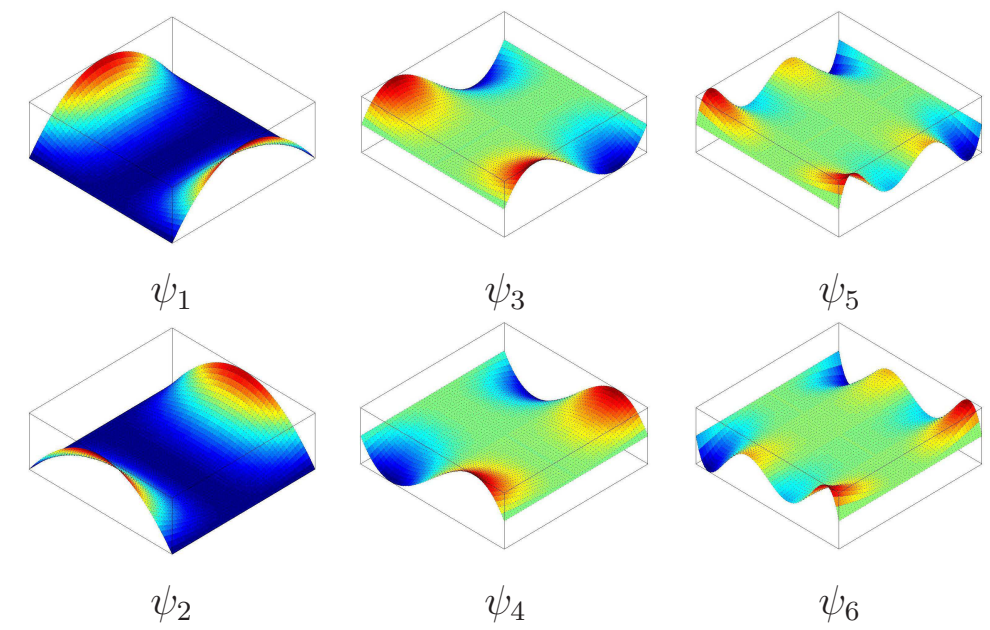

Figure 3.3: Enrichment functions created in pairs via eq. (3.15) for $n^{\prime}=6$.

plane defined by its corners ${ }^{1}$ the total of number of enrichment functions is $n^{\prime}=2 k$, then the $k$-th pair of enrichment functions can be formulated as:

$$
\begin{aligned}
\Delta x & =x_{\max }-x_{\min } \quad \Delta y=y_{\max }-y_{\min } \\
x_{c} & =\frac{x_{\max }+x_{\min }}{2} \quad y_{c}=\frac{y_{\max }+y_{\min }}{2} \\
\text { For } k=1 \quad & \psi_{1}=\left[\left(\frac{\Delta x}{2}\right)^{2}-\left(x-x_{c}\right)^{2}\right]\left(y-y_{c}\right)^{2} \\
\psi_{2} & =\left[\left(\frac{\Delta y}{2}\right)^{2}-\left(y-y_{c}\right)^{2}\right]\left(x-x_{c}\right)^{2} \\
\text { For } k=2,3, \ldots \quad \psi_{2 k-1} & =\left(y-y_{c}\right)^{2} \prod_{j=0}^{2^{k}}\left[x-\left(x_{\min }+\frac{j}{2^{k}} \Delta x\right)\right] \\
\psi_{2 k} & =\left(x-x_{c}\right)^{2} \prod_{j=0}^{2^{k}}\left[y-\left(y_{\min }+\frac{j}{2^{k}} \Delta y\right)\right]
\end{aligned}
$$

In Fig. 3.3 a group of enrichment functions constructed by eq. (3.15) for $n^{\prime}=6$ are demonstrated and it is clearly shown that how these functions satisfy periodicity conditions.

\footnotetext{
${ }^{1}\left(x_{\min }, y_{\min }\right),\left(x_{\max }, y_{\min }\right),\left(x_{\max }, y_{\max }\right)$ and $\left(x_{\min }, y_{\max }\right)$.
} 


\begin{tabular}{|c|c|c|c|c|c|c|c|c|c|c|c|c|c|}
\hline$\xi_{1}$ & $\frac{\partial \xi_{1}}{\partial x}$ & $\frac{\partial \xi_{1}}{\partial y}$ & $\ldots$ & $\xi_{n}$ & $\frac{\partial \xi_{n}}{\partial x}$ & $\frac{\partial \xi_{n}}{\partial y}$ & $\xi_{n+1}$ & $\frac{\partial \xi_{n+1}}{\partial x}$ & $\frac{\partial \xi_{n+1}}{\partial y}$ & $\ldots$ & $\xi_{n+n^{\prime}}$ & $\frac{\partial \xi_{n+n^{\prime}}}{\partial x}$ & $\frac{\partial \xi_{n+n^{\prime}}}{\partial y}$ \\
\hline \multicolumn{7}{|c|}{ Block 0} & \multicolumn{3}{|c|}{ Block 1} & & \multicolumn{3}{|c|}{ Block n } \\
\hline
\end{tabular}

Figure 3.4: Schematic for a tuple sequence structure.

Now by having all the necessary information on functions involved in solution structure, $\chi$ functions can be constructed in the form of eq. (3.6). In the developed computational platform, at any calculation point values of each $\xi_{q}$ function and its derivatives are stored in a differential tuple (see Section 2.2.3 for information on tuple construction). Then these tuples are assembled one after each other and form a tuple sequence which can provide run-time access to values and derivative of test functions in eqs. (3.10) and (3.11). Fig. 3.4 shows the schematic for a tuple sequence structure.

As mentioned earlier assembly of coefficient matrix and load vector in eq. (3.9) is done by geometrically adaptive integration, which like any other numerical integration method requires a proper distribution of integration points in the domain to numerically evaluate the integrants at those points. Here in our computational platform, the latter is done by creating a nonconforming geometric grid over the domain and using the grid cells as a pattern for distribution of Gauss integration points. The tuple sequence structure gets evaluated at every single integration point and the numerical values of derivatives stored in the tuples will then be used for integration purposes. The mentioned grid and distribution of Gauss points for the RVE under discussion are respectively shown in Figs. 3.5(a) and (b).

Number of the grid lines and Gauss integration points density in each grid can be adjusted based on the complexity of geometry in a manner that provides enough resolution to perform numerical integration. For example, if a finer resolution near the boundaries is required to make sure that all the geometric features are being 


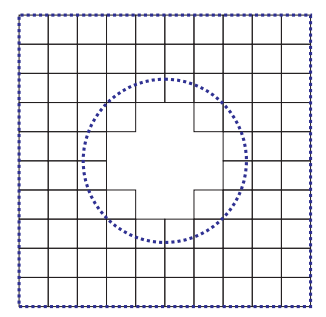

(a)

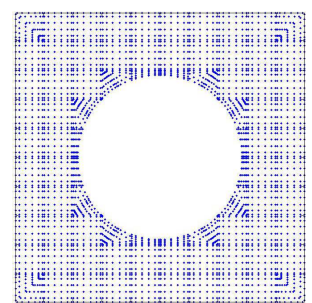

(b)

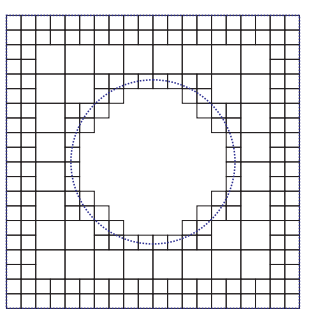

(c)

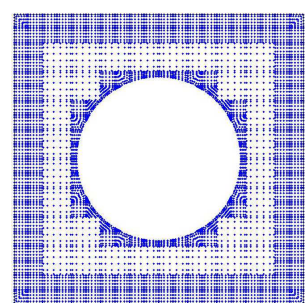

(d)

Figure 3.5: (a) Nonconforming uniform grid over the domain, (b) Distribution of Gauss integration points in the uniform grid cells, (c) Nonconforming grid with refined boundary cells, (d) Distribution of Gauss integration points in the refined grid cells.

captured, the developed computational platform can achieve that by creating a finer grid close to the boundaries and eventually having a higher density of integration points in the boundary regions, Figs. 3.5(c) and (d) show an example of grid refinement close to the boundaries.

By having the Gauss points distribution in the domain, numerical values for the elements in coefficient matrix and load vector of system of linear algebraic equations in eq. (3.9) can be calculated and the system could be solved for unknown $\left\{C_{q m}^{k l}\right\}$ which eventually leads to finding the homogenized mechanical properties of the domain.

\subsubsection{Software prototype work flow}

As a result of this dissertation the mathematical concept of computational platform described in Section 3.1.1 has been turned into a $\mathrm{C}++$ computer program to show the possibility of development of a software package working based on the hybrid meshfree/asymptotic idea and also testing its performance in various numerical experiments. 
Work flow of the mentioned platform is presented as a block diagram in Fig. 3.6. As shown, the platform is formed by interconnecting four independent blocks. The first component is the User Interface block which provides the connection between the user and the solver. By accessing this block, user can import the desired 2D image as source of the native geometry, choose type of the basis functions based on the complexity of the geometry and select the proper homogenization method according to the physical property which has to be homogenized. Each of these inputs are connected to one of the three blocks in the solver. Data from microstructure image is transferred to the Geometric Block and after being processed, a binary image of each domain in the microstructure is rendered which eventually leads to the generation of distance field functions. Homogenization Block provides the geometry of the RVE, necessary boundary conditions and valid governing equations at micro scale level. It also gives us the proper recipe for calculation of homogenized properties.

In the center of solver lies the MeshFree Block. Distance field representation of the geometry, governing equations, related boundary conditions and also type of the basis functions are provided as inputs for it and inside this block a solution structure is formed in such way that satisfies the exact boundary conditions. Then the generated structure in conjunction with an automatic differentiation toolbox is used to produce the terms in micro level balance equation and by applying adaptive integration, a system of linear equations is created which its solution will provide the necessary information to evaluate the homogenized properties of the given microstructure.

The connection lines linking User Interface with Geometric Block and MeshFree Block provides a number of user control parameters which can be adjusted based on the complexity of the geometry and required accuracy (see Fig. 3.7). Going 


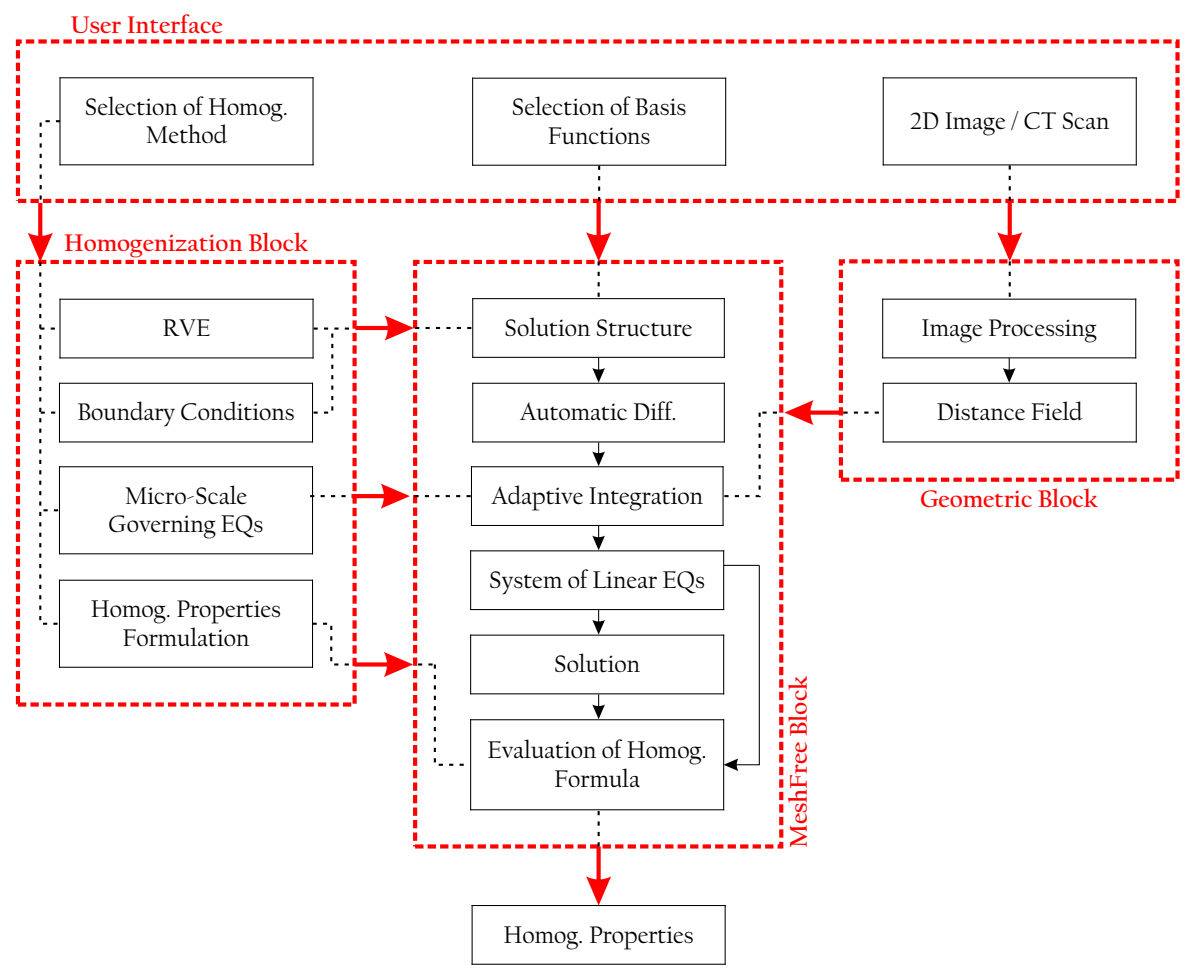

Figure 3.6: Block diagram for Meshfree/Asymptotic homogenization platform.

from user interface to geometric block, user can choose type and degree of the basis functions used to create distance fields and in case of reading geometry from two dimensional images number of distance samples also can be adjusted. The connection to the meshfree block gives the user the ability to specify number of the grids over the domain, refinement degree of the grids, number of Gauss points in each grid cell, resolution for small feature detection and number of enrichment functions.

One of the very important advantages of the developed platform is that the pieces connecting to the MeshFree block function independently and they can be replaced by any custom made toolbox as long as the replacement is capable of generating the required outputs, which makes the platform compatible with various types of problems. 


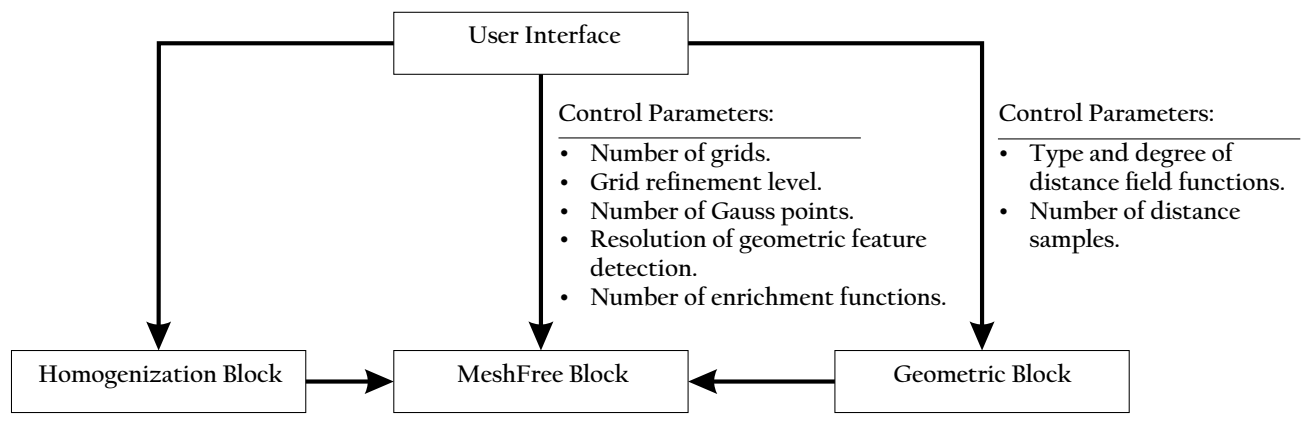

Figure 3.7: Control parameters accessible by user in the Meshfree/Asymptotic homogenization platform.

\subsection{Numerical Experiments}

In this section performance of the developed computational platform is tested in comparison to previously published data or results of commercially available software packages. Numerical experiments presented here, based on their geometry are divided into three different categories: geometries presented in previously published literature, geometries resulting in fully anisotropic elasticity tensor and geometries captured from two dimensional micrograph images. In each category number of examples are presented ad results are compared with a proper validation criterion.

\subsubsection{Geometries mentioned in literature}

\section{Carbon/Epoxy composite}

For the first example, a RVE with a rather simple geometry is selected. Since this sample was previously studied in [40], results of Meshfree/Asymptotic method could be compared to conventional finite element method. The mentioned RVE consists of a composite with unidirectional carbon fibers embedded in epoxy.

In [40] such combination was investigated by assuming a square RVE with a circular inclusion as shown in Fig. 3.8 and the homogenized properties were calculated 


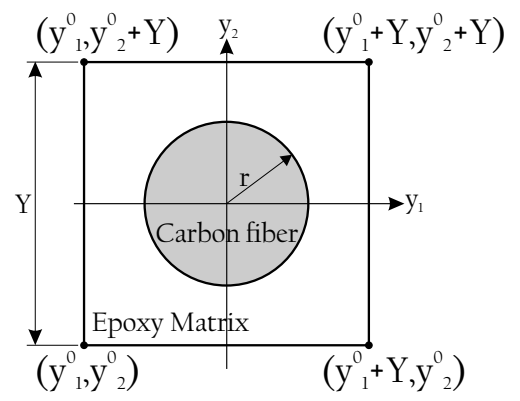

Figure 3.8: RVE for a composite with periodic circular inclusions $\left(V_{f}=0.25\right)$.

for different volume fractions $\left(V_{f}\right)$ of the fiber. Following data are used for material properties alongside with plane strain assumption:

$$
\begin{array}{lll}
\text { Carbon: } & E_{1}=15 \text { GPa } & \nu_{1}=0.20 \\
\text { Epoxy: } & E_{2}=3 \text { GPa } & \nu_{2}=0.35
\end{array}
$$

As presented in Fig. 3.8 the geometry of RVE for this numerical experiment is very similar to Fig. 3.1, the only difference is the replacement of circular void with carbon fibers.

In our homogenization platform the structure of RVE is imported into the geometric engine and distance field functions, nonconforming grid and Gauss integration points distribution are prepared for epoxy matrix and carbon fibers separately. Figs. 3.9(a)-(d) respectively show these parameters. Then a solution structure same as eq. (3.6) is formed with four enrichment functions which are customized for a square domain with unit length sides:

$$
\begin{aligned}
& \psi_{1}=\left(0.5^{2}-(x-0.5)^{2}\right)(y-0.5)^{2} \quad \psi_{3}=x(x-0.5)(x-1)(y-0.5)^{2} \\
& \psi_{2}=\left(0.5^{2}-(y-0.5)^{2}\right)(x-0.5)^{2} \quad \psi_{4}=y(y-0.5)(y-1)(x-0.5)^{2}
\end{aligned}
$$

These functions satisfy the periodic boundary conditions in a $Y$-periodic domain:

$$
\chi\left(y_{1}, y_{2}\right)=\chi\left(y_{1}+Y, y_{2}\right)=\chi\left(y_{1}, y_{2}+Y\right)=\chi\left(y_{1}+Y, y_{2}+Y\right)
$$

For a $2 \mathrm{D}$ problem three sets of $\boldsymbol{\chi}$ functions are required $(k l=11,12 / 21,22)$ to fully 


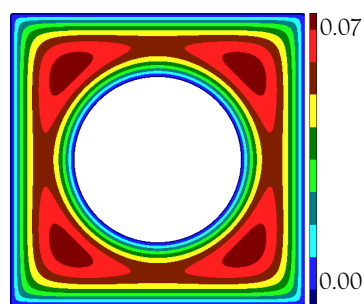

(a)

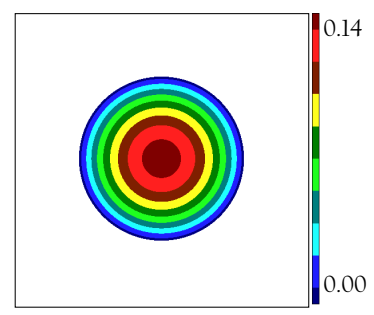

(b)

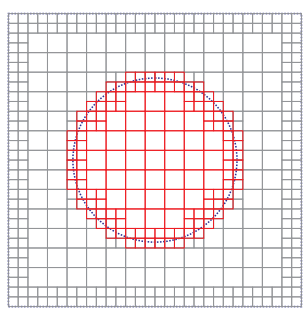

(c)

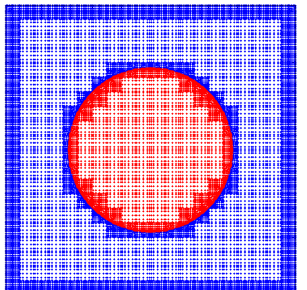

(d)

Figure 3.9: Geometry modeling (a) Distance field functions for epoxy; (b) Distance field functions for carbon fiber; (c) Nonconforming grid over the domain; (d) Gauss integration points distribution.

form the homogenized elasticity tensor by using equation eq. (3.1). Fig. 3.10 shows the $\chi$ functions for this particular problem which are consisted of trigonometricpolynomial distance functions and a set of four polynomial enrichment functions. Homogenization process is performed for different volume fractions of fiber and results are compared with [40] and also with analytical values derived for upper and lower bounds of homogenized properties by Hashin and Shtrikman [18, 19]. Fig. 3.11 shows the homogenized properties obtained from MeshFree/Asymptotic platform as functions of fiber volume fraction and compares them with finite element results. Calculated properties are perfectly consistent with Hashin-Shtrikman upper and lower bounds and also in a very good agreement, within $1 \%$ of the results of finite elements.

\section{Square RVE with a square void}

In a study similar to the previous example homogenized properties of a square RVE with a square shape void was investigated in [4]. RVE was modeled similar to Fig. 3.12 , and it was assumed that the structure is in plane stress and material properties are $E=87 G P a$ and $\nu=0.22$. Homogenized properties of the RVE were calculated as a function of material volume fraction by using conventional finite elements. 

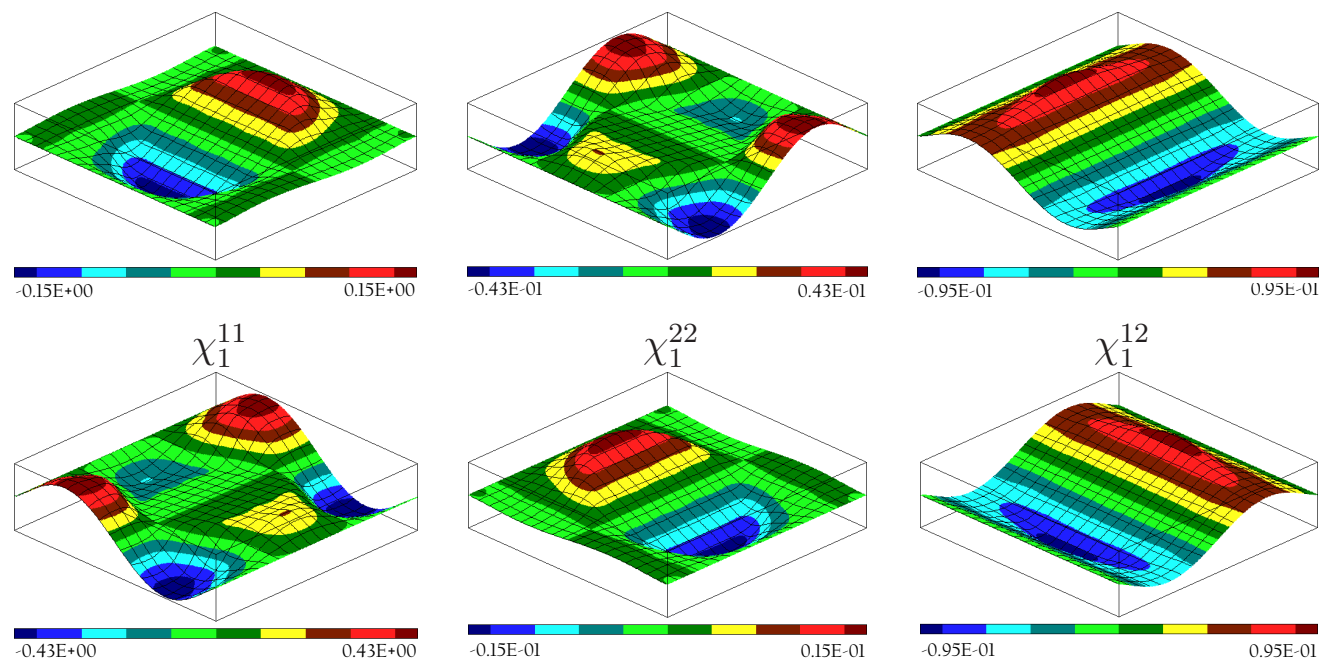

$\chi_{2}^{11}$

$\chi_{2}^{22}$

$\chi_{2}^{12}$

Figure 3.10: Basis $\left\{\chi_{m}^{k l}\right\}$ functions for RVE with circular inclusions.

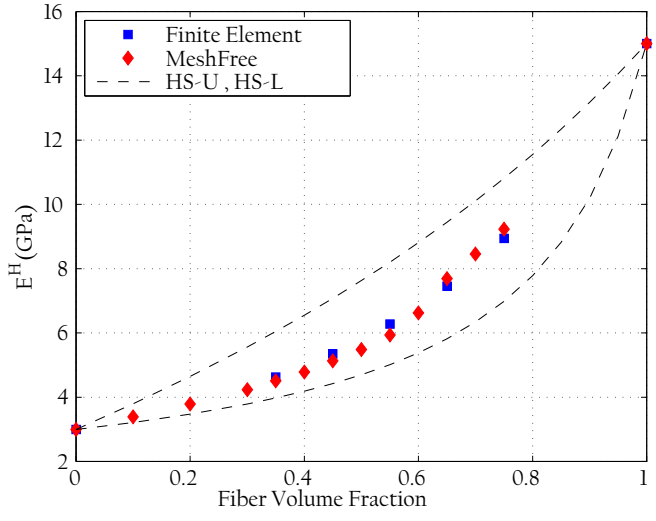

(a)

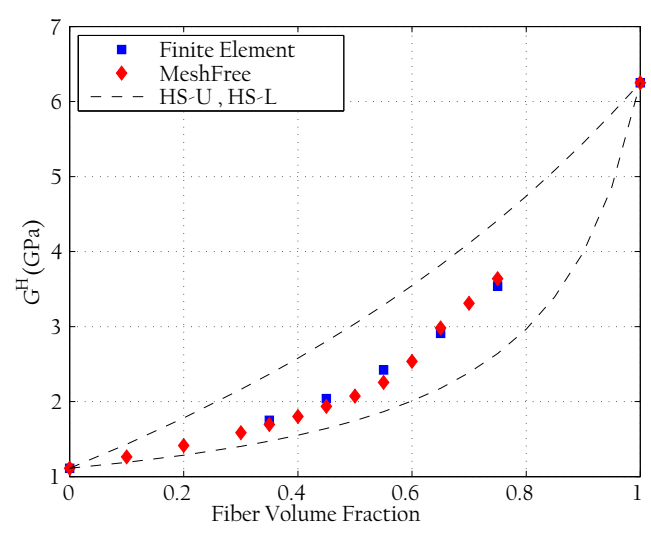

(b)

Figure 3.11: Homogenized (a) Young's and (b) Shear modulus for a square RVE with a circular fiber computed with MeshFree method and compared with finite elements results in [40] and Hashin-Shtrikman upper and lower bounds. 


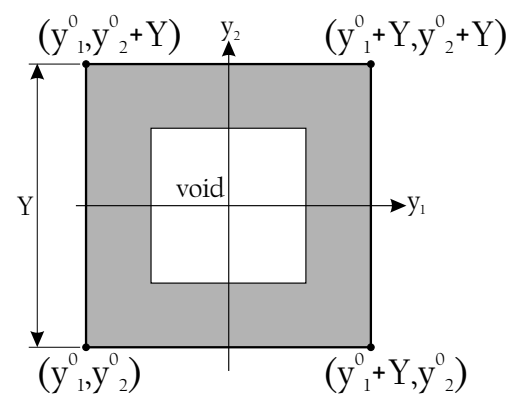

Figure 3.12: RVE for a square domain with a square void $\left(V_{f}=0.70\right)$.

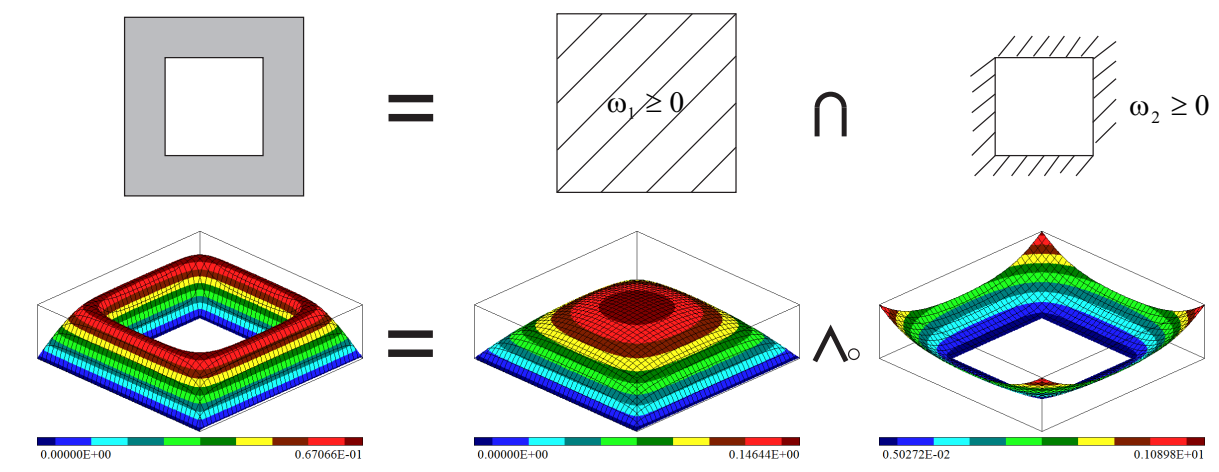

Figure 3.13: Construction of approximate distance field functions for a RVE with a square void using $R_{0}$-conjunction.

Here the same RVE is studied by meshfree/asymptotic platform and results are compared to the ones published in [4]. To do so, first the geometry is broken done to primitive shapes which are two squares and then by using $R_{0}$-functions the distance field representation of the structure is prepared. Then nonconforming grid is generated over the domain and Gauss integration points are distributed. A solution structure similar to eq. (3.6) with the same enrichment functions as eq. (3.17) is formed and eventually $\chi$ functions are calculated. Fig. 3.13 shows construction of distance field functions for the RVE, the nonconforming grid and Gauss points are presented in Fig. 3.14 and $\chi$ functions are shown in Fig. 3.15.

The above procedure is repeated for different volume fractions of material and homogenized properties of the RVE are calculated and stored. Fig. 3.16 shows 


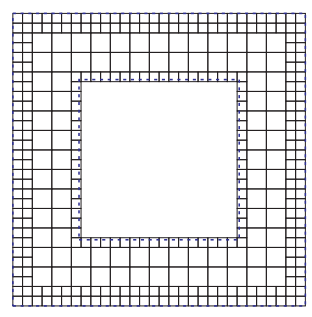

(a)

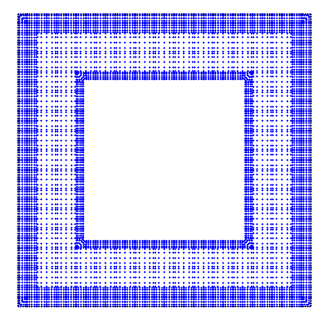

(b)

Figure 3.14: (a) Nonconforming grid over the RVE; (b) Distribution of Gauss integration points.
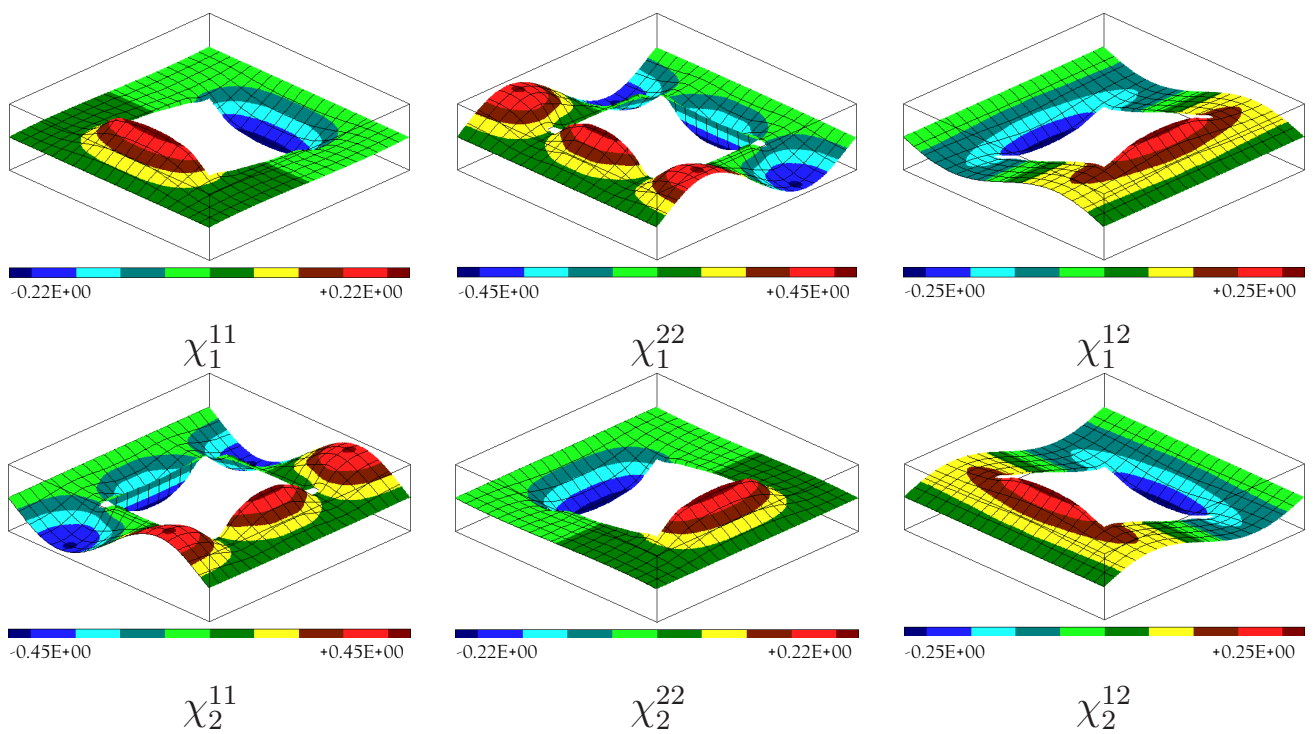

Figure 3.15: Basis $\left\{\chi_{m}^{k l}\right\}$ functions for RVE with a square void and $v_{f}=0.70$. 


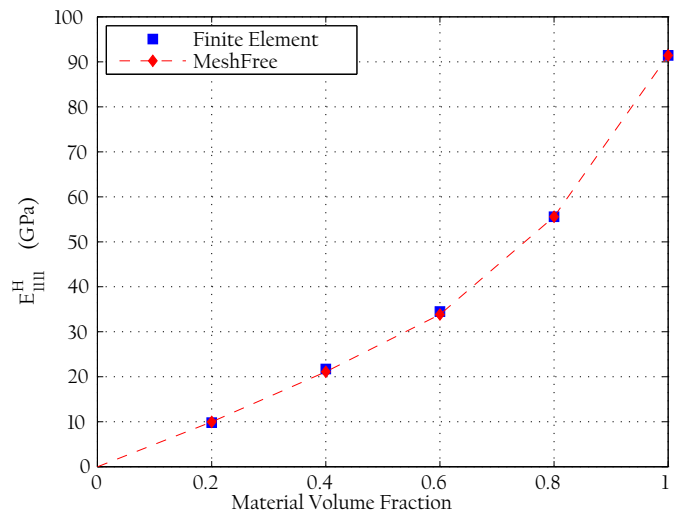

(a)

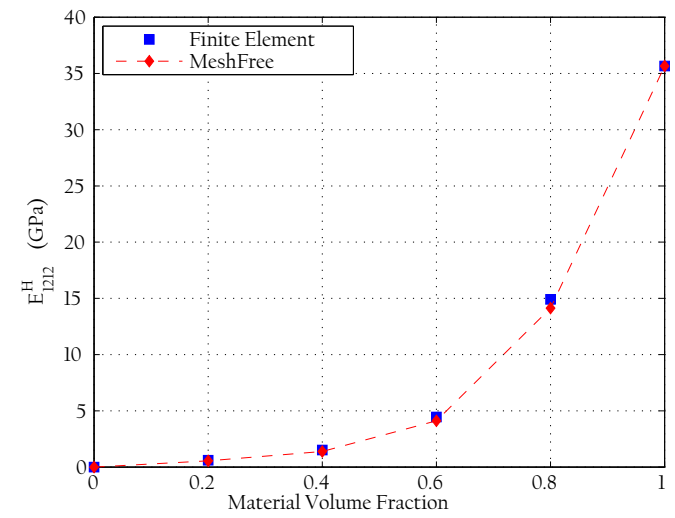

(b)

Figure 3.16: Elements of homogenized elasticity tensor (a) $E_{1111}^{H}$ (b) $E_{1212}^{H}$ as functions of material volume fraction, computed with meshfree and compared to finite element results published in [4].

the elements of the homogenized elasticity tensor presented as functions of material volume fraction and the outcome of meshfree/asymptotic method and conventional finite elements published in [4] are very close, with the maximum difference of $9 \%$.

\section{Periodic masonry structure}

Masonry is a two-phase material comprised of brick and mortar joints, normally arranged periodically and studying its in-plane load deformation characteristic is very important for designing and retrofitting masonry structures. Instead of applying expensive and time consuming laboratory and field experiments, in [69] an alternative numerical method was proposed for calculation of effective material properties of such structures. The authors combined an equivalent periodic eigenstrain method with a strain energy approach to formulate the effective stiffness of the masonry.

Here same geometries used in [69] are studied with meshfree/asymptotic platform and results are compared to the published data. The RVE structure for a masonry block is shown in Fig. 3.17 and following material and geometric properties along 


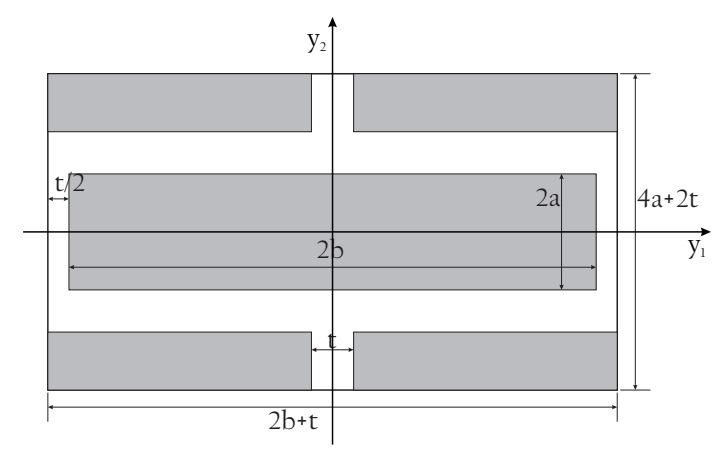

Figure 3.17: RVE for a masonry block with mortar thickness $t=20 \mathrm{~mm}$.

with plane stress assumption are used in the modeling process:

$$
\begin{array}{lll}
\text { Brick: } & E_{b}=11 \mathrm{GPa} & \nu_{b}=0.20 \\
& 2 a=55 \mathrm{~mm} & 2 b=250 \mathrm{~mm} \\
\text { Mortar: } & E_{m}=2.2 \mathrm{GPa} & \nu_{m}=0.25
\end{array}
$$

Following the steps in meshfree/asymptotic method, first distance field functions for each phase in the RVE is constructed, nonconforming grid is formed over the geometry and Gauss integration points are distributed. Figs. 3.18(a)-(d) respectively show the distance field functions for brick, mortar, grid and Gauss points.

Solution structure for periodic boundary conditions in eq. (3.6) with the same enrichment functions as eq. (3.17) is used to create the series expansion for $\chi$ functions, Fig. 3.19 shows these functions. Eventually homogenized elasticity tensor is computed and same process is repeated for different values of $t$ and results are plotted in Fig. 3.20 against the data presented in [69]. Comparing the results of meshfree/asymptotic and eigenstrain method shows that these two are in a very good agreement with each other (within $11 \%$ of each other) and the developed platform is performing with an acceptable accuracy. 


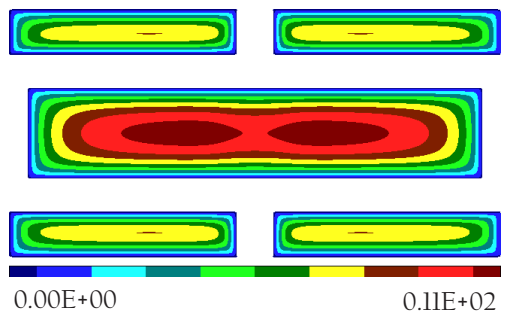

(a)

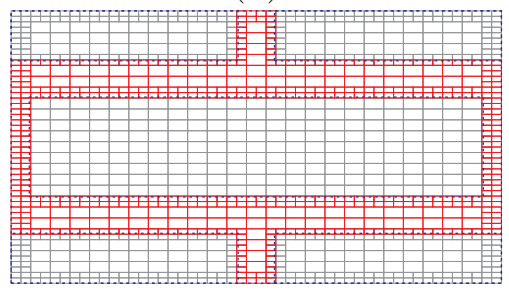

(c)

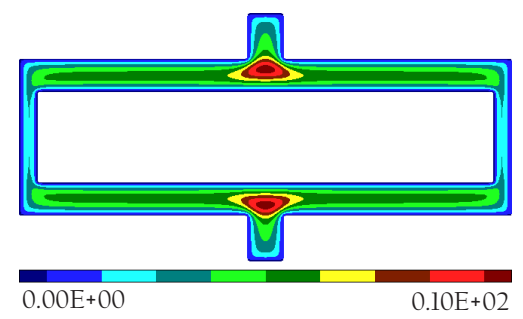

(b)

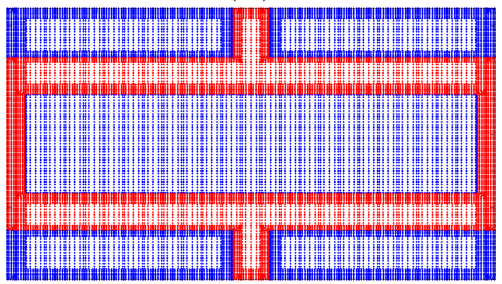

(d)

Figure 3.18: Geometry modeling for a masonry block with $t=20 \mathrm{~mm}$ (a) Distance field functions for brick; (b) Distance field functions for mortar; (c) Nonconforming grid over the domain; (d) Gauss integration points distribution.

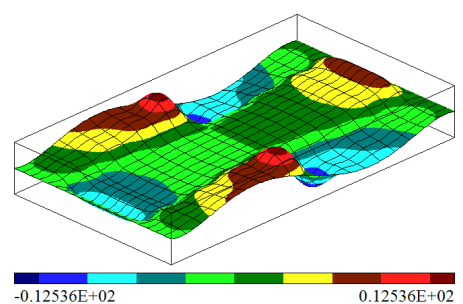

$\chi_{1}^{11}$

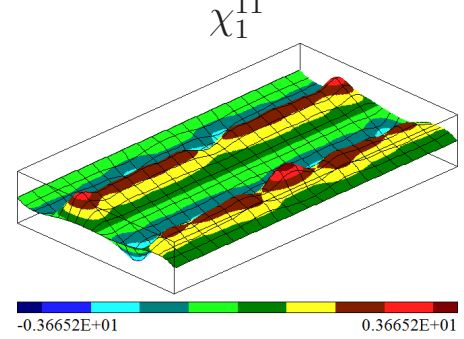

$\chi_{2}^{11}$

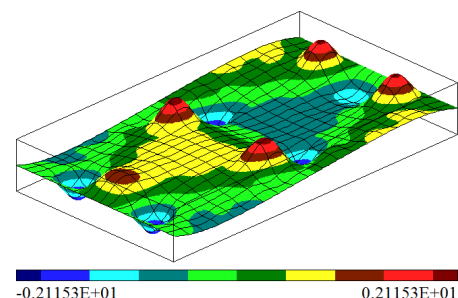

$\chi_{1}^{22}$

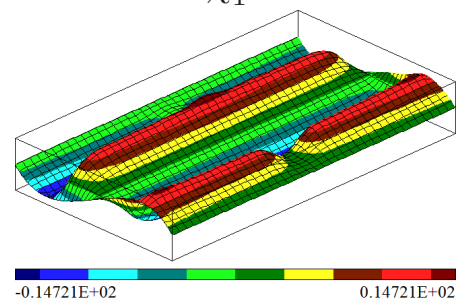

$\chi_{2}^{22}$

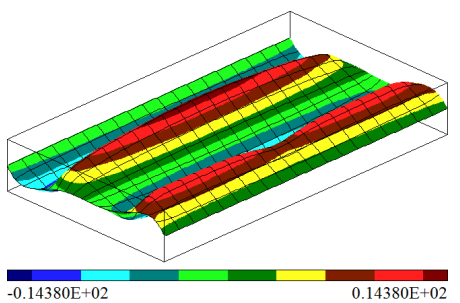

$\chi_{1}^{12}$

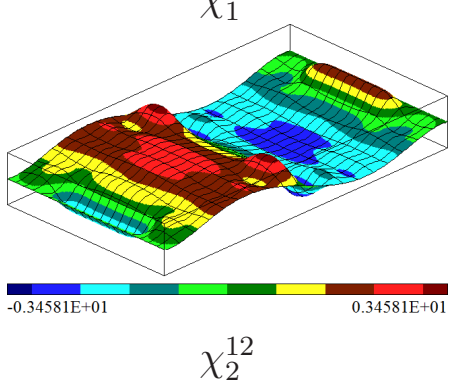

Figure 3.19: Basis $\left\{\chi_{m}^{k l}\right\}$ functions for a masonry RVE with $t=20 \mathrm{~mm}$. 


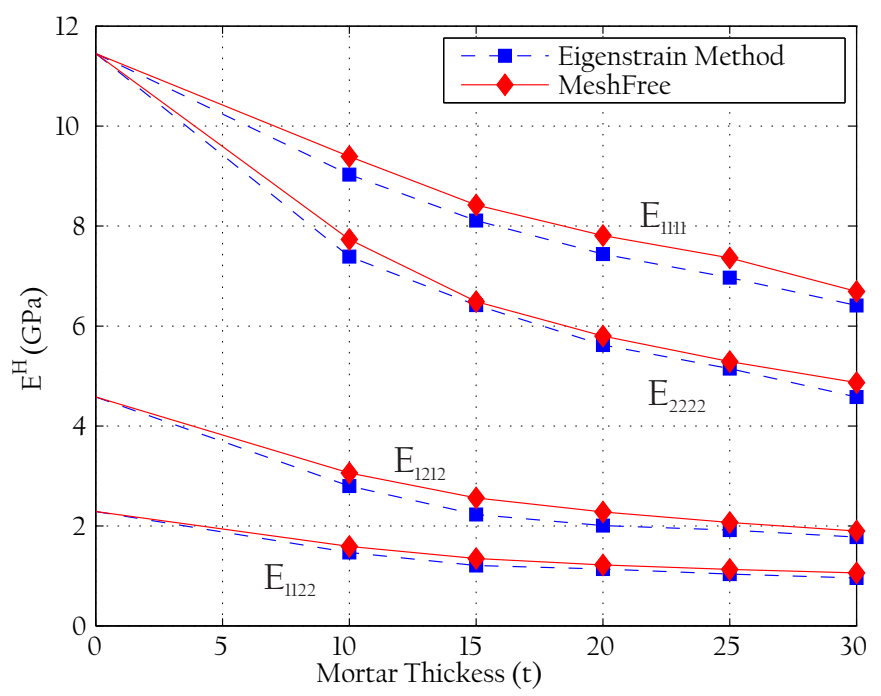

Figure 3.20: Elements of homogenized elasticity tensor computed with meshfree/asymptotic and results of eigenstrain method published in [69].

\subsubsection{Fully anisotropic geometries}

All the geometries discussed in Section 3.2.1 have some kind of symmetry and because of that the resulting homogenized elasticity tensor is either isotropic or orthotropic. These geometries will never lead to fully anisotropic results and always $E_{1112}^{H}=E_{1211}^{H}=E_{2212}^{H}=E_{1222}^{H}=0$. Numerical experiments in this section is chosen in such way that they will lead to fully anisotropic material behavior, therefore we can test the capability of the developed platform in handling nonsymmetric structures.

\section{Perforated plate}

In the first nonsymmetric numerical example a plate with periodic structure of elliptical voids is studied and its homogenized stiffness matrix is calculated by using meshfree/asymptotic platform, Fig. 3.21(a) shows the RVE configuration. It is 


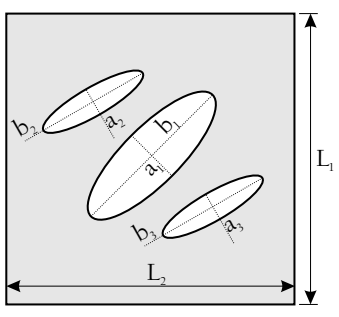

(a)

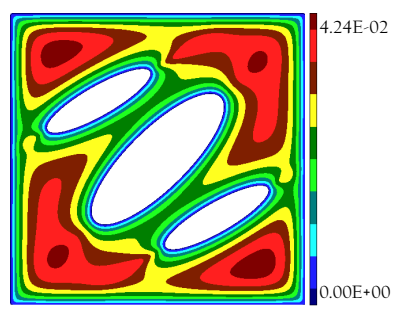

(b)

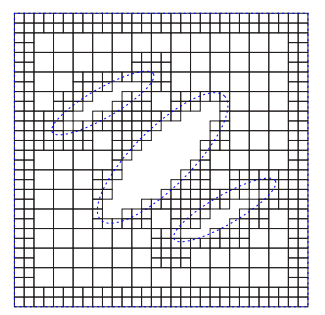

(c)

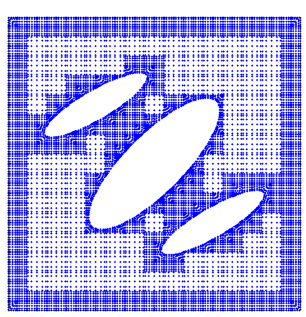

(d)

Figure 3.21: Geometry modeling for a perforated plate (a) RVE; (b) Distance field functions; (c) Nonconforming grid over the domain; (d) Gauss integration points distribution.

assumed that RVE is in plain strain and $\frac{L_{1}}{L_{2}}=1, \frac{a_{1}}{b_{1}}=\frac{1}{3}, \frac{a_{2}}{b_{2}}=\frac{a_{3}}{b_{3}}=\frac{1}{3}, E=$ 26.67 GPa and $\nu=0.33$.

Same as before distance field functions, nonconforming grid, Gauss integration point distribution and solution structure are prepared for the RVE. Fig. 3.21(b)(d) shows the components involved in geometry modeling. By having the proper geometric model for a meshfree platform, the coefficient matrix and load vector in the system of liner algebraic equation are assembled, $\chi$ functions and consequently homogenized elasticity tensor are computed. Resulting $\chi$ functions are presented in Fig. 3.22.

To validate the outputs of meshfree/asymptotic platform, a strain energy based scheme is used to predict effective material properties. In Section 2.1.3 details of strain energy method are discussed and it is explained how Hill condition is the necessary and sufficient condition for the energetically and mechanically defined properties to be to equal to each other and how applying certain types of boundary condition on a RVE can satisfy the requirements of Hill principle.

In the energy method used in this study, the RVE is modeled as a two dimensional geometry in COMSOL and three sets of kinematic uniform boundary conditions (KUBC) are applied to the boundaries of RVE. Stress distribution and strain 

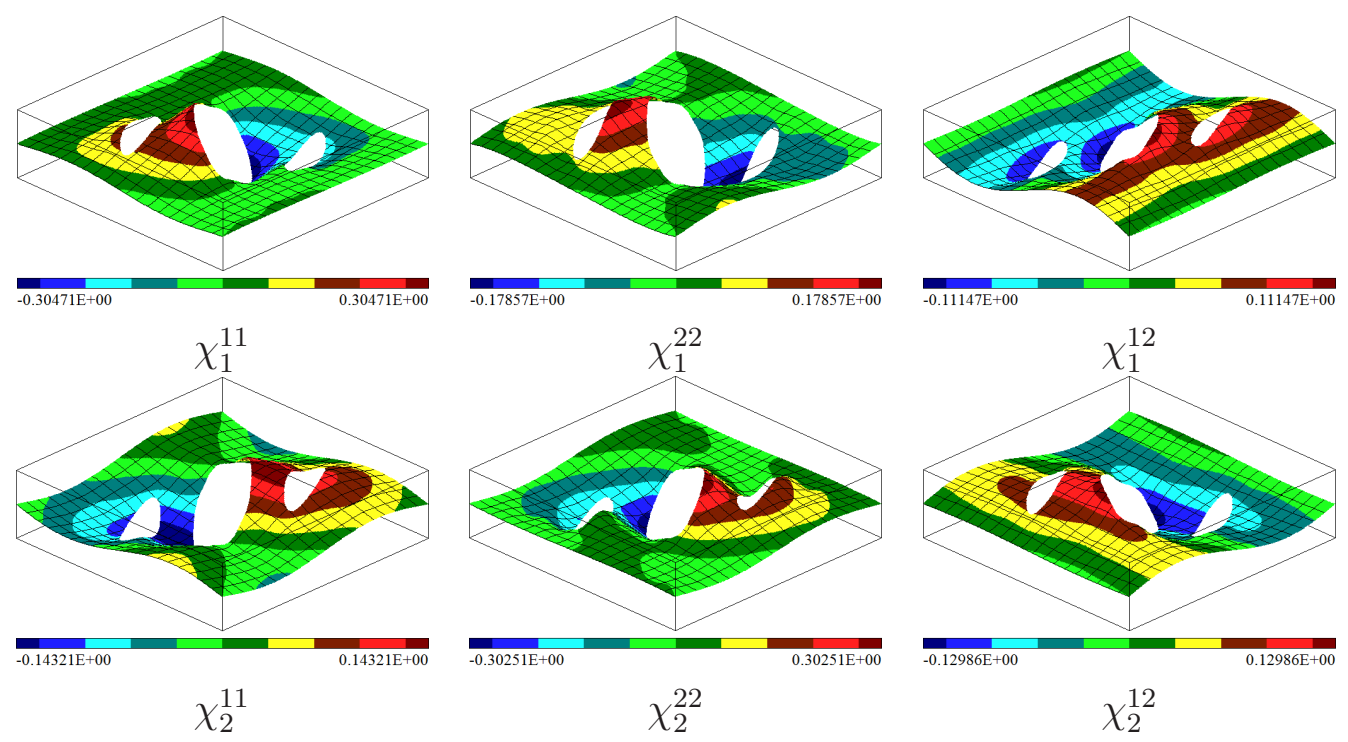

Figure 3.22: Basis $\left\{\chi_{m}^{k l}\right\}$ functions for a perforated plate.

energies are measured by using conventional finite elements and results obtained from each set of boundary conditions are used to calculate the elements in one of the columns of the elasticity tensor. Fig. 3.23 shows the model and corresponding mesh created in COMSOL and different set of boundary conditions applied to the RVE edges.

Elements of homogenized stiffness matrix $\left[E^{H}\right]$ obtained from meshfree/asymptotic and strain energy method are both recorded in Table 3.1 and the corresponding difference for each element is calculated.

\subsubsection{Geometries captured from images}

So far all the geometries of RVEs used in our numerical experiments were modeled by creating primitive geometric shapes and combining them with each other through theory of $R$-functions. In this section we are going to demonstrate one of the significant advantages of meshfree/asymptotic platform which is the capability 
KUBC
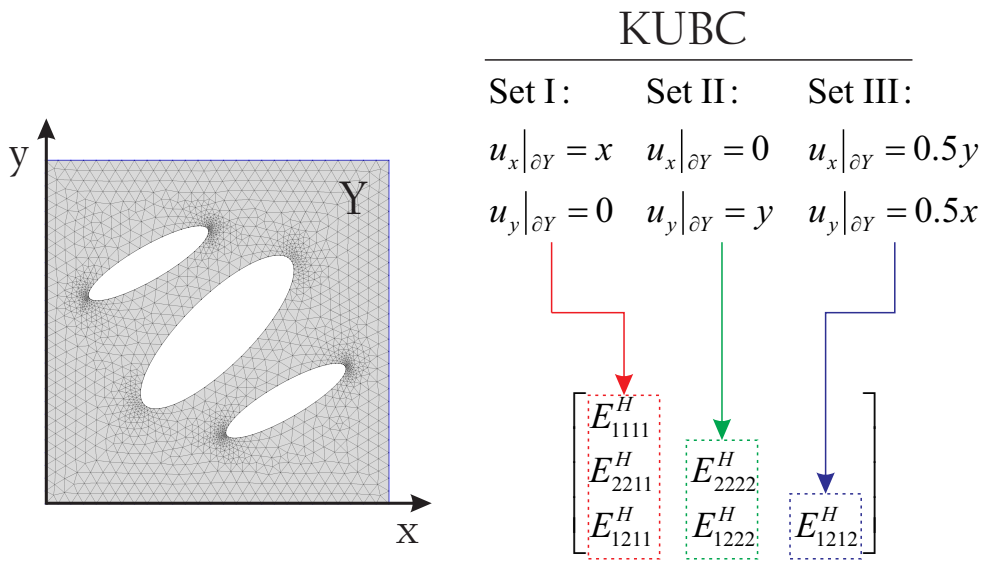

Figure 3.23: COMSOL model of the RVE for perforated plate, KUBC boundary conditions and elements of homogenized elasticity tensor corresponding to each set of boundary conditions.

Table 3.1: Homogenized stiffness matrix of the RVE for perforated plate.

\begin{tabular}{lccc}
\hline & Strain Energy Method & MeshFree/Asymptotic & Difference \\
\hline$E_{1111}^{H}$ & 23.35 & 23.14 & $0.90 \%$ \\
$E_{2222}^{H}$ & 19.86 & 20.76 & $4.53 \%$ \\
$E_{1212}^{H}$ & 7.06 & 6.60 & $6.52 \%$ \\
$E_{2211}^{H}$ & 9.41 & 9.40 & $0.1 \%$ \\
$E_{1211}^{H}$ & 2.28 & 2.42 & $6.14 \%$ \\
$E_{1222}^{H}$ & 1.77 & 1.92 & $8.47 \%$ \\
\hline
\end{tabular}




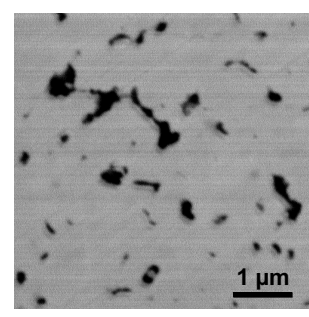

(a)

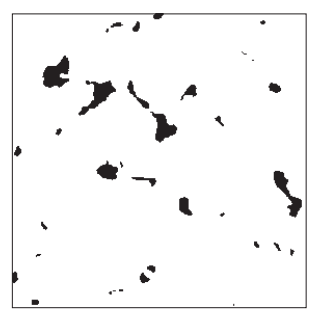

(b)

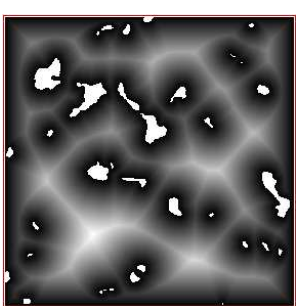

(c)

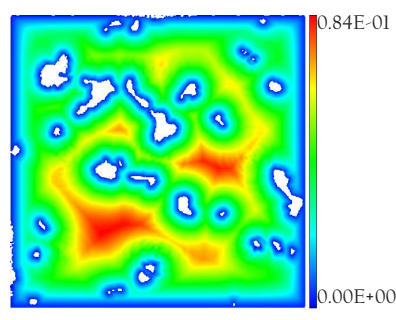

(d)

Figure 3.24: Geometry modeling of $\mathrm{TaC}$ (a) Grayscale image of the microstructure (b) Binary representation of the geometry (c) Sampled Euclidean distances (d) Distance field functions

of generating distance field functions from two dimensional images. Source of geometry for numerical experiments here are 2D micrographs taken from material's microstructure and transferred into distance field representation.

\section{Spark plasma sintered TaC}

In this numerical experiment a SEM image of a spark plasma sintered $\mathrm{TaC}$ is used as the source of geometry, the sample was prepared at $1850^{\circ} \mathrm{C}$ at a pressure of $100 \mathrm{MPa}$ and a hold time of 10 min. Fig. 3.24(a) shows the matrix and porosities, elastic modulus and Poisson's ratio obtained from nanoindentations for matrix are $E=$ $313 G P a, \nu=0.24$ [3]. Same as previous examples a rectangular RVE is considered, here the RVE contains arbitrarily shaped and distributed porosities. According to work flow diagram of the platform (Fig. 3.6) the SEM image is imported into geometric block as the native representation of microstructure. Then it is processed with a noise reduction filter and converted into a binary black and white image. Sampled Euclidean distances are generated based on the binary image by using Saito algorithm for Euclidean distance transformation. Eventually smooth (differentiable) distance filed functions are rendered by a least square fit of bilinear B-splines on a $285 \times 285$ grid on the samples of discrete Euclidean distances. Figs. 3.24(b)-(d) 


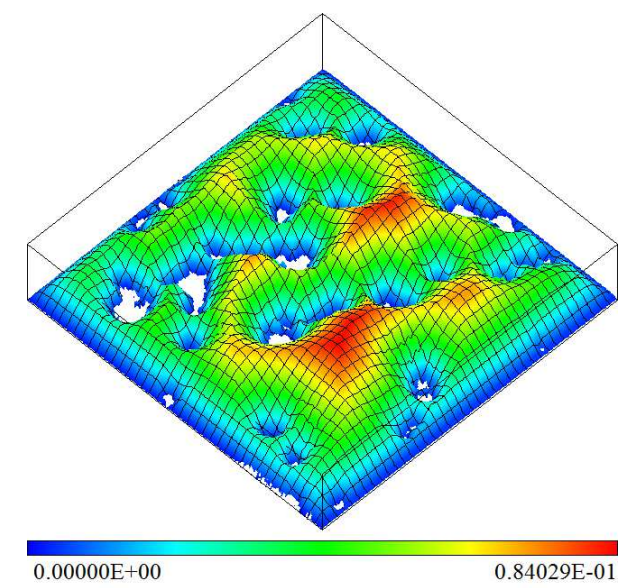

Figure 3.25: 3D presentation of distance field functions constructed over the SEM image of spark plasma sintered TaC.

illustrate the steps performed on the original image by the geometric block and also for having a more clear picture of the distance field functions created over the domain, their 3D plot is shown in Fig. 3.25.

Distance field functions are imported into MeshFree block and similar steps as previous samples are followed to derive the homogenized properties. Fig. 3.26(a) and (b) show the nonconforming grid constructed over the domain and Gauss integration point distribution. $\chi$ functions are presented in Fig. 3.27 and eventually homogenized properties are computed and compared with the outputs of strain energy method in Table 3.2.

In this sample, considering the image-based nature of the geometry representation, an open source platform $(\mathrm{OOF} 2)$ is used as the finite element solver. OOF (Object Oriented Finite element) is a group of FE solvers which studies the material's macroscopic behavior based on its microstructure, in this solver an image of microstructure is used as the source of geometry and a finite element mesh is generated by using an adaptive mesh technology which makes it possible to generate a geometry conforming mesh on different phases of structure. These phases are se- 


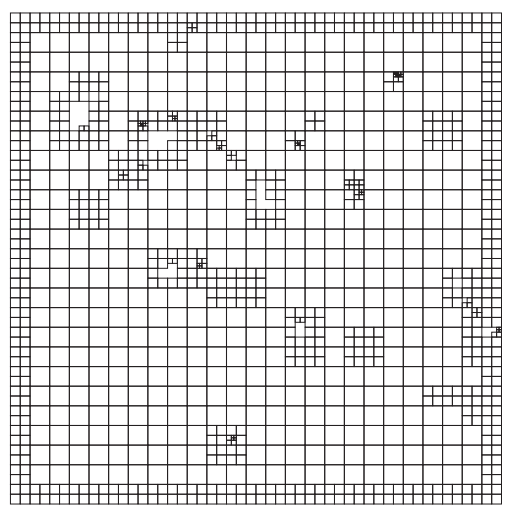

(a)

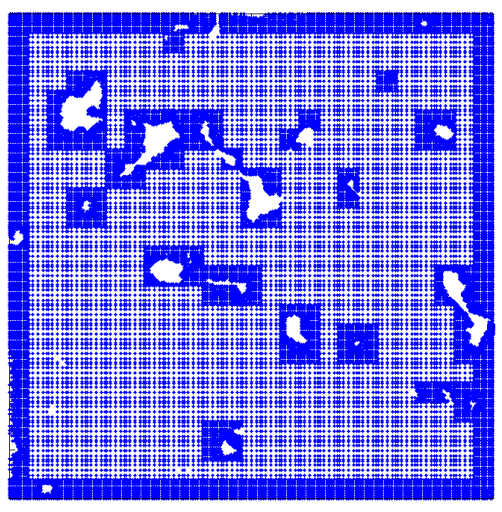

(b)

Figure 3.26: Geometry modeling of TaC (a) Nonconforming grid over the domain; (b) Gauss integration points distribution.
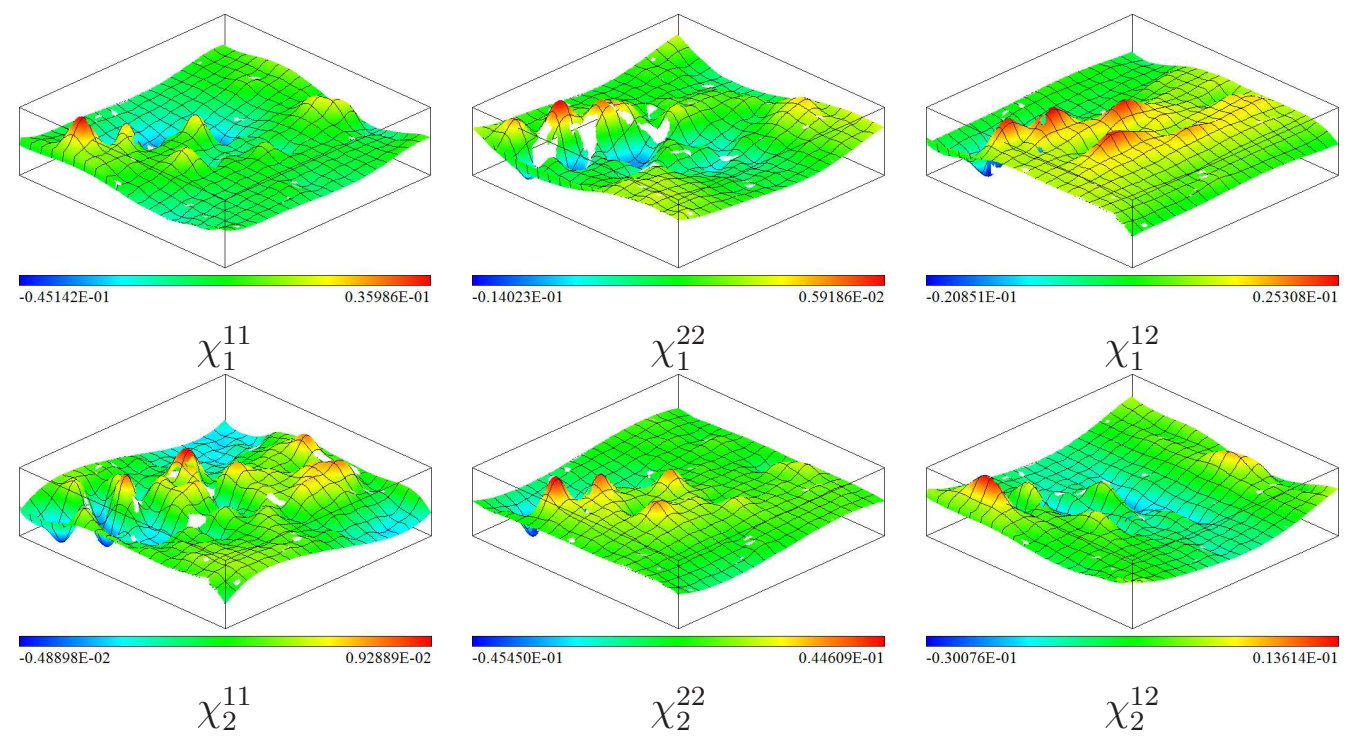

Figure 3.27: Basis $\left\{\chi_{m}^{k l}\right\}$ functions for the SEM image of spark plasma sintered TaC. 
Table 3.2: Homogenized stiffness matrix for the RVE obtained from SEM image of TaC. Values are derived by assuming plane stress and $E=313 G P a, \nu=0.24$.

\begin{tabular}{lccc}
\hline & Strain Energy Method & MeshFree / Asymptotic & Difference \\
\hline$E_{1111}^{H}$ & 291.87 & 304.02 & $4.16 \%$ \\
$E_{2222}^{H}$ & 292.60 & 304.96 & $4.22 \%$ \\
$E_{1212}^{H}$ & 113.65 & 117.48 & $3.37 \%$ \\
$E_{2211}^{H}$ & 70.62 & 73.74 & $4.42 \%$ \\
$E_{1211}^{H}$ & -0.45 & -0.36 & $20.00 \%$ \\
$E_{1222}^{H}$ & -0.34 & -0.18 & $47.06 \%$ \\
\hline
\end{tabular}

lected according to their colors and different mechanical properties can be assigned to each one of them $[38,9]$.

Fig. 3.28 shows a sample setup of OOF2 software with an image imported in it as the source of domain geometry. Three sets of KUBC boundary conditions compatible with Hill principle are applied to calculate homogenized properties of the domain by strain energy method:

$$
\begin{array}{cl}
\text { Set I: }\left.u_{x}\right|_{\partial Y}=x & \left.u_{y}\right|_{\partial Y}=0 \\
\text { Set II: }\left.u_{x}\right|_{\partial Y}=0 & \left.u_{y}\right|_{\partial Y}=y \\
\text { Set III: }\left.u_{x}\right|_{\partial Y}=0.5 y & \left.u_{y}\right|_{\partial Y}=0.5 x
\end{array}
$$

Even for the geometry used here, which is a relatively simple one, the initial mesh generated by OOF2 which is basically a grid over the domain (Fig. 3.28) has to go through considerable amount of manual modifications to minimize its effective energy. Effective energy of a mesh structure depends on the material and shape homogeneity of its elements, for a mesh containing elements with completely homogenous material and shape this value goes toward zero and as the inhomogeneity increases the effective energy approaches to one. Fig. 3.29 shows the modifications performed here which involves subdividing elements with heterogeneity in material, 


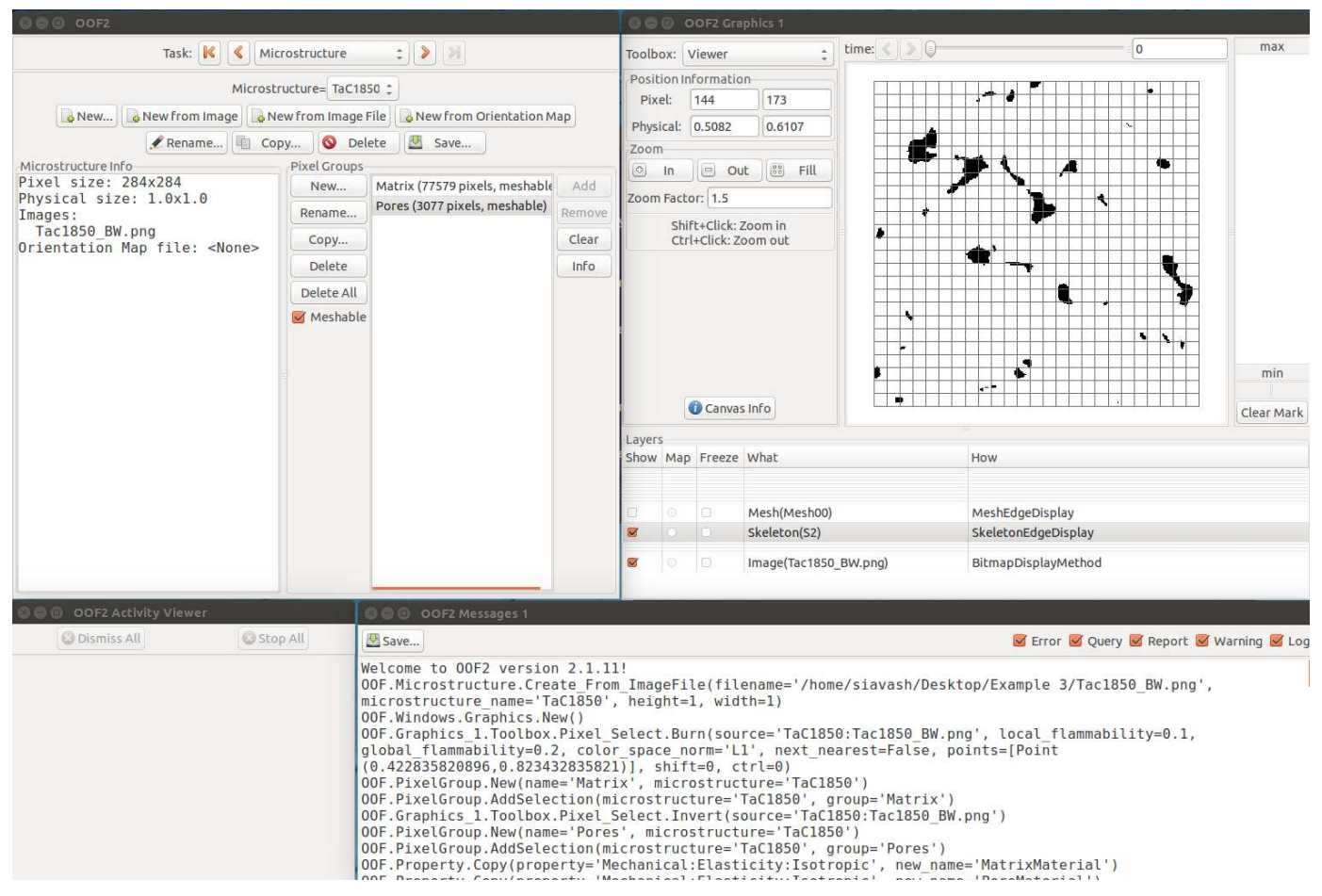

Figure 3.28: OOF2 software setup with a microstructure image imported as source of the geometry.

snapping the internal boundary nodes to boundary limits and element annealing [38].

In Fig. 3.29(d) some areas are marked and numbered on opposing edges, which indicates the regions which mesh still needs modifications to be able to be used in a node pairing scheme for a boundary value problem with periodic boundary conditions.

\section{AlSi-dispersed CNT matrix}

In continuation of testing performance of the developed hybrid platform, another SEM image is used as the source of geometry and the workflow shown in Fig. 3.6 is followed to calculate its homogenized properties. Figs. 3.30(a)-(c) respectively show the original SEM image, the binary representation of the geometry and the 


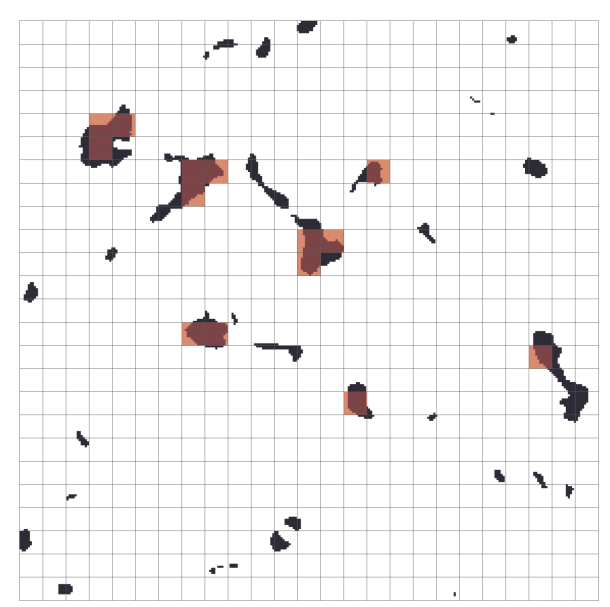

(a)

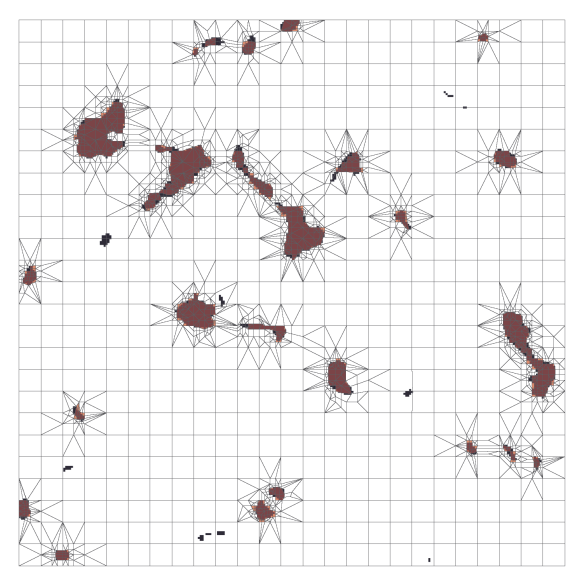

(c)

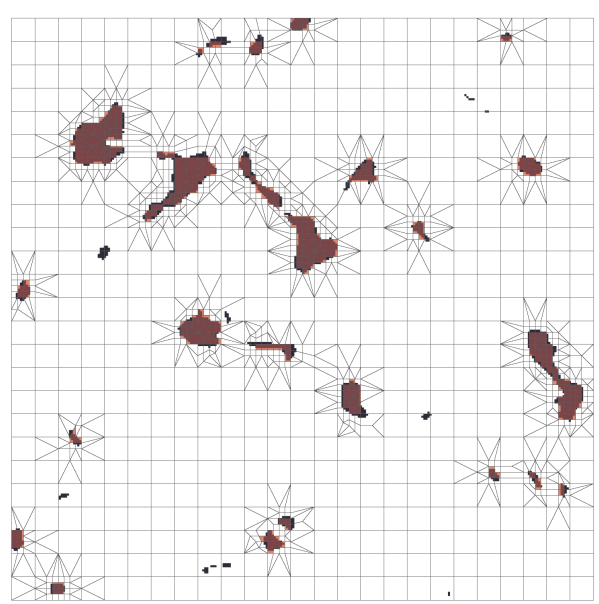

(b)

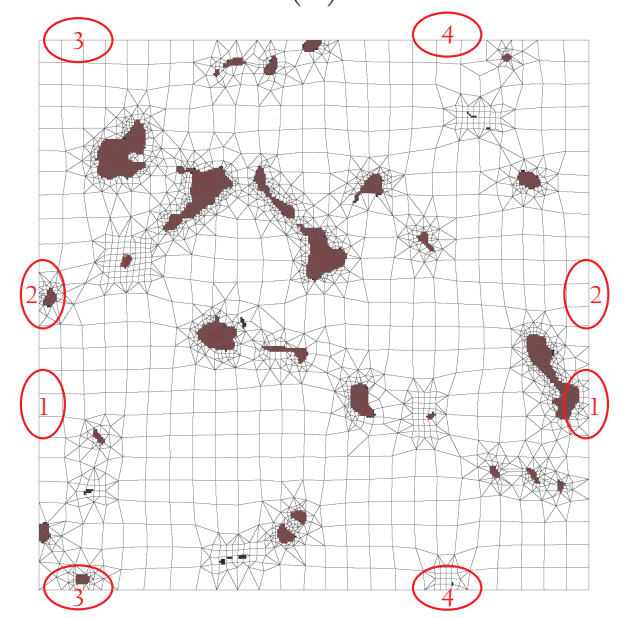

(d)

Figure 3.29: (a) The initial mesh on the TaC microstructure image (b) After refining the mesh in vicinity of porosities (c) After snapping the nodes close to internal boundaries to the boundaries and smoothing the elements (d) After performing steps $b$ and $c$ for elements which are still consisted of two different materials and rationalizing and fixing illegal elements; Elements representing the porous area are colored to show the captured geometry by OOF2. Marked areas with same numbers show the regions which mesh still needs modifications to have matching node distribution on opposing edges in order to perform node pairing to satisfy periodic boundary conditions. 


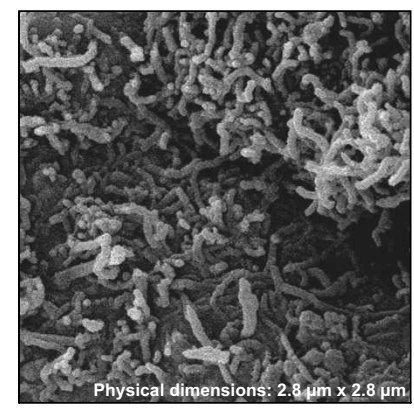

(a)

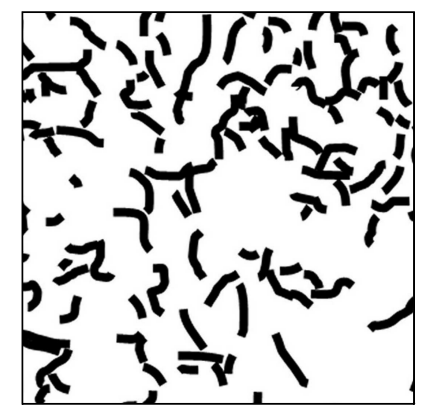

(b)

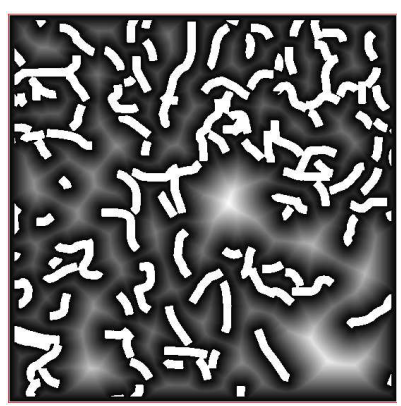

(c)

Figure 3.30: Geometry modeling of AlSi-CNT (a) SEM image of the microstructure (b) Binary representation of the geometry (c) Sampled Euclidean distances.

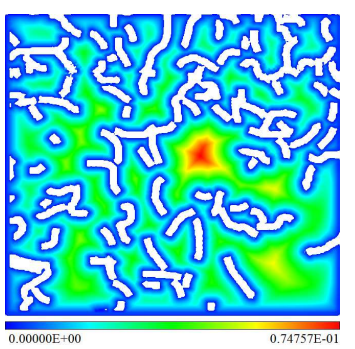

(a)

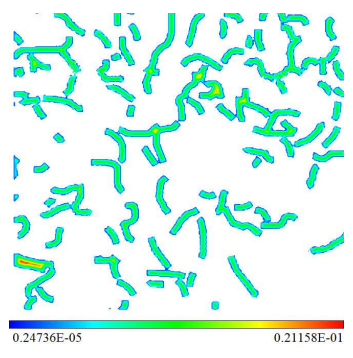

(b)

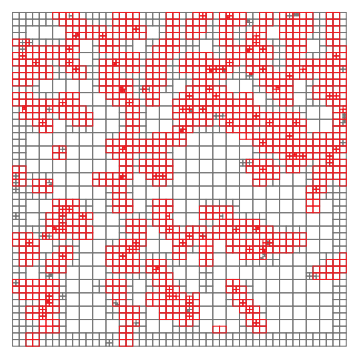

(c)

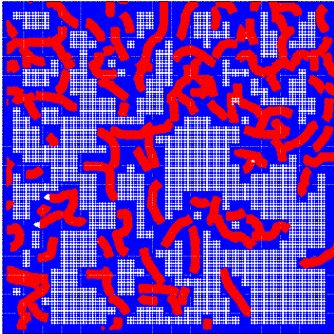

(d)

Figure 3.31: Geometry modeling of AlSi-CNT (a) Distance field representation of the matrix (b) Distance field representation of the fibers (c) Nonconforming grid for both phases (d) Gauss integration points distribution.

corresponding Euclidean distances. Over a $540 \times 540$ grid of bilinear B-splines, differentiable distance field functions are generated from Euclidean distances for both phases in the microstructure. Then nonconforming grid is created over the domain and Gauss integration points are distributed as shown in Fig. 3.31.

The above information is imported into meshfree solver and by assuming a plane stress state and following material properties: $E_{\text {matrix }}=74.52 G P a, \nu_{\text {matrix }}=0.33$, $E_{\text {fiber }}=600 \mathrm{GPa}$ and $\nu_{\text {fiber }}=0.27$ values of $\chi$ functions and eventually homogenized mechanical properties of the AlSi-CNT are calculated. Fig. 3.32 shows the $\chi$ functions and homogenized properties are presented in Table 3.3. The geometry 

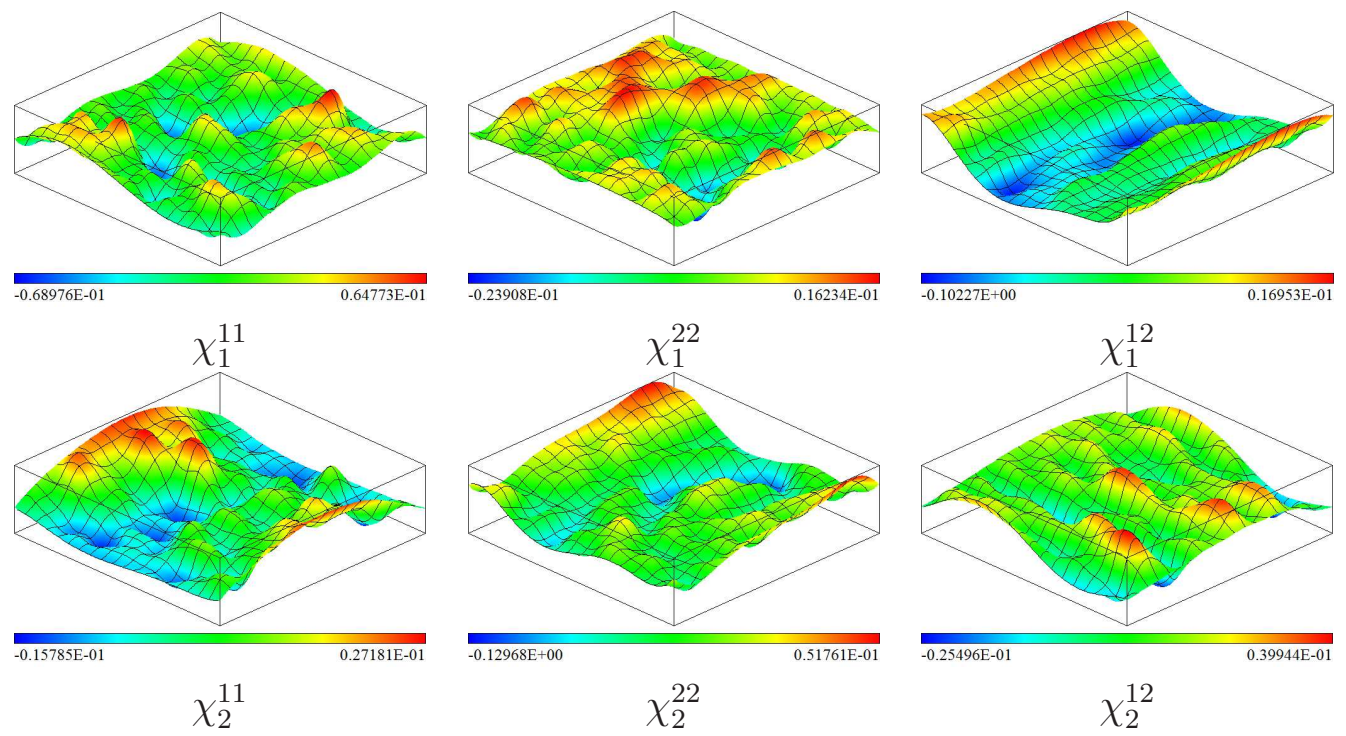

Figure 3.32: Basis $\left\{\chi_{m}^{k l}\right\}$ functions for the SEM image of AlSi-CNT.

Table 3.3: Homogenized stiffness matrix for the RVE representing AlSi-CNT. Values are derived by assuming plane stress and $E_{\text {matrix }}=74.52 G P a, \nu_{\text {matrix }}=0.33$, $E_{\text {fiber }}=600 G P a$ and $\nu_{\text {fiber }}=0.27$.

\begin{tabular}{lccc}
\hline & Strain Energy Method & MeshFree / Asymptotic & Difference \\
\hline$E_{1111}^{H}$ & 122.12 & 130.42 & $6.80 \%$ \\
$E_{2222}^{H}$ & 121.08 & 129.82 & $7.22 \%$ \\
$E_{1212}^{H}$ & 39.98 & 46.88 & $17.26 \%$ \\
$E_{2211}^{H}$ & 36.78 & 42.98 & $16.86 \%$ \\
$E_{1211}^{H}$ & -0.50 & -0.53 & $6.00 \%$ \\
$E_{1222}^{H}$ & -0.18 & -0.23 & $27.78 \%$ \\
\hline
\end{tabular}




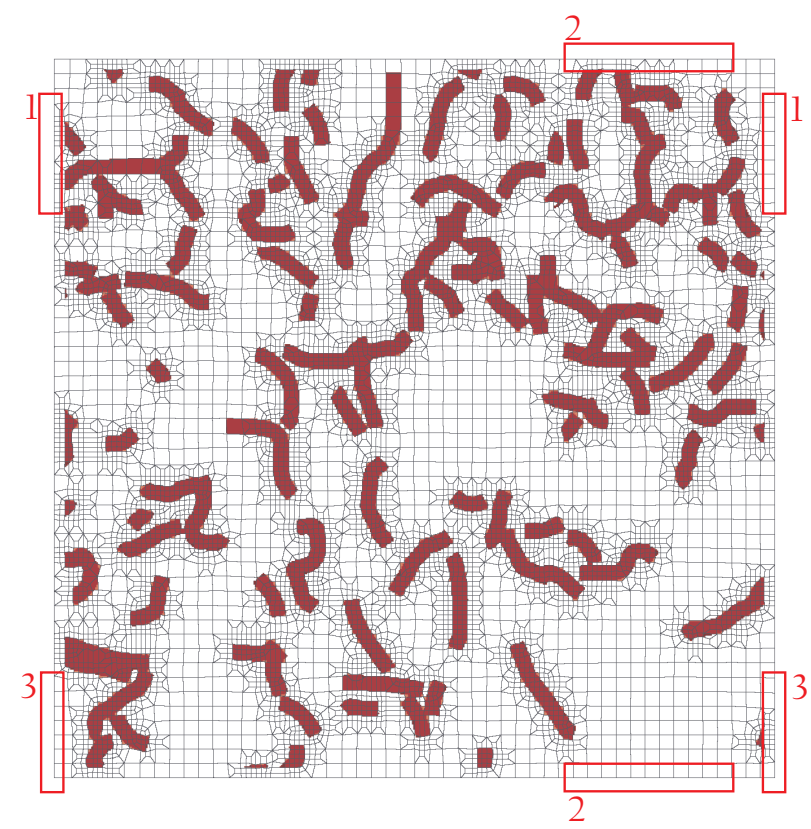

Figure 3.33: The final finite element mesh for AlSi-CNT, created with OOF2 and manually modified. Areas which still needs modifications for performing node pairing are marked.

presented in Fig. 3.30(b) is also imported in to OOF2 to calculate the homogenized properties of the structure with strain energy method. A finite element mesh is generated on the domain and the mesh is gone through considerable amount of manual modifications to be compatible with geometric features of the domain. Final mesh is shown in Fig. 3.33 and same as the mesh created in Fig. 3.29, the areas which still requires modifications to make the mesh suitable for a boundary value problem with periodic boundary conditions are marked.

\section{Al-10-CNT}

The last numerical experiment which takes an image as source of the geometry investigates the homogenized properties of another composite with carbon nanotubes shown in Fig. 3.34(a). Same as before this image is processed an converted to a black and white binary image, then corresponding Euclidean distances are generated 


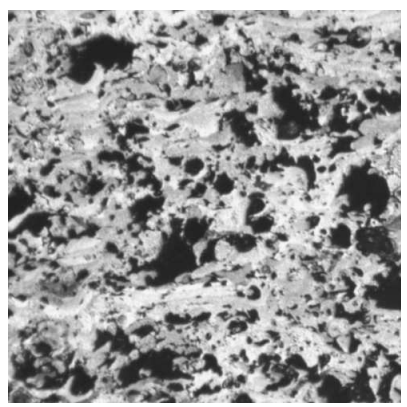

(a)

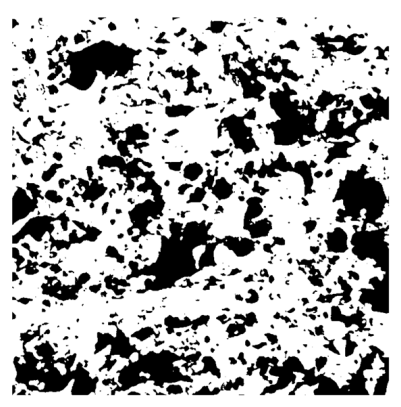

(b)

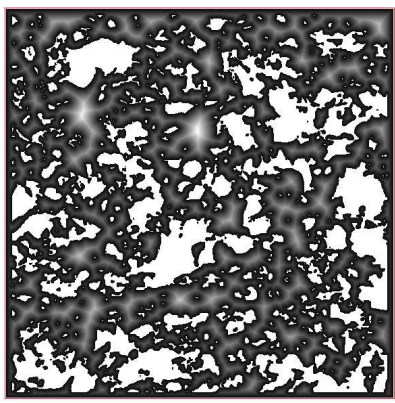

(c)

Figure 3.34: Geometry modeling of Al-10-CNT (a) Grayscale image of the microstructure (b) Binary representation of the geometry (c) Sampled Euclidean distances.

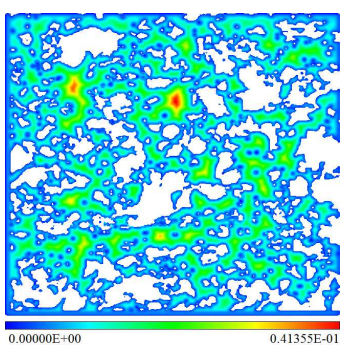

(a)

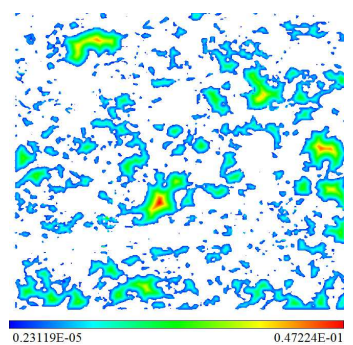

(b)

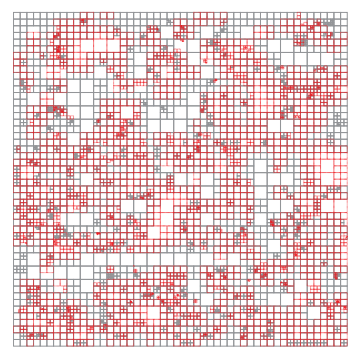

(c)

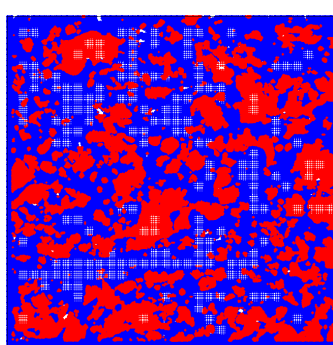

(d)

Figure 3.35: Geometry modeling of Al-10-CNT (a) Distance field representation of the matrix (b) Distance field representation of the fibers (c) Nonconforming grid for both phases (d) Gauss integration points distribution.

as shown in Fig. 3.34(c). Figs. 3.35(a)-(d) respectively show the distance field functions for matrix and fibers which are created over a $570 \times 570$ grid of bilinear Bsplines, the nonconforming grid and distribution of Gauss integration points. Same material properties as $A l S i-C N T$ are assumed for calculation of $\chi$ functions and behavior of these functions are presented in Fig. 3.36. Consequently homogenized properties are computed and compared to outcomes of strain energy method in Table 3.4. Fig. 3.37 shows the manually modified mesh which was originally created over the domain by OOF2 and the problematic areas for node pairing are marked. 

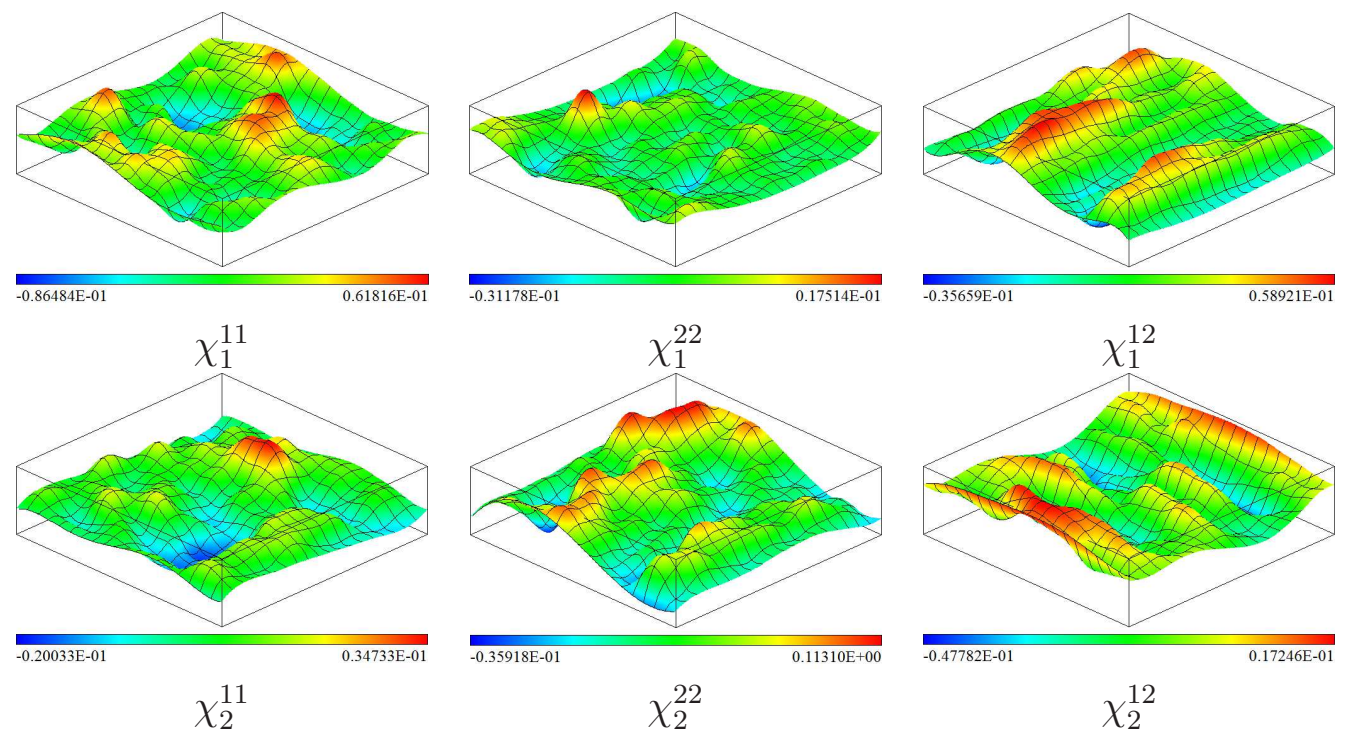

Figure 3.36: Basis $\left\{\chi_{m}^{k l}\right\}$ functions for the SEM image of Al-10-CNT.

Table 3.4: Homogenized stiffness matrix for the RVE representing Al-10-CNT. Values are derived by assuming plane stress and $E_{\text {matrix }}=74.52 G P a, \nu_{\text {matrix }}=0.33$, $E_{\text {fiber }}=600 G P a$ and $\nu_{\text {fiber }}=0.27$.

\begin{tabular}{lccc}
\hline & Strain Energy Method & MeshFree / Asymptotic & Differences \\
\hline$E_{1111}^{H}$ & 171.45 & 189.94 & $10.26 \%$ \\
$E_{2222}^{H}$ & 158.09 & 178.37 & $12.83 \%$ \\
$E_{1212}^{H}$ & 50.80 & 56.04 & $10.31 \%$ \\
$E_{2211}^{H}$ & 45.80 & 50.62 & $10.52 \%$ \\
$E_{1211}^{H}$ & 0.23 & 0.18 & $21.74 \%$ \\
$E_{1222}^{H}$ & -0.34 & -0.26 & $23.53 \%$ \\
\hline
\end{tabular}




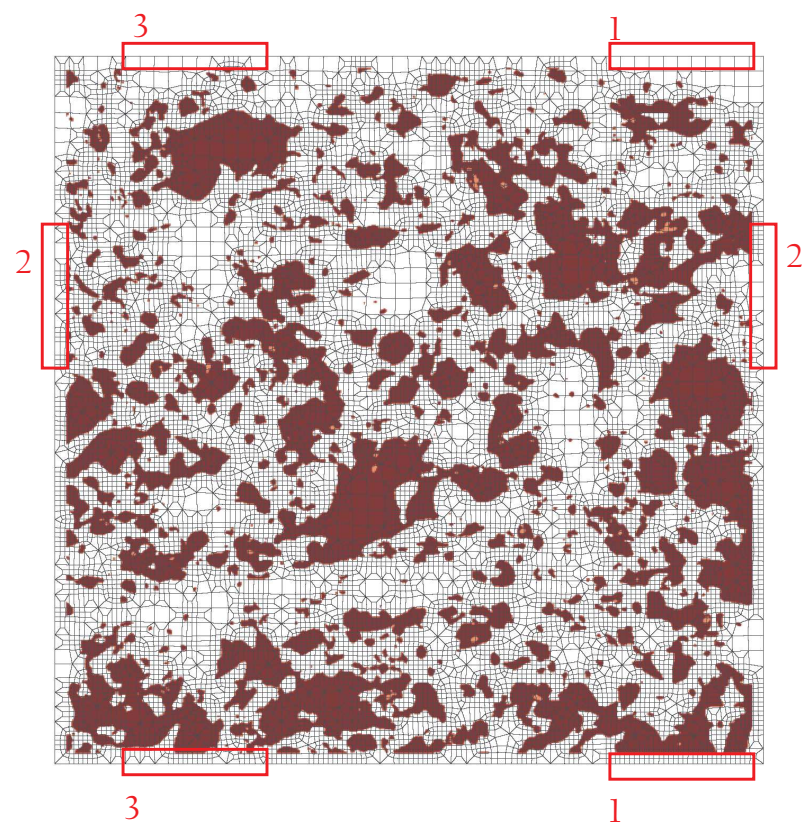

Figure 3.37: The final finite element mesh created over Al-10-CNT domain by OOF2 and manually modified. Areas which still needs modifications for performing node pairing are marked.

\section{Discussion}

Going back to Tables 3.2-3.4 and reviewing the differences shows that in some cases, where geometries are captured from images, there are considerable differences between results of Meshfree/Asymptotic and Strain Energy methods. It should be noted there are very few software packages capable of converting images into finite element models and a very fewer number available as open source platforms. Although among these platforms OOF2 suits homogenization problem the best, it has theoretical differences with Meshfree/Asymptotic platform which can explain the differences reported in homogenized properties.

Meshfree/Asymptotic platform uses approximate distance field functions to capture a realistic representation of the geometry from micrograph images where in OOF2 a finite element model is manually created using a series of mesh modification tools. 
In OOF2 homogenized properties of the domain are calculated by applying Hill's conditions (see Chapter 2.1.3) and integrating strain energy, but Meshfree/Asymptotic platform uses Asymptotic homogenization technique and finally for integration, MeshFree/Asymptotic platform uses Gauss integration points with adjustable resolution where in OOF2 all integration points are given equal weights.

As stated in Section 3.1.1 one of the main advantages of meshfree/asymptotic platform is the elimination of meshing step from solution procedure and satisfying periodic boundary conditions via enrichment functions. Going back to Figs. 3.29, 3.33 and 3.37 clearly shows that how meshing process for geometries acquired from images can be time consuming and demanding and meshfree solution structure can help us to perform the homogenization in complete autonomy. In addition to easy handling of boundary conditions, comparing above figures with their corresponding distance field representations which respectively are Figs. 3.24, 3.31 and 3.35 shows that the developed platform can also capture a more accurate model of microstructure in comparison to conventional finite elements. 


\section{CHAPTER 4 \\ STRESS AND STRAIN DISTRIBUTION}

As mentioned earlier in Section 2.1.1 one of the main advantages of asymptotic method to other homogenization techniques is that with this method in addition to finding homogenized properties of the material, the full structural problem at micro level can also be solved. This method explicitly defines equations to calculate the micro-scale stress and strain levels and provides the means to study the effect of micro-level geometric features on stress distribution.

In this section we will start by going over the mathematical formulation of stress and strain fields and explain how they can be calculated without fully modeling all the geometric features of the domain, then performance of meshfree/asymptotic method in solving the micro level structural problem will be tested by comparing the results to the data obtained from studying full geometric models.

\subsection{Stress-Strain Localization Methodology}

Going back to Chapter 2, we recall that in asymptotic method behavior of any physical field which can be related to two distinct macro-scale and micro-scale domains can be described as an expansion in form of eq. (2.2). Therefore displacement, being one of the physical fields depending on both macro and micro scale, can be written as:

$$
\boldsymbol{u}^{\epsilon}(\boldsymbol{x})=\boldsymbol{u}^{0}(\boldsymbol{x})+\epsilon \boldsymbol{u}^{1}(\boldsymbol{x}, \boldsymbol{y})
$$

It was also explained that in asymptotic expansion of displacement, the first term on the right hand side of the equation is in fact displacement fields calculated via homogenized properties and second term could be computed by eq. (2.16):

$$
u_{i}^{1}=-\chi_{i}^{k l}(\boldsymbol{x}, \boldsymbol{y}) \frac{\partial u_{k}^{0}(\boldsymbol{x})}{\partial x_{l}}+\tilde{u}_{i}^{1}(\boldsymbol{x})
$$


After computing $\boldsymbol{u}^{0}(\boldsymbol{x})$ and $\boldsymbol{u}^{1}(\boldsymbol{x}, \boldsymbol{y})$, eqs. (2.28) and (2.29) could be used to find out stress and strain fields. Here to make it easier to follow the described procedure, mentioned equations are recalled from Chapter 2 and rewritten:

$$
\begin{gathered}
\varepsilon_{i j}^{\epsilon}=\varepsilon_{i j}^{0}+\epsilon^{1}(\ldots) \approx \frac{1}{2}\left[\left(\frac{\partial u_{i}^{0}}{\partial x_{j}}+\frac{\partial u_{j}^{0}}{\partial x_{i}}\right)+\left(\frac{\partial u_{i}^{1}}{\partial y_{j}}+\frac{\partial u_{j}^{1}}{\partial y_{i}}\right)\right] \\
\sigma_{i j}^{\epsilon}=\sigma_{i j}^{0}+\epsilon^{1}(\ldots) \approx \frac{1}{2} E_{i j k l}^{\epsilon}\left[\left(\frac{\partial u_{k}^{0}}{\partial x_{l}}+\frac{\partial u_{l}^{0}}{\partial x_{k}}\right)+\left(\frac{\partial u_{k}^{1}}{\partial y_{l}}+\frac{\partial u_{l}^{1}}{\partial y_{k}}\right)\right]
\end{gathered}
$$

The process for stress-strain field localization by using meshfree/asymptotic platform could be summarized as:

- Computing $\chi$ functions and finding homogenized elasticity tensor $E^{H}$.

- Using the homogenized elasticity tensor to calculate $\boldsymbol{u}^{0}(\boldsymbol{x})$.

- Calculate $\boldsymbol{u}^{1}(\boldsymbol{x}, \boldsymbol{y})$ values by substituting $\boldsymbol{u}^{0}(\boldsymbol{x})$ and $\boldsymbol{\chi}$ values in eq. (2.16)

- Plugging $\boldsymbol{u}^{0}(\boldsymbol{x})$ and $\boldsymbol{u}^{1}(\boldsymbol{x}, \boldsymbol{y})$ values in eqs. (4.2) and (4.3) to calculate localized stress and strain fields.

\subsection{Numerical Experiments}

Numerical experiments presented here, are generated by using the RVEs introduced in Chapter 3. Different numbers of previously studied RVE geometries are put together here to construct a global domain with a periodic structure. Each domain is subjected to a combination of different type of boundary conditions (axial or shear forces or displacements) to simulate different real life situations. Then in conjunction with these boundary conditions, the existing data on the RVEs homogenized

properties $\left(E^{H}\right)$ are used to solve the simple homogenized elasticity problem for 
homogenized displacements, $\boldsymbol{u}^{0}(\boldsymbol{x})$. Previously computed $\boldsymbol{\chi}$ functions and homogenized displacements are plugged in eq. (2.16) to calculate $\boldsymbol{u}^{1}(\boldsymbol{x}, \boldsymbol{y})$ functions, which here we call them Geometric Effects. Eventually by having all the necessary information to find out the total displacement values, they are used to localize stress and strain distributions and study the effect of different geometric features on mentioned functions.

To evaluate the performance of meshfree/asymptotic platform, the global domain geometry for each numerical experiment has been also fully modeled in a meshfree solver for elasticity problems and displacement, strain and stress distributions are calculated. In each case results are compared with each other and corresponding error distributions are presented.

\subsubsection{Carbon/Epoxy composite}

In this example the RVE presented in Fig. 3.8 is used to construct global periodic domains. Carbon/Epoxy RVEs are put together in three different geometric setups which are made from $2 \times 2,10 \times 10$ and $50 \times 50$ rectangular grids of RVEs. Material properties are the same as Chapter 3 and the domains undergo a plane strain deformation. Fig. 4.1(a) shows the geometry and properties of the RVE and global domains assembled from 4 and 100 RVEs are presented in Figs. 4.1(b) and (c), as shown the left edge of domain is fixed in $x$ and $y$ directions and the constant shear force of $100 \mathrm{~N} /$ unit length is applied to the right edge. According to stressstrain localization process, in the first step $\chi$ functions and homogenized properties are calculated, which for the mentioned RVE it has been already done in Chapter 3. Then elasticity problem for the homogenized domain is solved with the applied boundary conditions. Fig. 4.1(d) shows the setup for homogenized problem, and it 


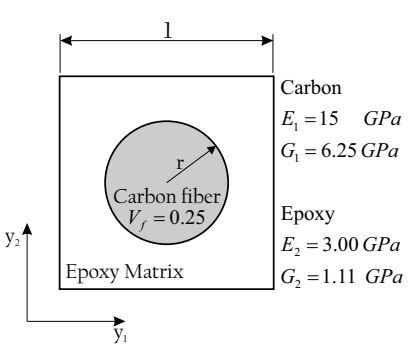

(a)

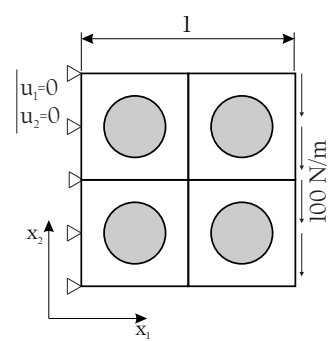

(b)

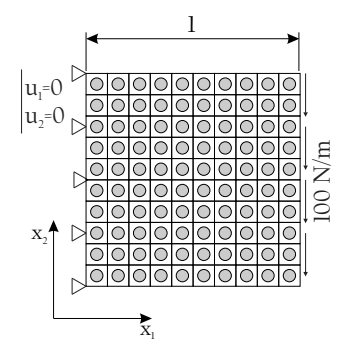

(c)

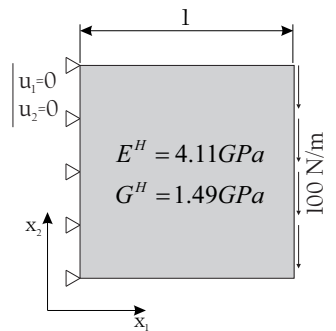

(d)

Figure 4.1: Geometry modeling (a) RVE; (b) Global domain with a $2 \times 2$ grid and boundary conditions ; (c) Global domain with a $10 \times 10$ grid and boundary conditions(d) Homogenized problem.

is solved for $\boldsymbol{u}^{0}(\boldsymbol{x})$. Now that $\boldsymbol{u}^{0}(\boldsymbol{x})$ functions are computed and values of $\chi$ are known, geometry effect functions can be calculated by using eq. (2.16).

Going back to eq. (2.1) and by comparing the unit lengths in local and global coordinates in Fig. 4.1 we can see that the values of $\epsilon$ for the global domains with a $2 \times 2,10 \times 10$ and $50 \times 50$ grid are respectively equal to $0.5,0.1$ and 0.02 . Substituting $\epsilon$ and values for $\boldsymbol{u}^{0}$ and $\boldsymbol{u}^{1}$ in eq. (4.1), we can calculate total displacements for each global domain setup. After calculating $\boldsymbol{u}^{0}$ and $\boldsymbol{u}^{1}$ functions, strain and stress distributions can be computed by plugging in displacement functions in eqs. (4.2) and (4.3).

At the same time to evaluate the results of meshfree/asymptotic platform, the geometry for all three global domains are fully modeled with a meshfree geometric engine. This model contains all the geometric features and their corresponding material properties and the full structural problem is solved for it by considering the same boundary conditions as the homogenized problem and choosing a proper solution structure from Table 2.1.

Using the results of meshfree solution on full geometric model (FGM) as a bench mark and comparing outputs of meshfree/asymptotic platform with them, we can calculate $L_{2}$-norms for each member of displacement vector and strain and stress 


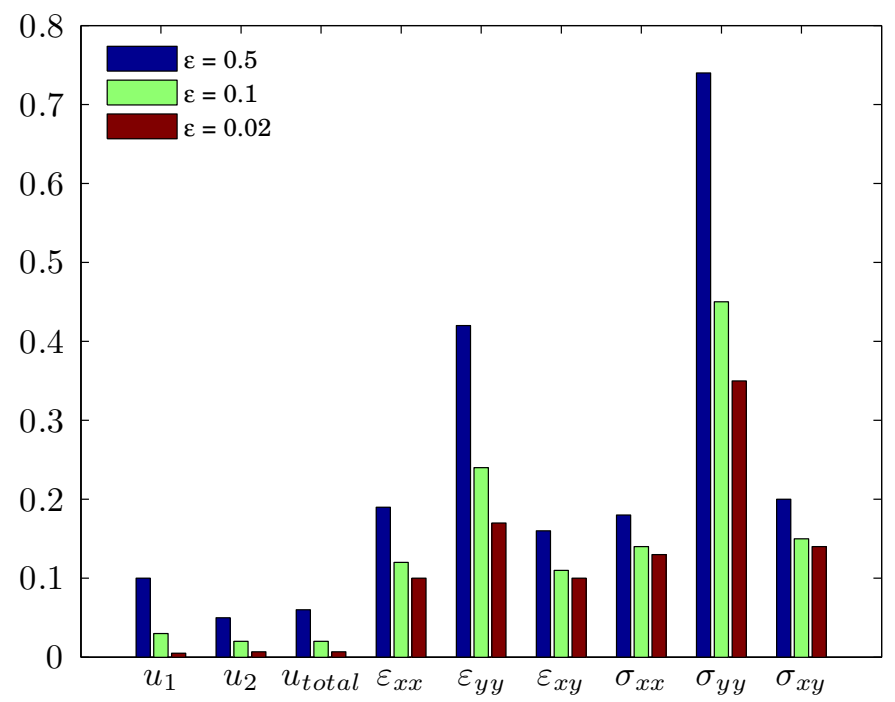

Figure 4.2: $L_{2}$-norms calculated for strains and stresses with different values of $\epsilon$. tensors by:

$$
L_{2}-\text { norm }=\sqrt{\frac{\int_{\Omega}\left(f^{r e f}-f^{\epsilon}\right)^{2} d \Omega}{\int_{\Omega} f^{r e f^{2}} d \Omega}}
$$

Recalling eq. (2.2), we know that values calculated by asymptotic expansion should get closer to results of full geometric solution as $\epsilon \rightarrow 0^{+}$. In Fig. $4.2 \mathrm{~L}_{2^{-}}$ norms for displacements, strains and stresses of global domains with $\epsilon=0.5,0.1,0.02$ are computed and presented side by side each other and it clearly can be observed that as the value of $\epsilon$ gets smaller results of homogenized solution gets closer to outcomes of full geometric model.

$L_{2}$-norm gives us a quantitative understanding about the performance of meshfree/asymptotic platform, but in order to see how errors are distributed over the domain and in which areas it is more likely to have larger error values, error distributions for displacements, strains and stresses are also calculated by using

$$
f^{\text {error }}(\boldsymbol{x})=\frac{\left|f^{r e f}(\boldsymbol{x})-f^{\epsilon}(\boldsymbol{x})\right|}{\max \left(\left|f^{r e f}(\boldsymbol{x})\right|\right)}
$$

Figs. 4.3, 4.4 and 4.5 respectively show the error distributions for displacements, strains and stresses. In each figure the first row corresponds to a global domain with 
$\epsilon=0.5$, second row presents the error distribution when $\epsilon=0.1$ and the last row is computed with $\epsilon=0.02$. Studying error distributions shows that in predicting displacement values, asymptotic/meshfree platform performs very well even with large size RVEs and as $\epsilon$ gets smaller the answers of full geometric model and homogenized model become almost the same (largest error value is less than 1\%).

For strains and stresses although maximum error values are larger comparing to displacements, but if we look closer we can see that large values of error is mostly limited to boundary RVEs. And as $\epsilon$ gets smaller size of RVEs and consequently the area occupied by boundary RVEs, which is the area containing large values of error, gets smaller and at all the other points of domain results of full geometric model and homogenized model are in very good agreement with each other.

Going back to Chapter 3 and reviewing eq. (3.6) shows that solution structure proposed for homogenization problem is designed in a way to satisfy periodic boundary conditions and when a RVE edge and one of the global domain edges coincide with each other, enforcing periodic boundary conditions on the boundary RVE will cause discrepancies between the two sets of results.

Studying $L_{2}$-norms and error distribution plots for different values of $\epsilon$ confirms that the results obtained from homogenized model with meshfree/asymptotic platform converge toward the outcomes of full geometric model. Here to give a more clear picture of the stress-strain localization process results of meshfree/asymptotic platform for the global domain with $\epsilon=0.1$ are also presented.

As explained earlier the first step after solving homogenized setup of the problem is to compute geometric effects and then use them to calculate displacement and eventually strain and stress values. Fig. 4.6 shows how inhomogeneities in the geometry can effect the homogenized displacement fields and Fig. 4.7 compares the total displacements calculated by asymptotic/meshfree platform with our bench- 
mark which is the results of full geometric model. Same comparison is performed for strains and stresses in Figs. 4.8 and 4.9. It is worth pointing out that although in calculation of strain and stress fields there is a slight error in the values, but the proposed platform is very well capable of predicting the location where maximum strains and stresses happen.

\subsubsection{Square RVE with a square void}

Same as the previous example, one of the RVEs studied in Chapter 3 is used here to construct a global domain. As shown in Fig. 4.10(a) the square RVE with a square void is considered as the local domain and it is assembled in three different $1 \times 4,5 \times 20$ and $25 \times 100$ grids to form global domains with different $\epsilon$ values. Figs 4.10(b) and 4.10(c) show the structure of the global domains with $1 \times 4$ and $5 \times 20$ grids and also the boundary conditions. The global domains have fixed supports on both ends and a distributed load with a constant profile is being applied on them in $-y$ direction. Material properties are the same as Chapter 3 and the domains experience a plane stress loading.

Following the same steps as Section 4.2.1, first homogenized setup of the problem which is shown in Fig. $4.10(\mathrm{~d})$ is solved to find homogenized displacements. Then $\boldsymbol{u}^{0}(\boldsymbol{x})$ values alongside with $\chi$ functions are used to find geometry effects and eventually total displacements are calculated. This process is performed for all three global domains and the corresponding $L_{2}$-norms for their displacements, strains and stresses are calculated with respect to the results of full geometric model analysis and presented in Fig. 4.11.

In addition to $L_{2}$-norms, error distributions are also calculated by equation 4.5 and the plots for displacement, strain and stress error distributions are respectively 

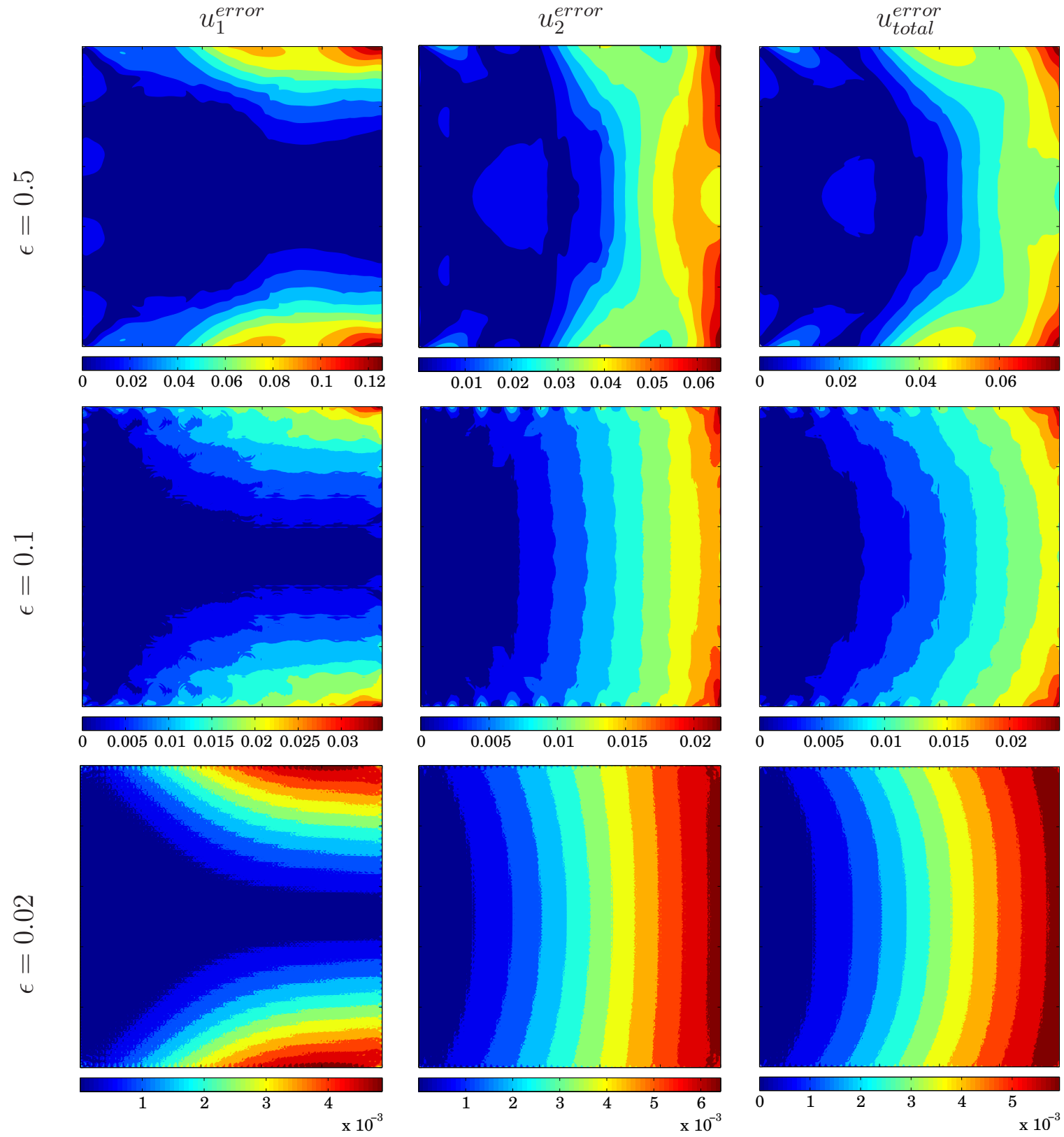

Figure 4.3: Error distribution for displacement over the global domains with $\epsilon=$ $0.5,0.1,0.02$. 

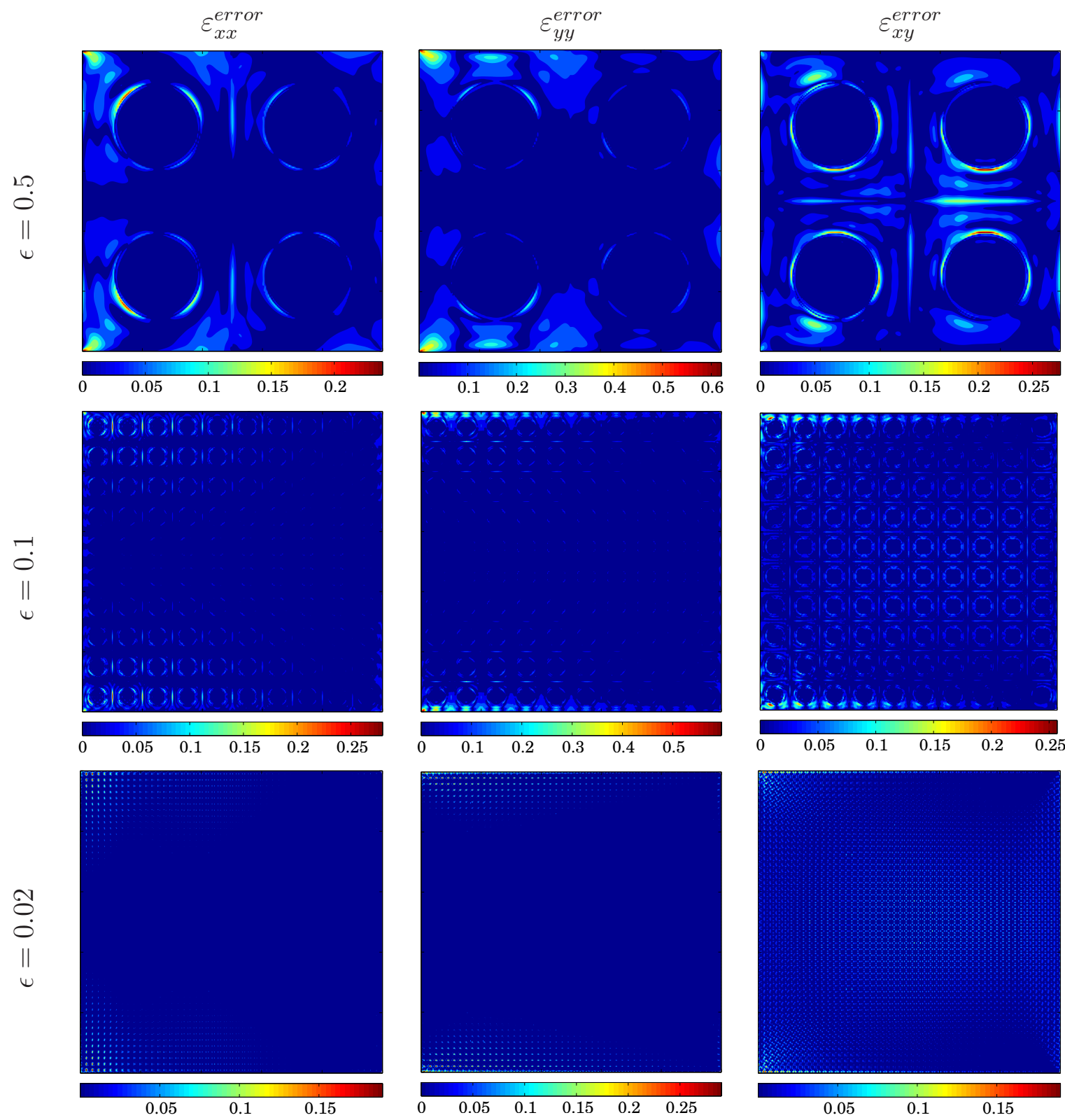

Figure 4.4: Error distribution for strains over the global domains with $\epsilon=$ $0.5,0.1,0.02$. 

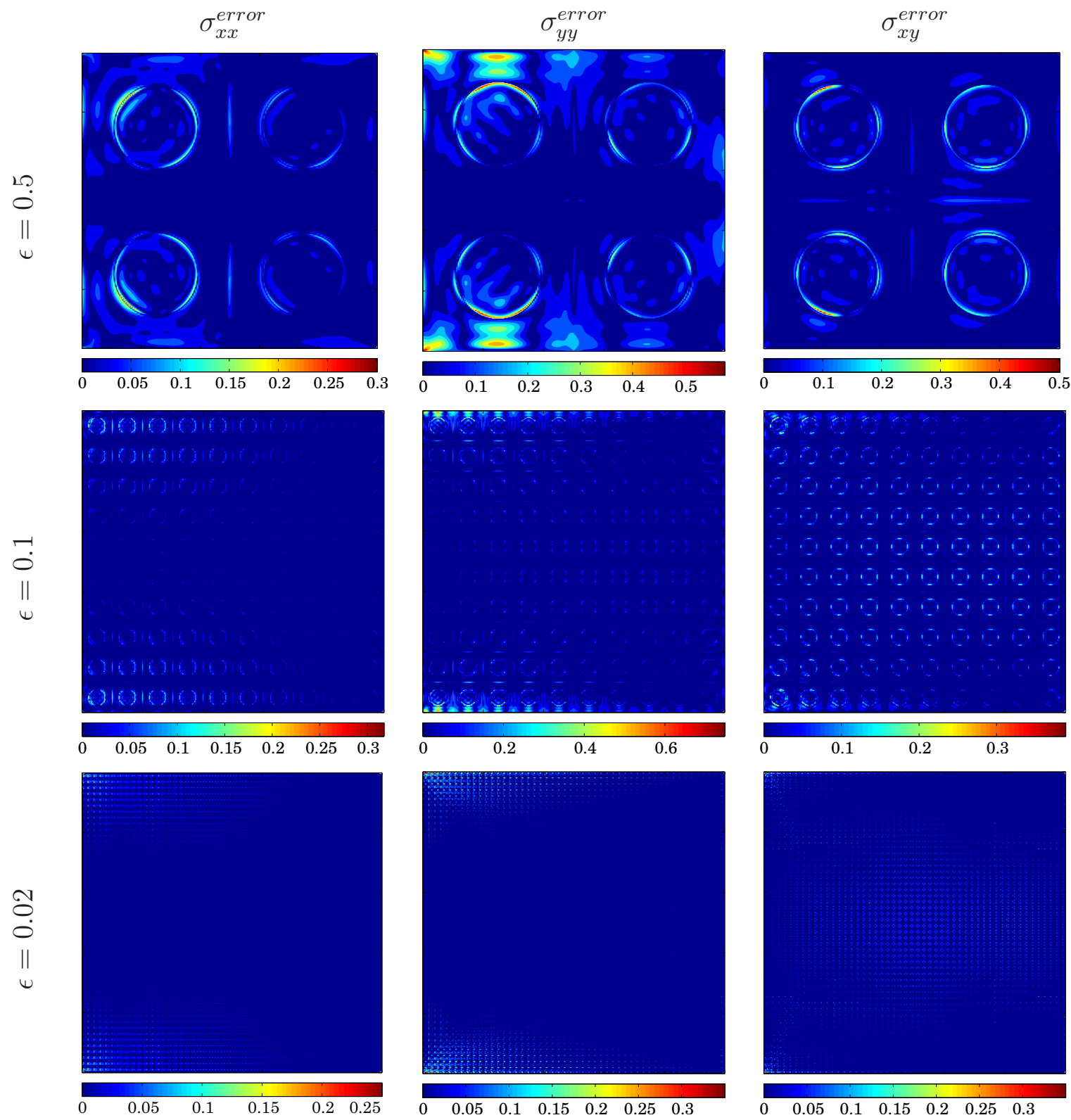

Figure 4.5: Error distribution for strains over the global domains with $\epsilon=$ $0.5,0.1,0.02$. 


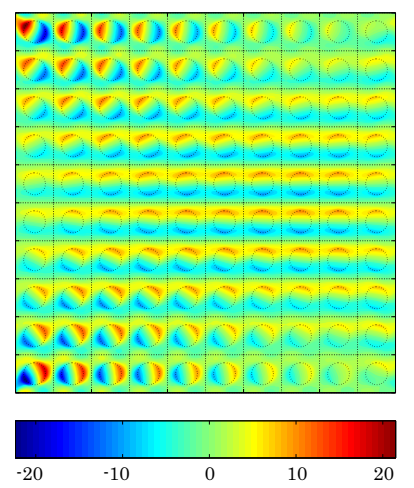

(a)

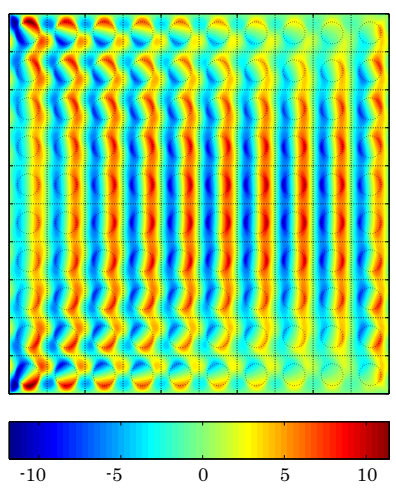

(b)

Figure 4.6: Geometry effects for the global domain with $\epsilon=0.1$ (a) $u_{1}^{1}(\boldsymbol{x}, \boldsymbol{y})$; (b) $u_{2}^{1}(\boldsymbol{x}, \boldsymbol{y})$.
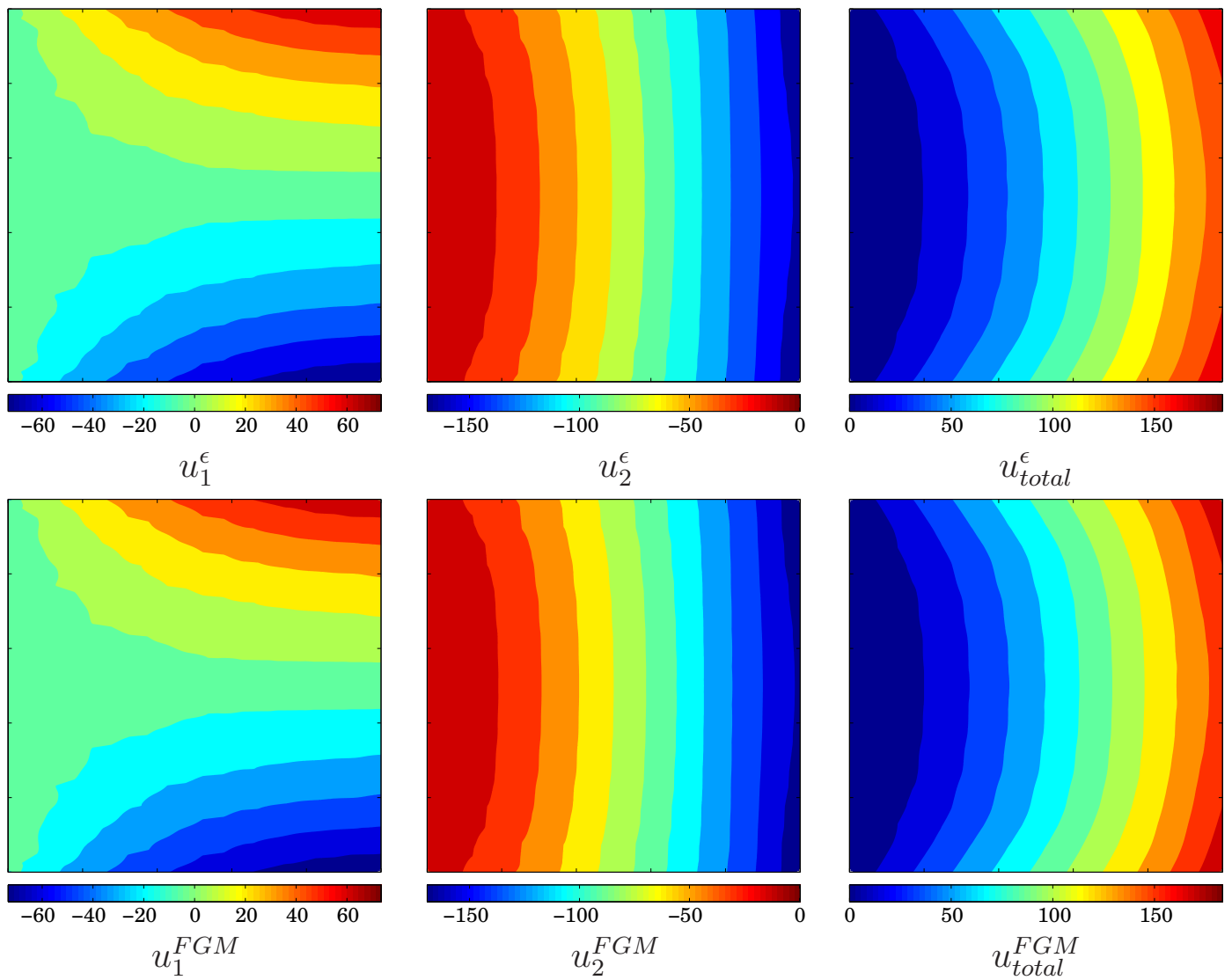

Figure 4.7: Total displacements calculated with full geometric model and homogenized model. 

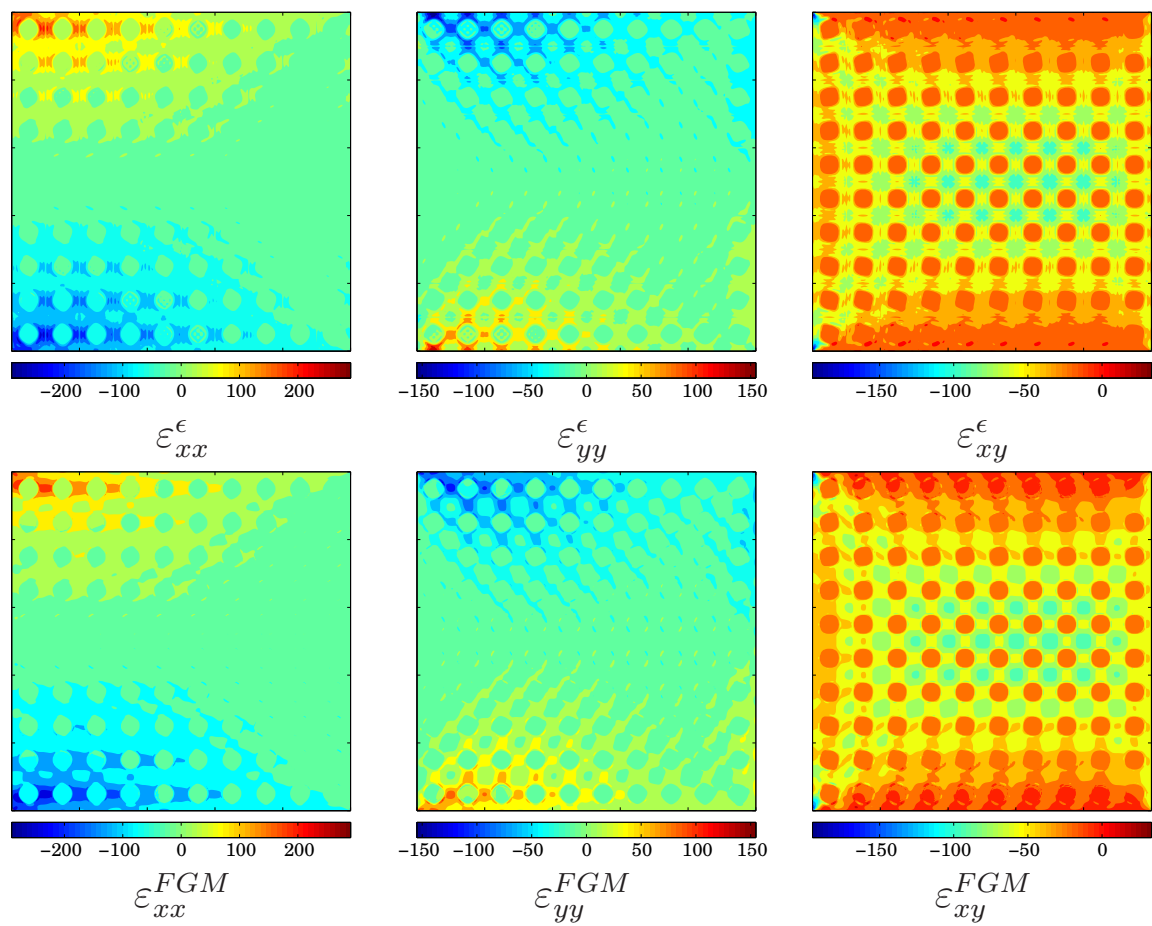

Figure 4.8: Strains calculated with full geometric model and homogenized model.
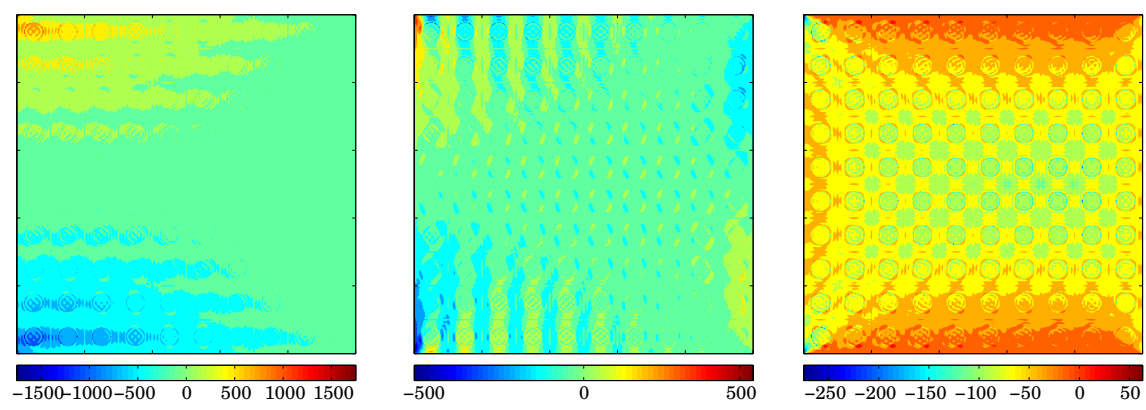

$$
\sigma_{x x}^{\epsilon}
$$

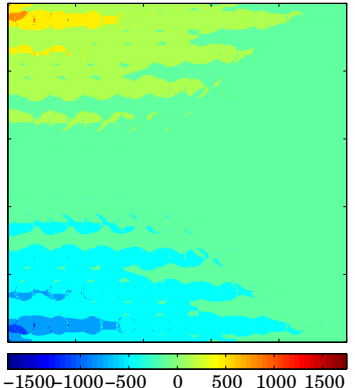

$$
\sigma_{x x}^{F G M}
$$
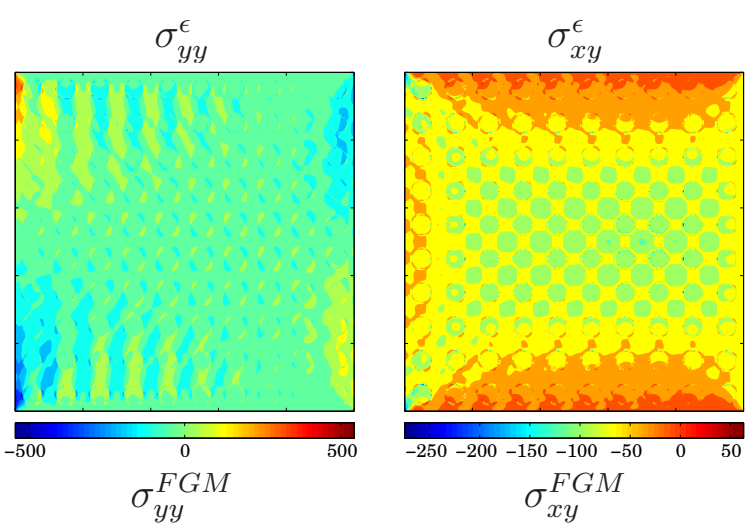

Figure 4.9: Stresses calculated with full geometric model and homogenized model. 


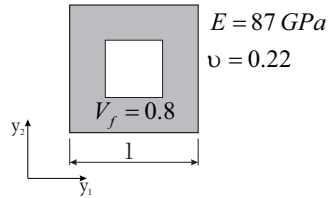

(a)

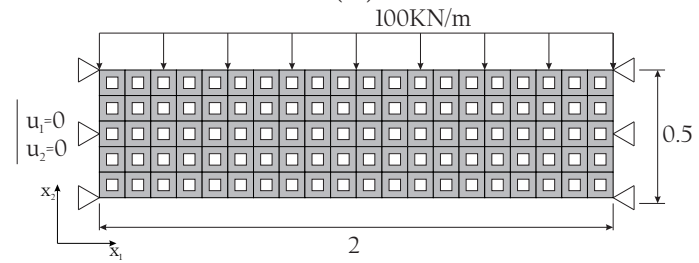

(c)

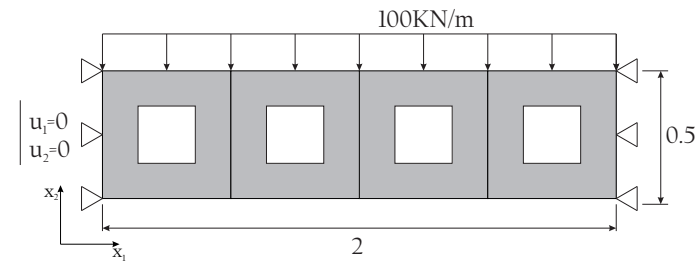

(b)

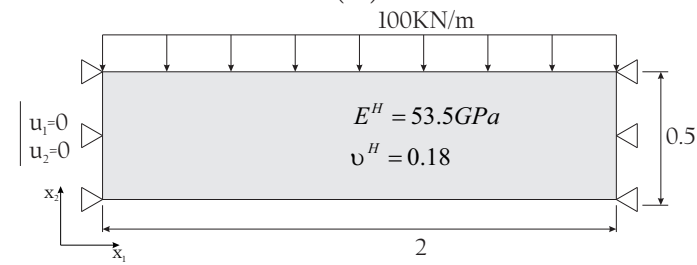

(d)

Figure 4.10: Geometry modeling (a) RVE; (b) Global domain with a $1 \times 4$ grid and boundary conditions ; (c) Global domain with a $5 \times 20$ grid and boundary conditions(d) Homogenized problem.

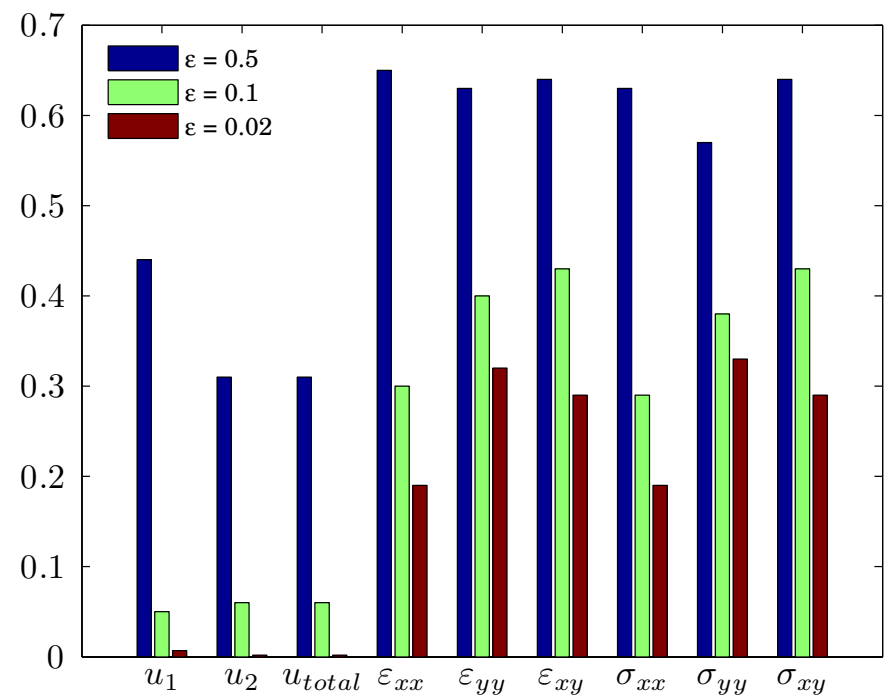

Figure 4.11: $L_{2}$-norms calculated for strains and stresses with different values of $\epsilon$. 

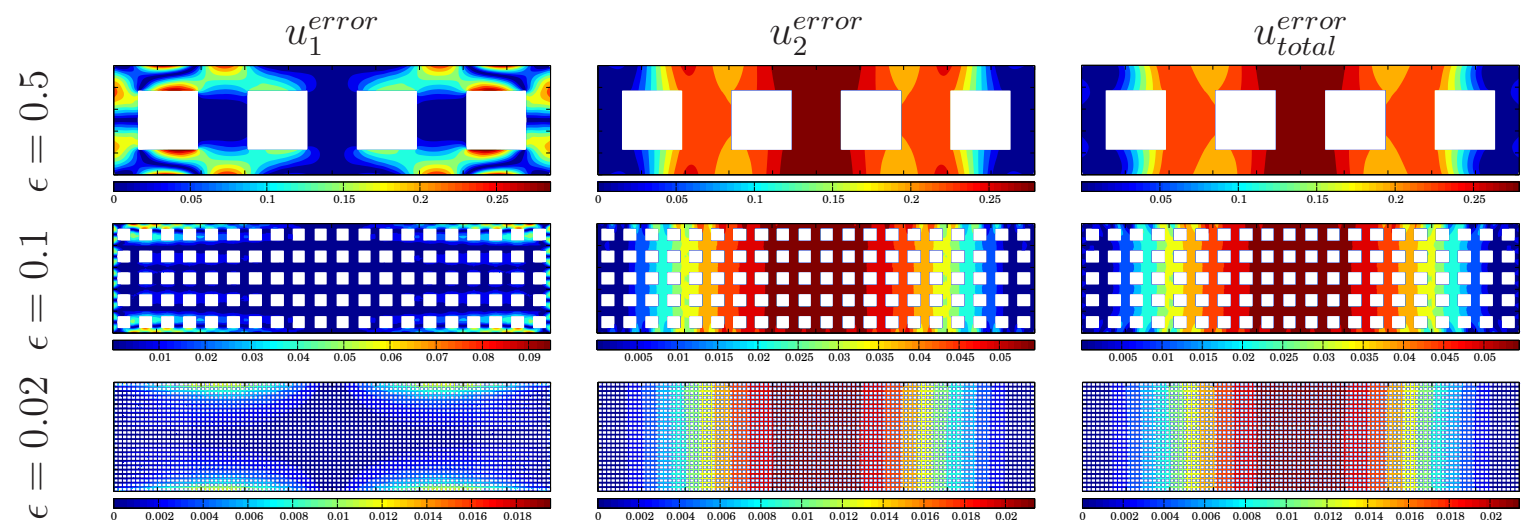

Figure 4.12: Error distribution for displacement over the global domains with $\epsilon=$ $0.5,0.1,0.02$.

presented in Figs. 4.12, 4.13 and 4.14.

Same as before error distribution plots for displacements shows very small error values and in plots corresponding to strains and stresses maximum values of error are limited to areas containing boundary cells. Based on $L_{2}$-norm and error distribution plots it is safe to say that, as expected when $\epsilon \rightarrow 0^{+}$the results of homogenized model converges to full geometric model.

In order to investigate the performance of proposed platform in predicting locations of maximum strains and stresses, results obtained from the homogenized model of the global domain with $\epsilon=0.1$ are compared with full geometric model and their plots are compared side by side in Figs. 4.15, 4.16 and 4.17.

\subsubsection{Spark plasma sintered TaC}

As the last numerical experiment carried out to evaluate the performance of meshfree/asymptotic platform in finding the local distribution of stresses and strains, the RVE shown in Fig. 3.24 is used to construct a global domain. Same as the previous two examples, RVEs are put together in three different $2 \times 2,10 \times 10$ and 

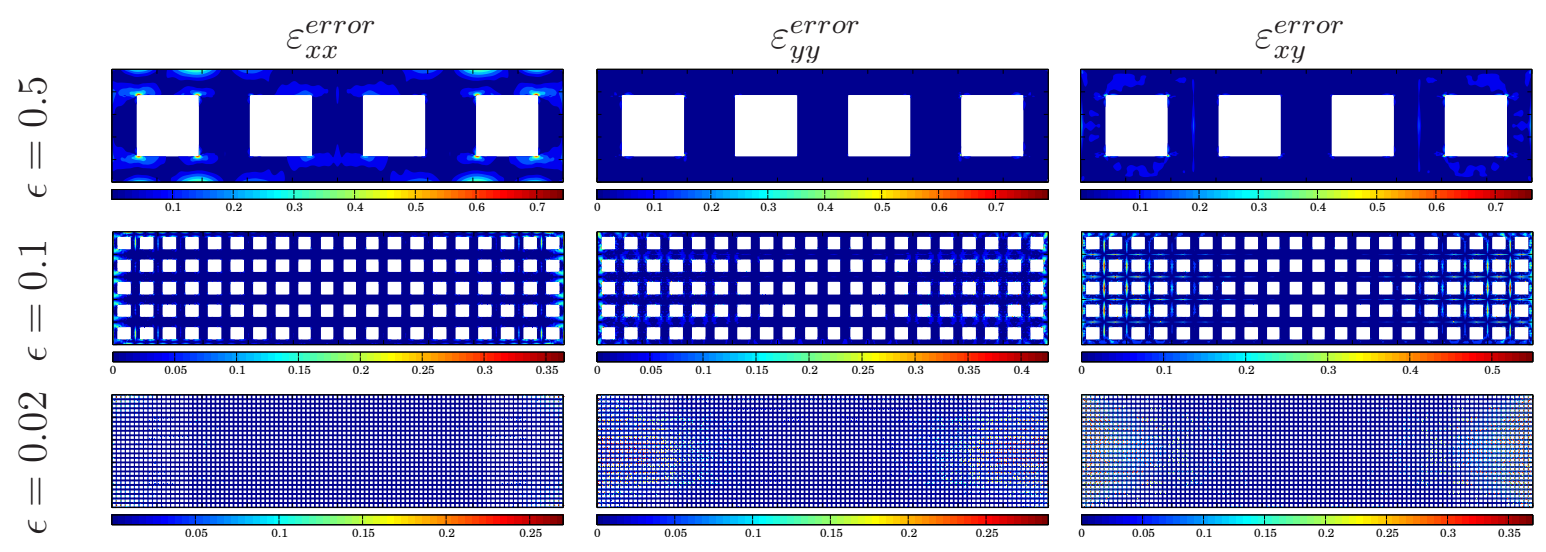

Figure 4.13: Error distribution for strains over the global domains with $\epsilon=$ $0.5,0.1,0.02$.
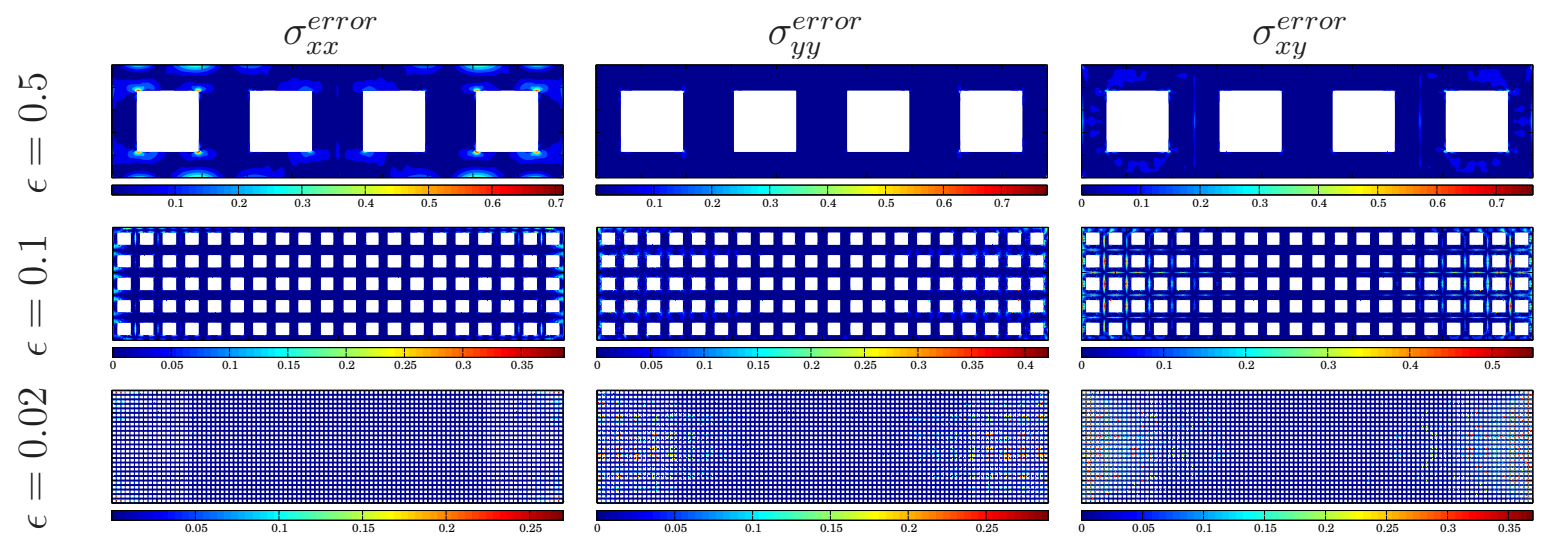

Figure 4.14: Error distribution for stresses over the global domains with $\epsilon=$ $0.5,0.1,0.02$.
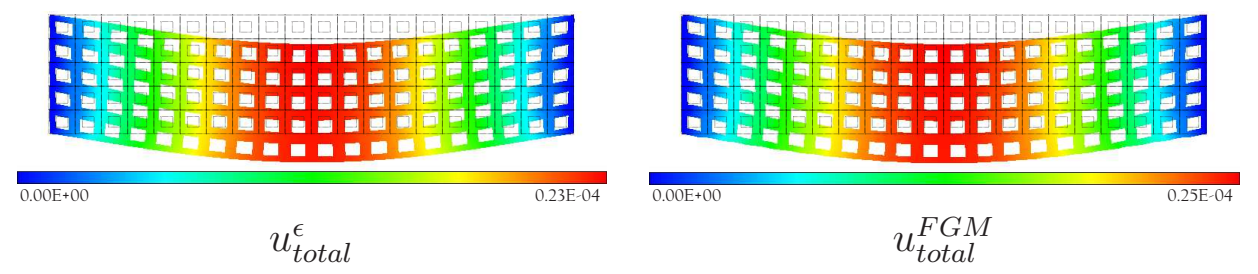

Figure 4.15: Total displacements calculated with full geometric model and homogenized model for a global domain constructed from square RVEs with a square void and $\epsilon=0.1$. 


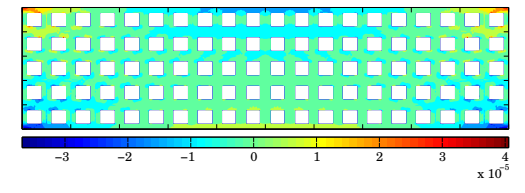

$\varepsilon_{x x}^{\epsilon}$

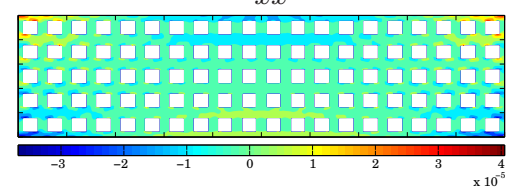

$\varepsilon_{x x}^{F G M}$

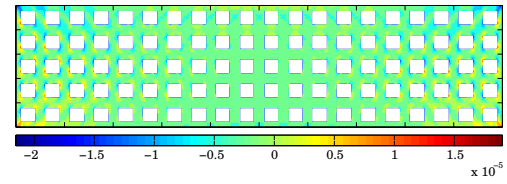

$\varepsilon_{y y}^{\epsilon}$

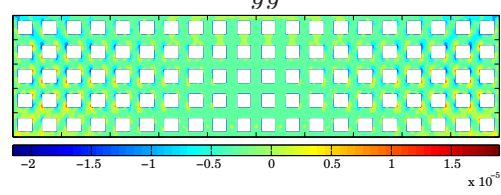

$\varepsilon_{y y}^{F G M}$

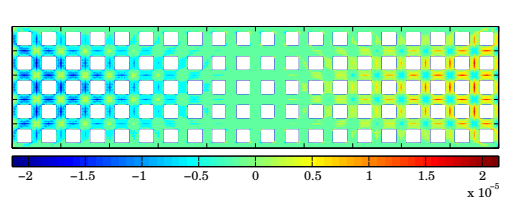

$\varepsilon_{x y}^{\epsilon}$

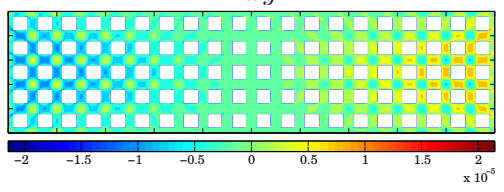

$\varepsilon_{x y}^{F G M}$

Figure 4.16: Strains and corresponding error distributions for a global domain constructed from square RVEs with a square void and $\epsilon=0.1$.

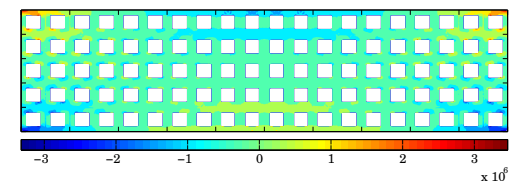

$\sigma_{x x}^{\epsilon}$

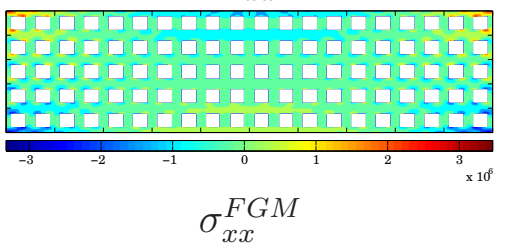

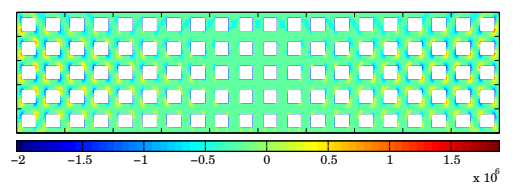

$$
\sigma_{y y}^{\epsilon}
$$

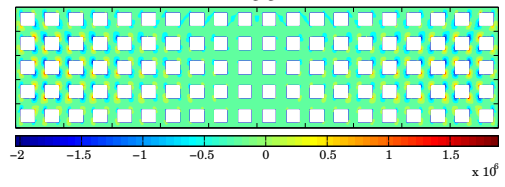

$\sigma_{y y}^{F G M}$

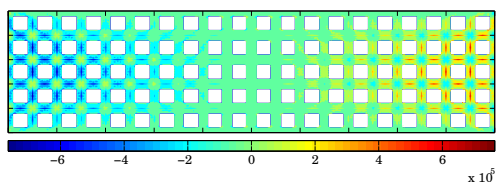

$\sigma_{x y}^{\epsilon}$

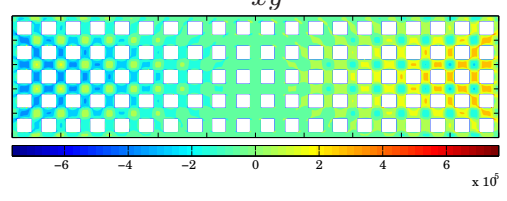

$\sigma_{x y}^{F G M}$

Figure 4.17: Stresses and corresponding error distributions for a global domain constructed from square RVEs with a square void and $\epsilon=0.1$. 


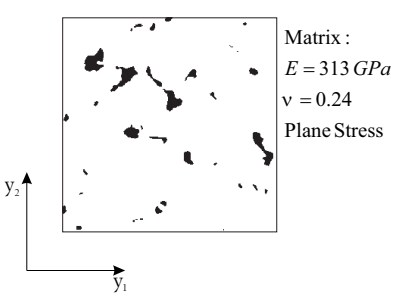

(a)

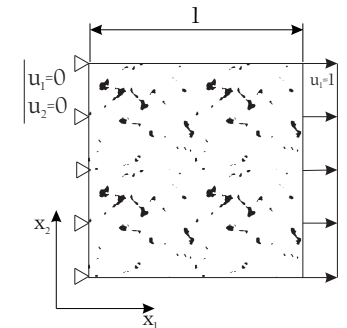

(b)

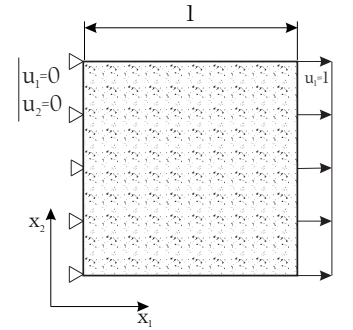

(c)

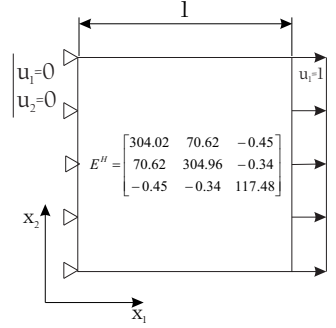

(d)

Figure 4.18: Geometry modeling (a) RVE; (b) Global domain with a $2 \times 2$ grid and boundary conditions ; (c) Global domain with a $10 \times 10$ grid and boundary conditions(d) Homogenized problem.

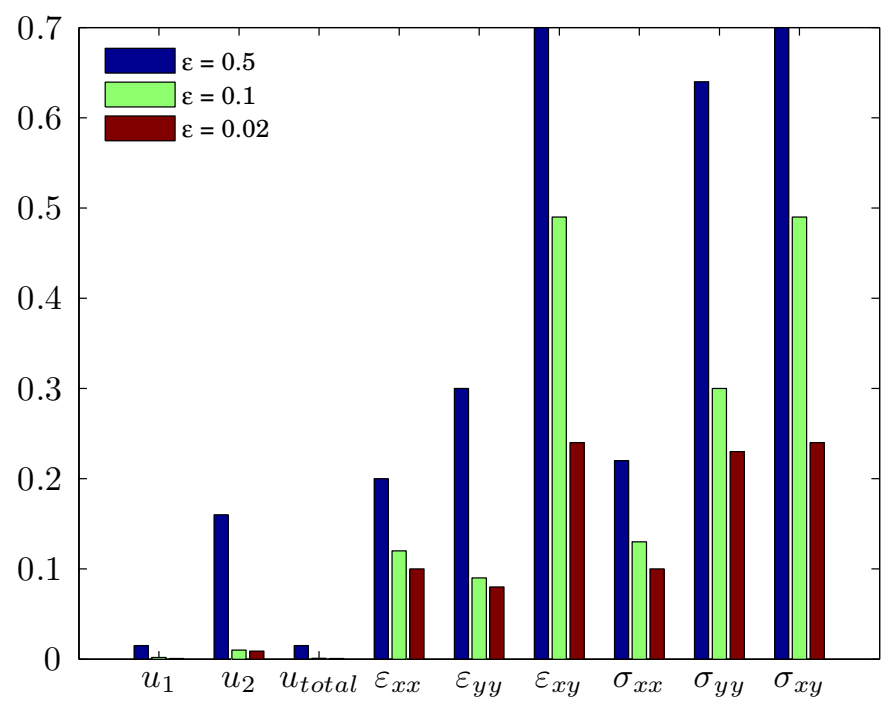

Figure 4.19: $L_{2}$-norms calculated for strains and stresses with different values of $\epsilon$.

$50 \times 50$ grids. Fig. 4.18(a) shows the RVE geometry and material properties, global domains created by $2 \times 2$ and $10 \times 10$ grids and corresponding boundary conditions are presented in Figs. 4.18(b) and (c) and Fig. 4.18(d) shows the homogenized problem setup.

Homogenized and full geometric models are solved for all three global domains and corresponding $L_{2}$-norms for displacements, strains and stresses are computed and presented in Fig. 4.19. Same as before, following the behaviour of $L_{2}$-norms 
clearly shows that as $\epsilon \rightarrow 0^{+}$results of homogenized solution converge toward the ones from full geometric model. Error distributions for displacements, strains and stresses are respectively presented in Figs. 4.20, 4.21 and 4.22 and Figs. 4.23 and 4.24 compare the values of strains and stresses calculated with homogenized and full geometric models side by side. 

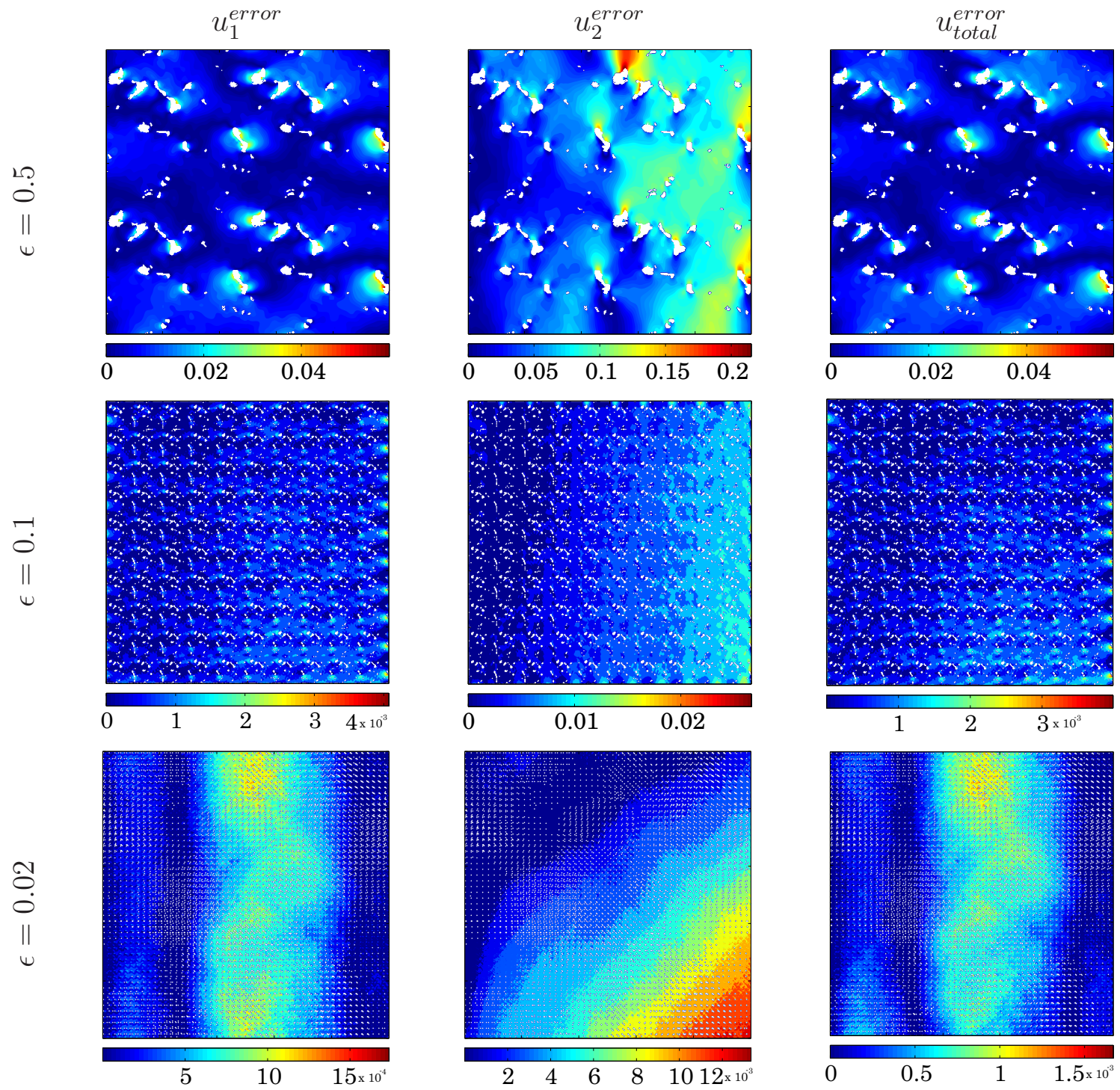

Figure 4.20: Error distribution for displacement over the global domains with $\epsilon=$ $0.5,0.1,0.02$. 

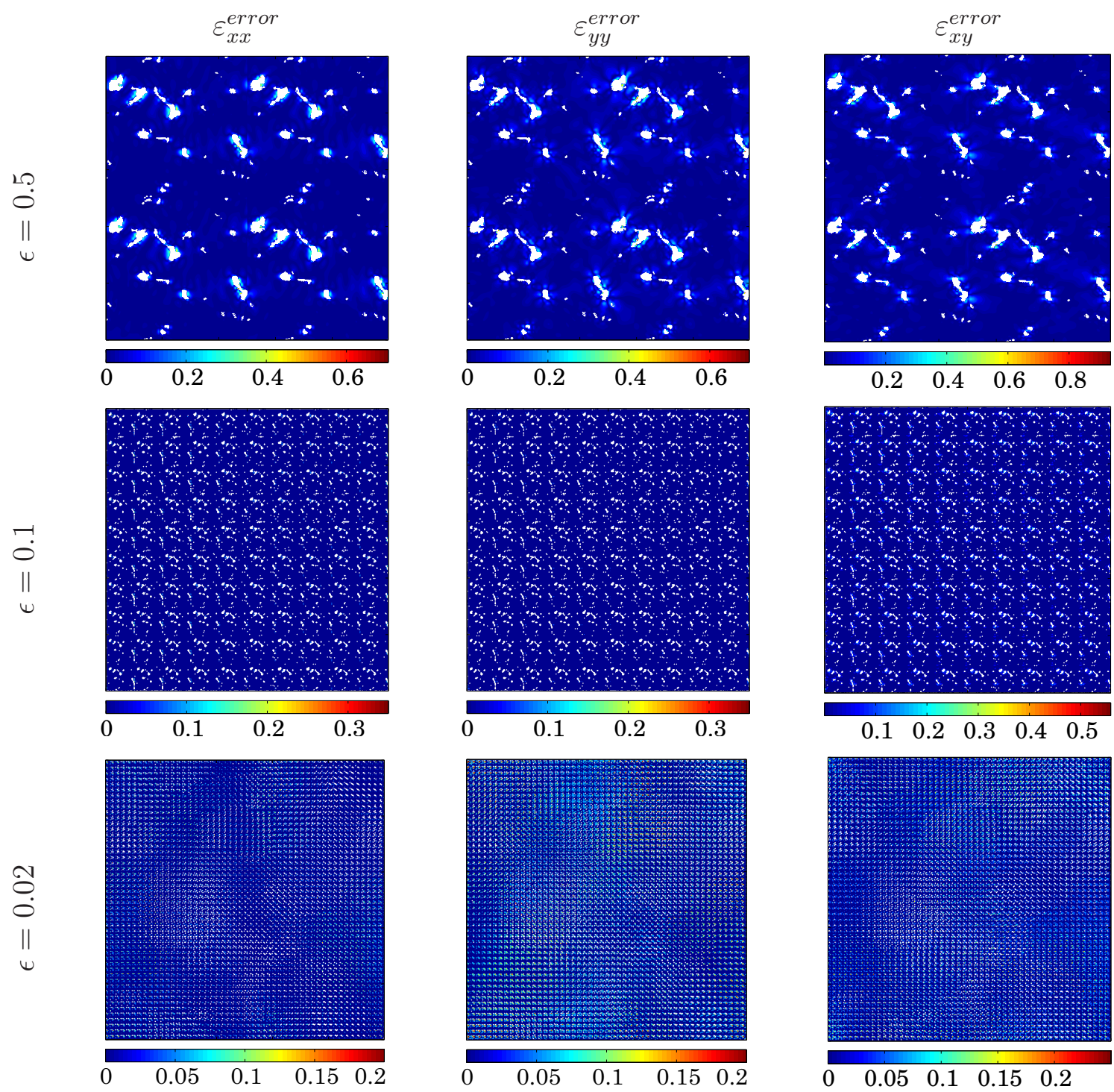

Figure 4.21: Error distribution for strains over the global domains with $\epsilon=$ $0.5,0.1,0.02$. 

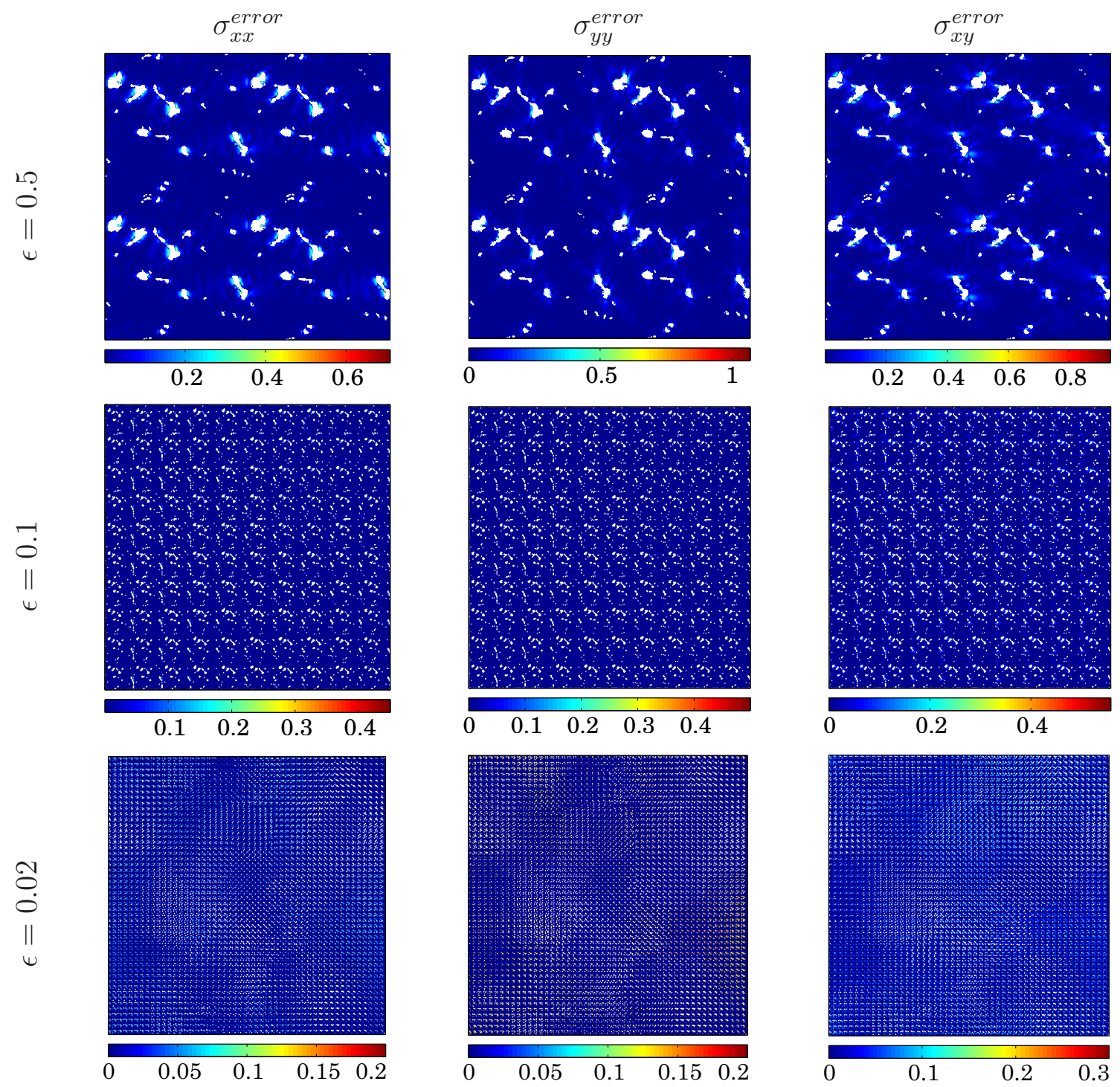

Figure 4.22: Error distribution for stresses over the global domains with $\epsilon=$ $0.5,0.1,0.02$. 

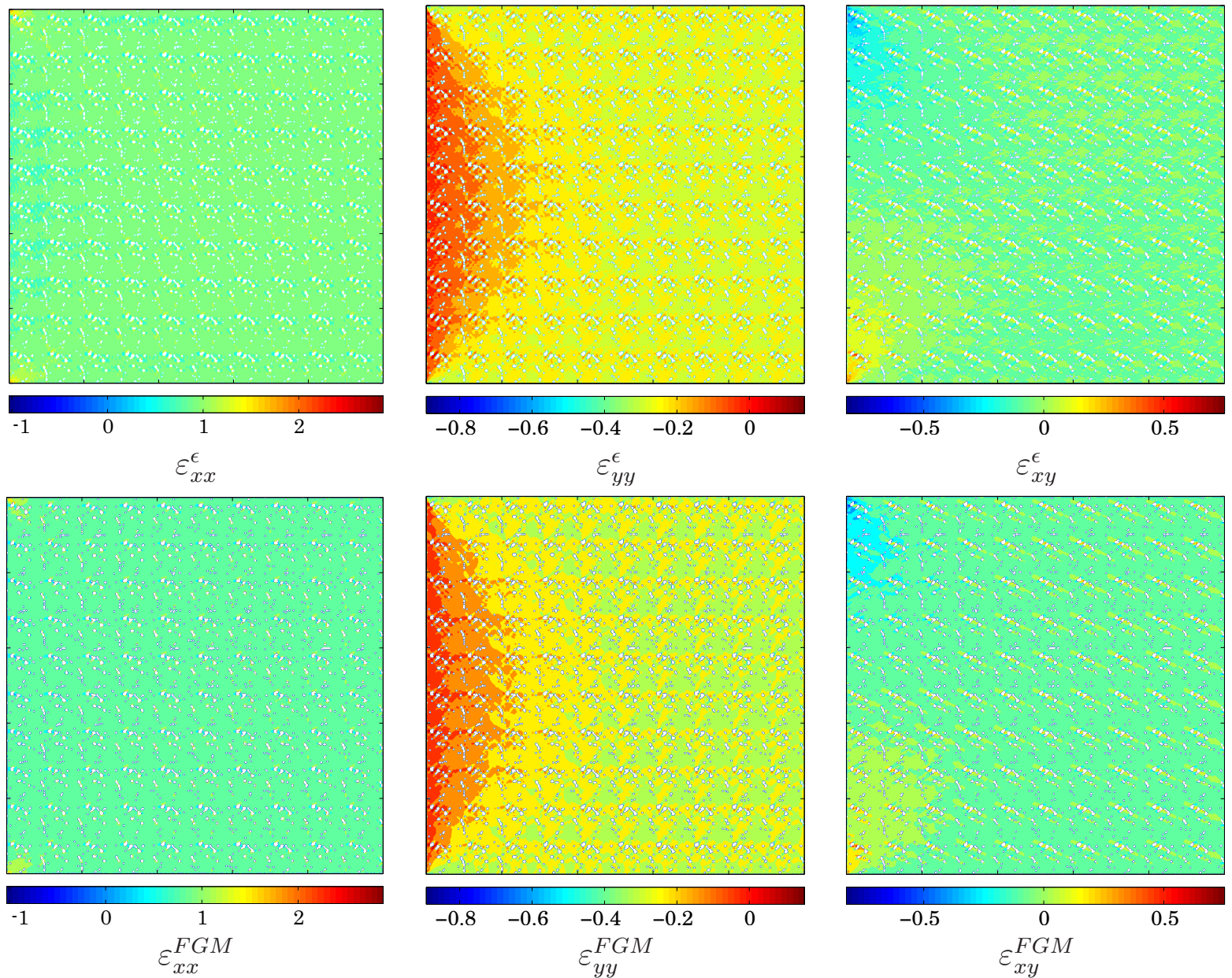

Figure 4.23: Strains and corresponding error distributions for a global domain constructed from square RVEs with a square void and $\epsilon=0.1$. 

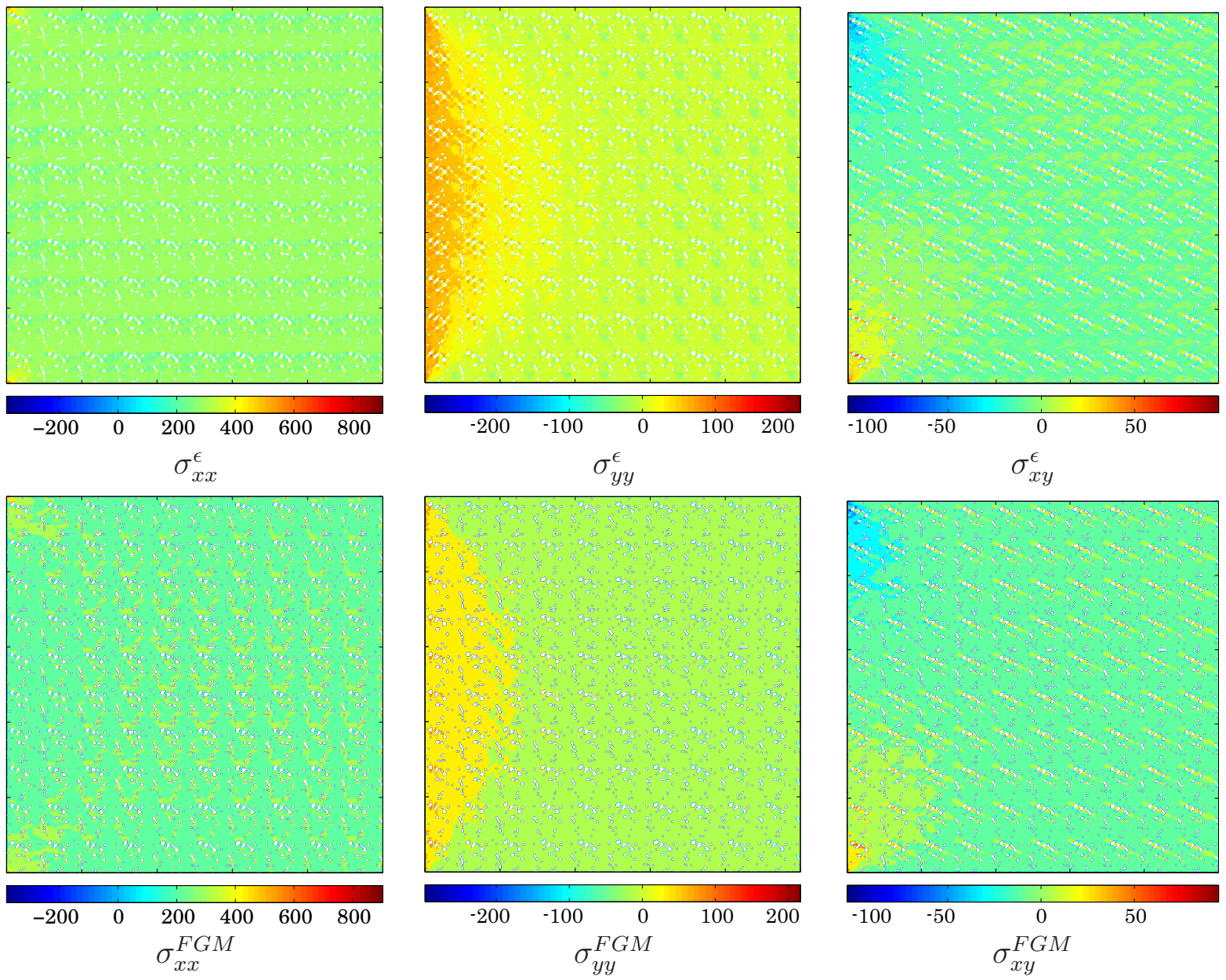

Figure 4.24: Stresses and corresponding error distributions for a global domain constructed from square RVEs with a square void and $\epsilon=0.1$. 


\section{CHAPTER 5 \\ EFFECTS OF GEOMETRIC FEATURES ON MECHANICAL PROPERTIES}

In Chapters 3 and 4 details of the developed computational platform were discussed. It was shown that the meshfree/asymptotic homogenization method can easily handle complex geometries. Also due to its meshfree nature, the mesh generation procedure can be completely removed and we can have access to a fully automated analysis.

In most of common homogenization methods, the only factor effecting homogenized properties is the volume fraction of inhomogeneities and the output effective elasticity tensor is always isotropic. But in the developed platform, in addition to volume fractions, spatial distribution of pores and inclusions is also taken into consideration, besides when dealing with nonsymmetric geometries or materials this platform is very well capable of predicting anisotropic effective properties.

In this chapter, we study RVEs with various distributions of pores or inclusions and investigate the effects of geometry on homogenized properties. Numerical experiments are designed in such way that in addition to different volume fractions, they can provide us several spatial distributions of inhomogeneities.

For example in Fig. 5.1 we have a unit length square shaped RVE with a rectangular $0.7 \times 0.3$ area marked and centered in it. This area is used as the permissible area for distribution of inhomogeneities. In this particular example 250 circular pores with the diameter of 0.02 are randomly distributed in the mentioned area. Then this distribution is rotated $180^{\circ} \mathrm{CCW}$ and the homogenized properties of the RVE are calculated every $5^{\circ}$. As shown in Figs. 5.1 (a) and (b), the volume fraction of pores are the same for every arrangement $\left(V_{f}=15.8 \%\right)$. Fig. 5.2 shows elasticity tensor members as a function of rotation angle, to calculate these values 


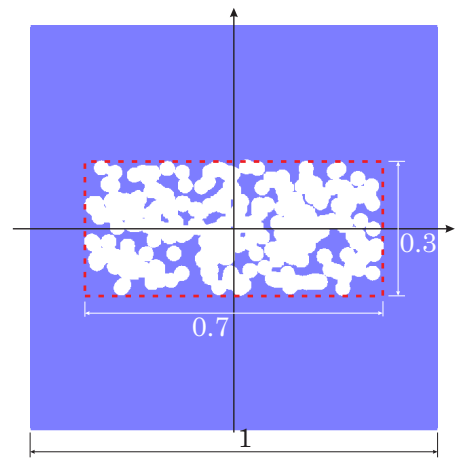

(a)

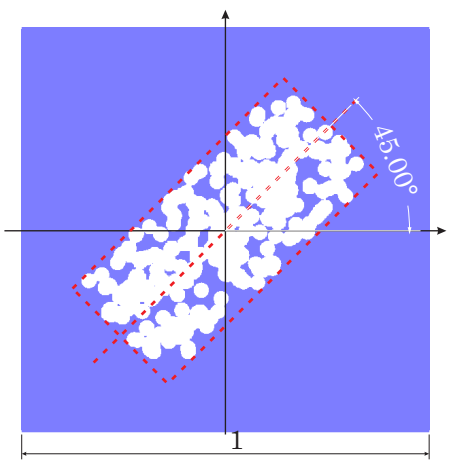

(b)

Figure 5.1: A unit length RVE with random distribution of circular porosities inside. Pores are distributed in a rectangular $0.7 \times 0.3$ area centered in the RVE with edges (a) parallel to $x$ and $y$ axis, (b) with a $45^{\circ} \mathrm{CCW}$ rotation.

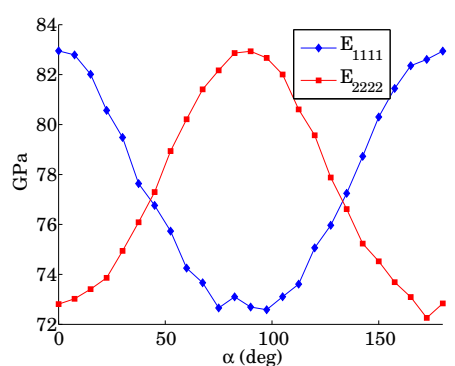

(a)

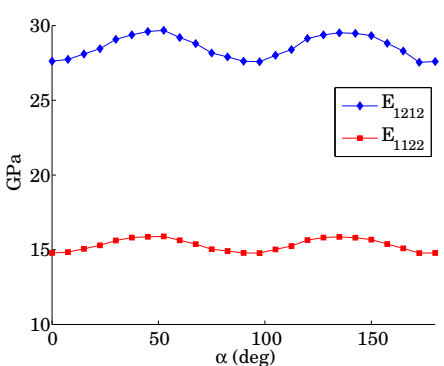

(b)

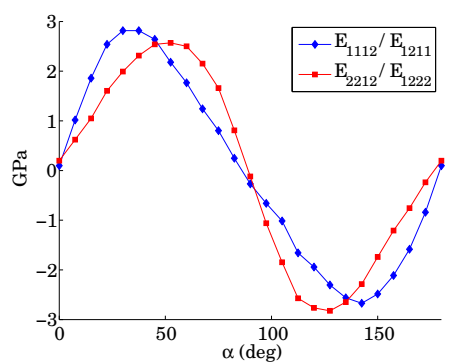

(c)

Figure 5.2: Elasticity tensor members as a function of rotation angle, (a) $E_{1111}$ and $E_{2222}$, (b) $E_{1212}$ and $E_{1122}$, (c) $E_{1112}$ and $E_{1211}$.

it is assumed that for the matrix material mechanical properties are $E=100 \mathrm{GPa}$, $\nu=0.2$ and the RVE is experiencing a plane stress loading. As shown in Fig. 5.2, although at all rotation angles the RVE has the same volume fraction of pores but the values of elasticity tensor members change considerably. Specially Fig. 5.2 (c), representing the changes in $E_{1112} / E_{1211}$ and $E_{2212} / E_{1222}$, clearly shows that most of the spatial arrangements will result in an anisotropic effective elasticity tensor, which other common homogenization techniques are not capable of recovering it.

Numerical experiments presented here can be categorized in three different groups 
based on the factor they study, those factors are: volume fraction, concentration of pores or inclusions and type of the basic geometry used to model inhomogeneities. In all experiments it is assumed that the properties for base material (matrix) are $E_{\text {matrix }}=100 \mathrm{GPa}$ and $\nu_{\text {matrix }}=0.2$. If the RVE contains pores, they are modeled as void spaces and if there is a second material mixed in the matrix, it is assumed that the second phase has the same Poisson's ratio as the matrix and it is ten times stronger which means $E_{\text {inclusion }}=1000 \mathrm{GPa}$ and $\nu_{\text {inclusion }}=0.2$.

\subsection{Effect of volume fraction}

To study the effect of volume fraction on effective mechanical properties, a square domain is considered as the RVE and it is filled with random number of reinforcing fibers. Fiber width is kept constant and equal to 0.0125, the lengthes are randomly sized within $[0.0,0.3]$ range and their orientation is also a random value between $[0, \pi]$. Fig. 5.3 shows ten different RVEs filled with fibers and the corresponding fiber volume fractions. Each RVE is homogenized and members of the resulting homogenized elasticity tensor are presented in Fig. 5.4.

As expected, an increase in the fiber volume fraction will increase the material stiffness and although the distributions are random but since they are almost evenly distributed around the RVE center, values of $E$ in $x$ and $y$ directions $\left(E_{1111}\right.$ and $\left.E_{2222}\right)$ are very close to each other. It can be observed that relation between values of elasticity tensor members and fiber volume fraction is a linear one. Comparing Figs. 5.4 (a) and (b) also shows that axial strength of the RVE is more sensitive to fiber volume fraction than shear strength. 


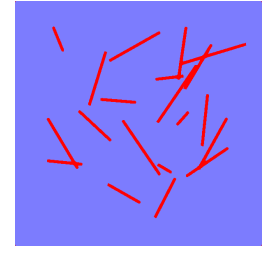

(a)

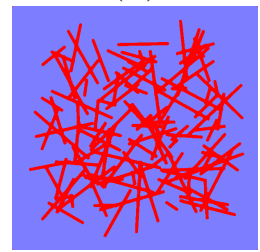

(f)

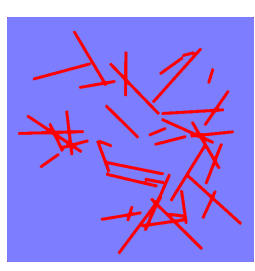

(b)

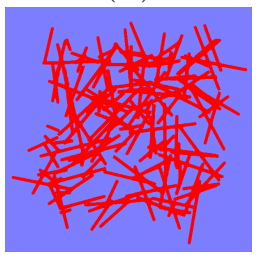

(g)

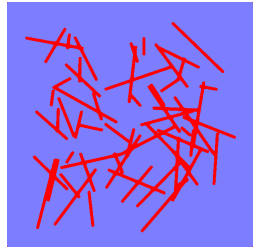

(c)

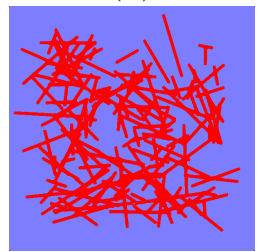

(h)

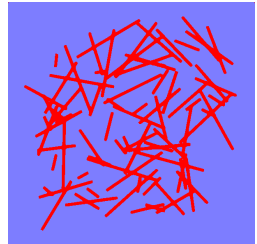

(d)

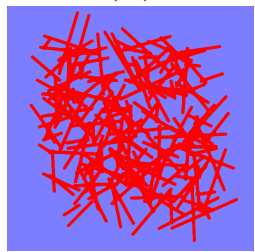

(i)

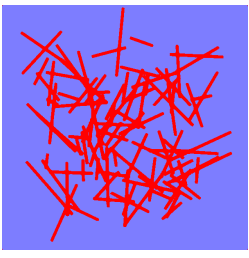

(e)

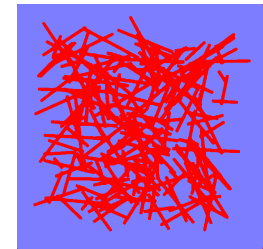

(j)

Figure 5.3: A unit length RVE with random distribution of different volume fractions of fibers. (a) $5 \%$, (b) $8 \%$, (c) $12 \%$, (d) $15 \%$, (e) $18 \%$, (f) $21 \%$, (g) $24 \%$, (h) $25 \%$, (i) $28 \%,(\mathrm{j}) 31 \%$.

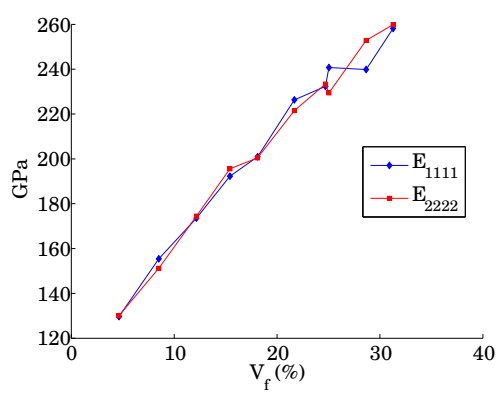

(a)

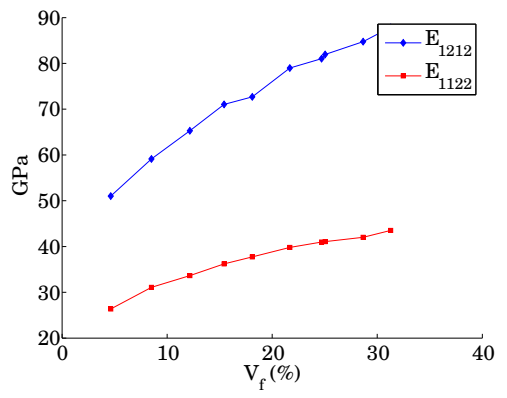

(b)

Figure 5.4: Elasticity tensor members as a function of fiber volume fraction, (a) $E_{1111}$ and $E_{2222}$, (b) $E_{1212}$ and $E_{1122}$. 


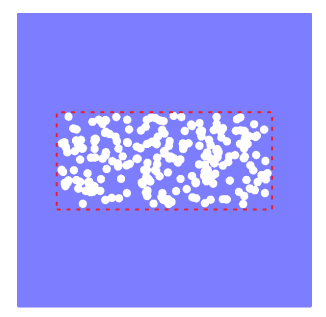

(a)

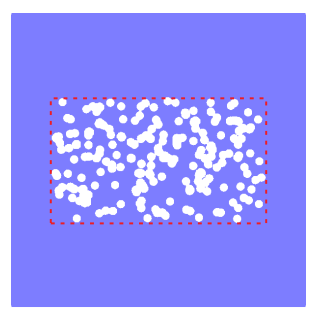

(b)

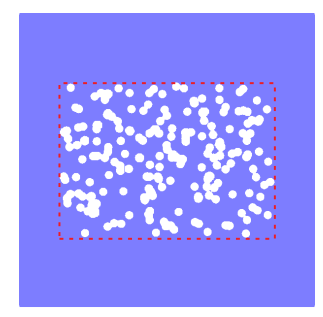

(c)

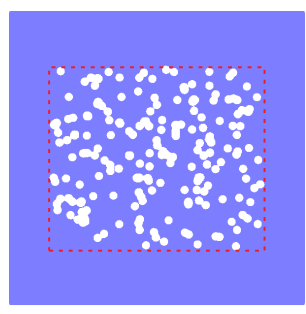

(d)

Figure 5.5: A unit length RVE with random distribution of circular porosities inside. Pores are distributed in a rectangular (a) $0.7 \times 0.3$, (b) $0.7 \times 0.4$, (c) $0.7 \times 0.5$, (d) $0.7 \times 0.6$ area.

\subsection{Effect of pore concentration}

In this numerical experiment, same square RVE is considered and four different rectangular areas are created in it as permissible areas for pore distribution. Size of these rectangular areas goes from $0.7 \times 0.3$ to $0.7 \times 0.6$ (width is kept constant and

heights change) and since number of pores are adjusted in such way that we always have a $10 \%$ volume fraction of pores, the smaller rectangles will lead to a more concentrated distribution of pores and the bigger ones give a wider distribution.

Fig. 5.5 shows the four different RVE arrangements, in each RVE the group of pores is rotated from $0^{\circ}$ to $180^{\circ}$ and at 24 different positions homogenized properties are calculated and recorded. Values of members of resulting homogenized elasticity tensors are plotted against rotation angles in Fig. 5.6.

Fig. 5.6 clearly shows that in addition to volume fraction and porosity orientation, the concentration of pores can also have a considerable effect on homogenized properties. All four RVEs have the same volume fraction of pores, but in all the plots in Fig. 5.6 the wider distribution of pores has led to a higher values of homogenized properties.

Recalling the equations for coordinate transfer of moment of inertia values, we know that if the moments of inertia for a given geometry are defined as $I_{x x}, I_{y y}$ and 


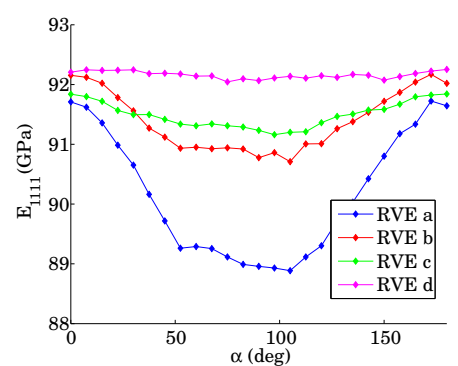

(a)

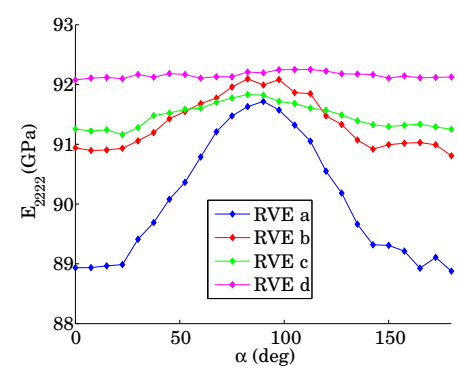

(b)

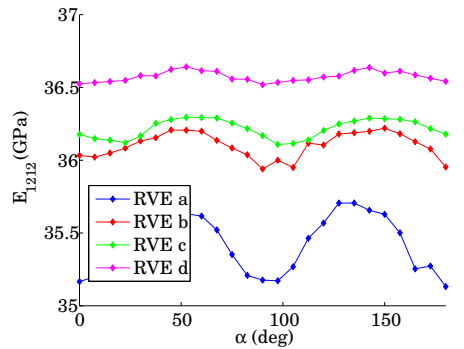

(c)

Figure 5.6: Elasticity tensor members as a function of rotation angle for different areas of pore distribution. (a) $E_{1111}$, (b) $E_{2222}$, (c) $E_{1212}$.

$I_{x y}$ in $x y$ coordinate system, the new values in $x y$ coordinate system which is the rotation of $x y$ by angle $\alpha$ will be:

$$
\begin{aligned}
& I_{\dot{x} \dot{x}}=\frac{I_{x x}+I_{y y}}{2}+\frac{I_{x x}-I_{y y}}{2} \cos (2 \alpha)-I_{x y} \sin (2 \alpha) \\
& I_{\dot{y} \dot{y}}=\frac{I_{x x}+I_{y y}}{2}+\frac{I_{x x}-I_{y y}}{2} \cos (2 \alpha)+I_{x y} \sin (2 \alpha) \\
& I_{\dot{x} \dot{y}}=\frac{I_{x x}-I_{y y}}{2} \sin (2 \alpha)+I_{x y} \operatorname{coas}(2 \alpha)
\end{aligned}
$$

Using this information and the fact that values of elasticity tensor members have a trigonometric behavior with respect to rotation angle, it can be predicted that the homogenized properties and the moment of inertia of pores would have a correlation as shown in Fig. 5.7.

In another experiment performed for investigating the effect of pore concentration on homogenized properties, the RVE presented in Fig. 5.5 (a) is used again. But instead of having a single $0.7 \times 0.3$ rectangle as permissible area, pores are distributed in four $0.35 \times 0.15$ rectangles centered in each one of the coordinate quadrants as shown in Fig. 5.8. Volume fraction of pores is kept at $10 \%$ and each rectangle is rotated from $0^{\circ}$ to $180^{\circ}$. Homogenized properties for the RVE with four separated pore distributions are computed and compared with the data for the RVE with a single permissible area for pore distribution in Fig. 5.9. 

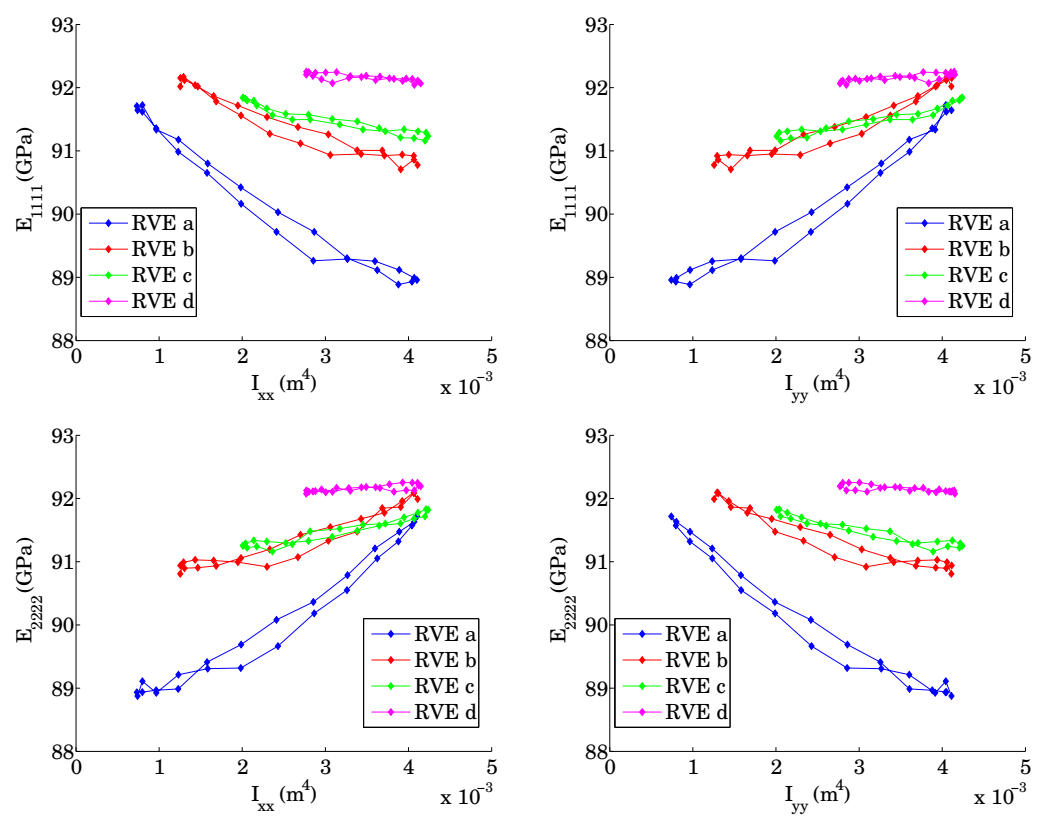

Figure 5.7: Elasticity tensor members as a function of moment of inertia for different areas of pore distribution.

As Fig. 5.9 shows, off-diagonal elements of homogenized elasticity tensor for both arrangement of pores are almost the same and although the diagonal elements of the RVE with four separate distribution of pores have higher values, but the differences are very small $(<1 \%)$.

Above experiments are repeated for new sets of RVEs which have the same geometric configurations as Figs. 5.5 and 5.8. The only difference is that instead of random distribution of circular pores inside the RVEs, they contain random distribution of inclusions of a second material stronger than matrix.

Figs. 5.10, 5.11 and 5.12 respectively show the RVE geometry, homogenized properties as a function of rotation angle and the correlation between homogenized properties and moments of inertia of the inclusions. Same as before, the plot presenting homogenized properties versus rotation angle shows that wider distribution of inclusions will lead to a higher values of homogenized properties in comparison 


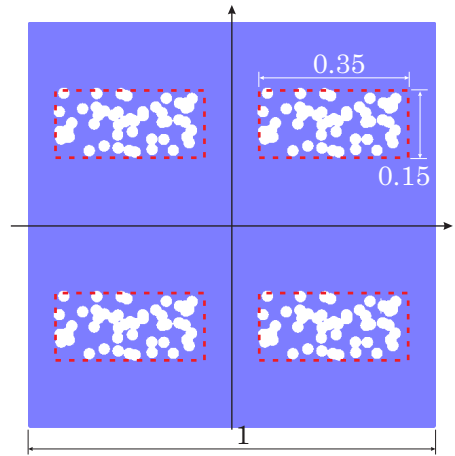

(a)

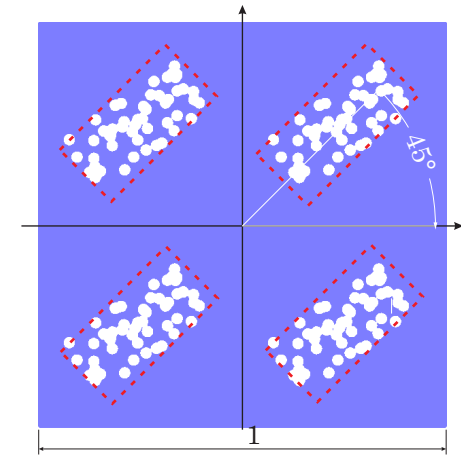

(b)

Figure 5.8: A unit length RVE with random distribution of circular porosities inside. Pores are distributed in 4 rectangular $0.35 \times 0.15$ areas with edges (a) parallel to $x$ and $y$ axis, (b) with a $45^{\circ} \mathrm{CCW}$ rotation.

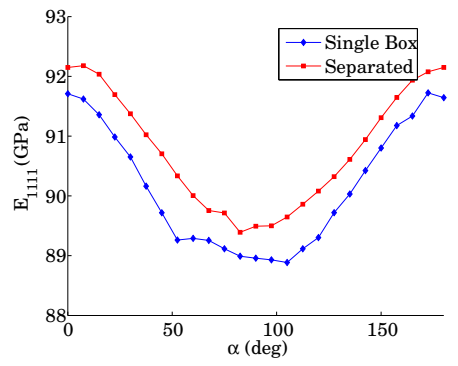

(a)

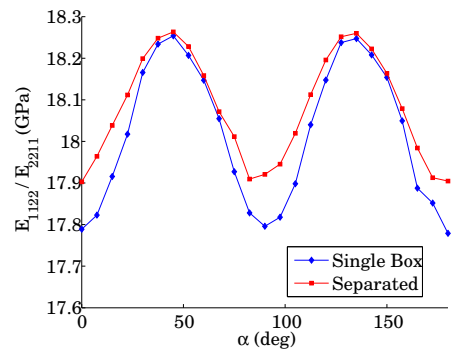

(d)

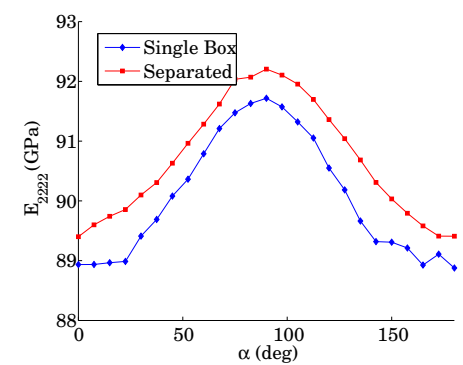

(b)

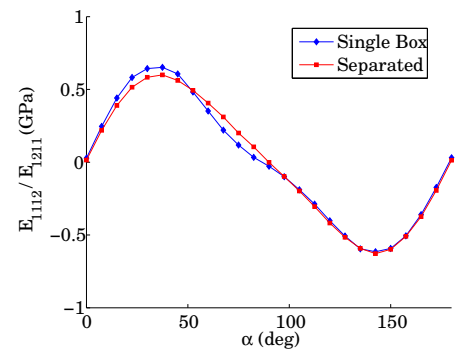

(e)

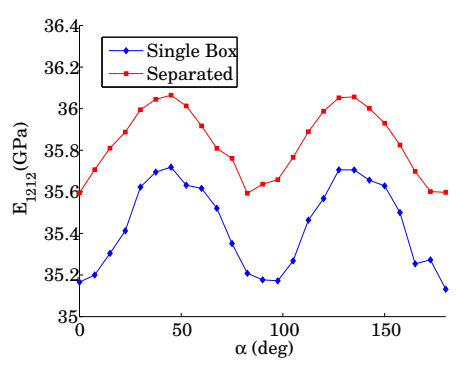

(c)

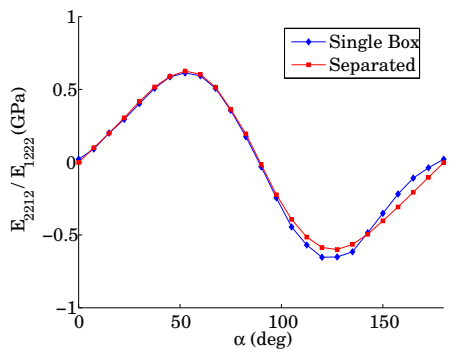

(f)

Figure 5.9: Elasticity tensor members as a function of rotation angle for pores distributed in a single rectangle and four separated ones. (a) $E_{1111}$, (b) $E_{2222}$, (c) $E_{1212}$, (d) $E_{1122}$, (e) $E_{1112}$, (c) $E_{2212}$. 


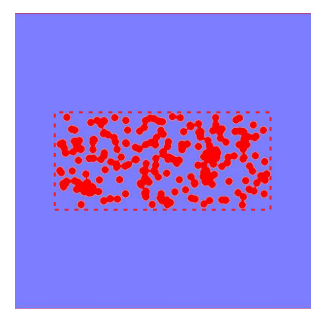

(a)

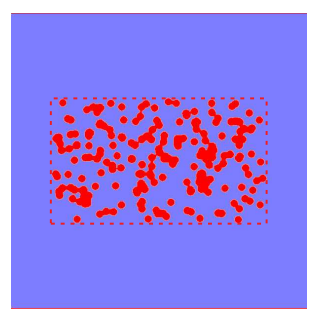

(b)

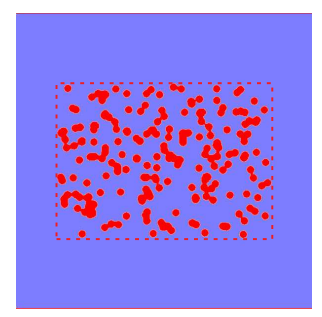

(c)

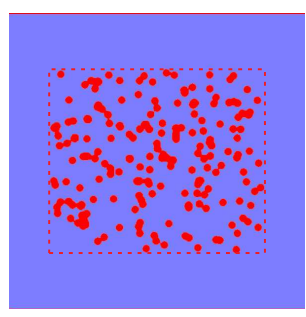

(d)

Figure 5.10: A unit length RVE with random distribution of a circular inclusions as reinforcement. Inclusions are distributed in a rectangular (a) $0.7 \times 0.3$, (b) $0.7 \times 0.4$, (c) $0.7 \times 0.5$, (d) $0.7 \times 0.6$ area.

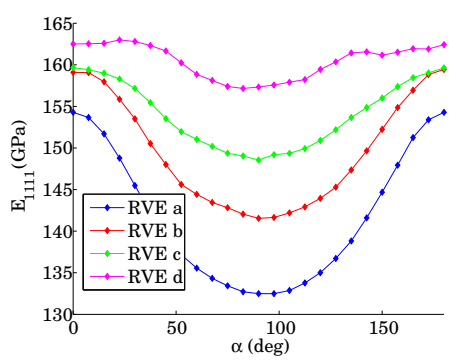

(a)

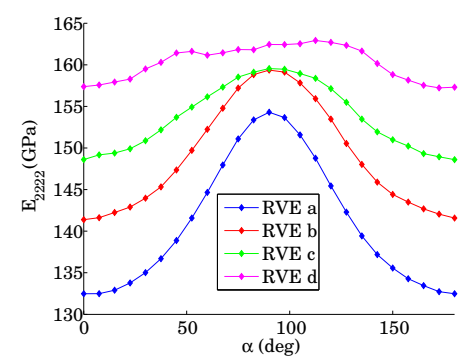

(b)

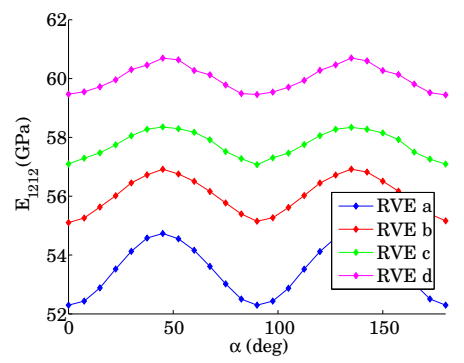

(c)

Figure 5.11: Elasticity tensor members of a RVE with two different materials as a function of rotation angle for different areas of pore distribution. (a) $E_{1111}$, (b) $E_{2222},(\mathrm{c}) E_{1212}$.

to a more concentrated distribution.

Plots comparing the homogenized properties of a RVE with a continuous distribution of inclusions to the one with four separated inclusion groups are presented in Fig. 5.13, which again shows the values of the off-diagonal elements are almost identical and the diagonal elements of the RVE with separated distribution of inclusions have a higher value, but the difference is very small $(<4 \%)$ 

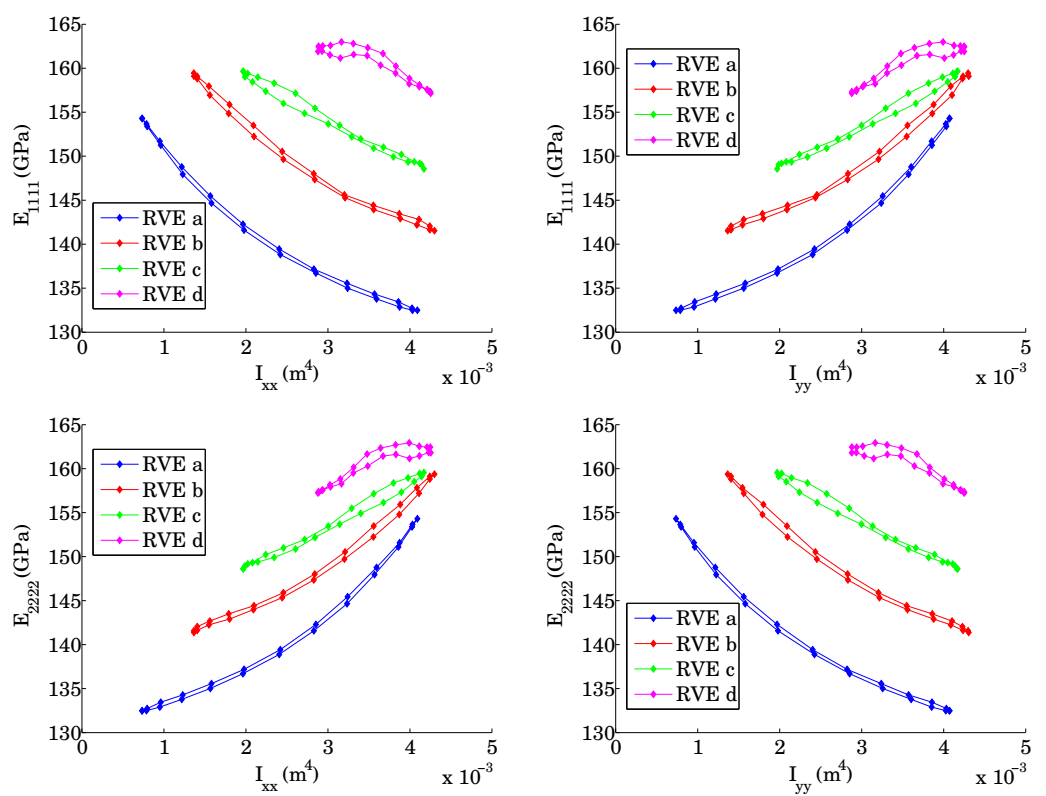

Figure 5.12: Elasticity tensor members as a function of moment of inertia for different areas of pore distribution.

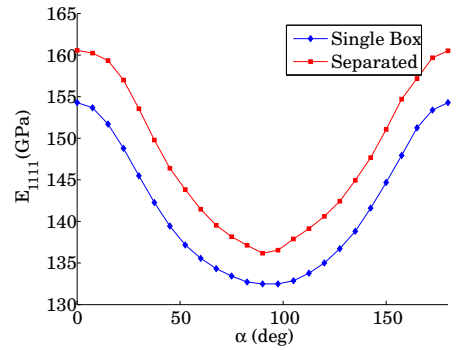

(a)

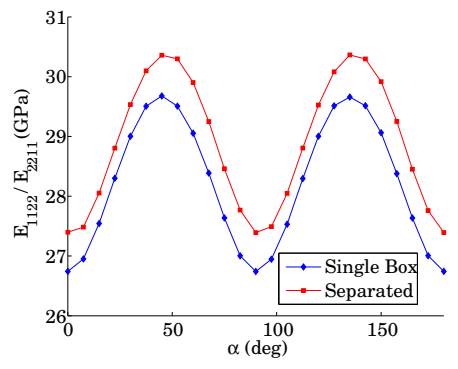

(d)

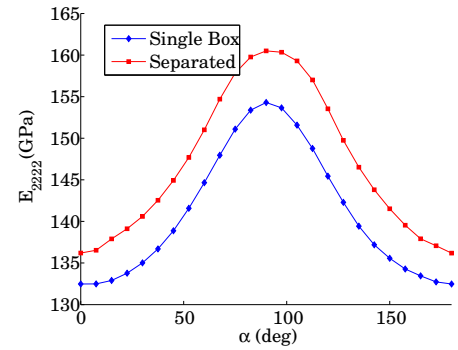

(b)

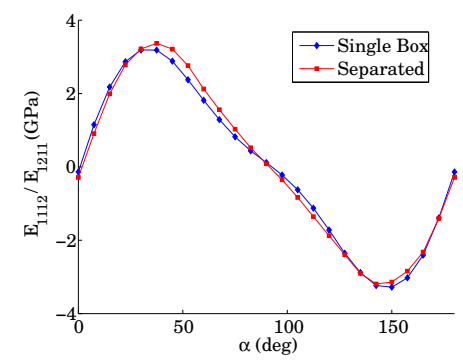

(e)

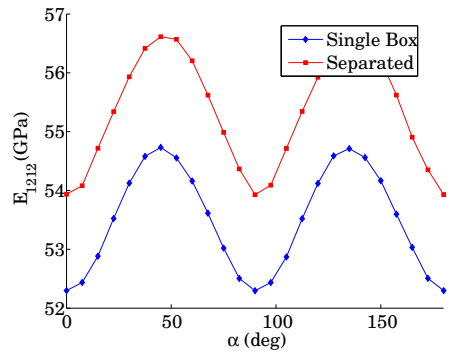

(c)

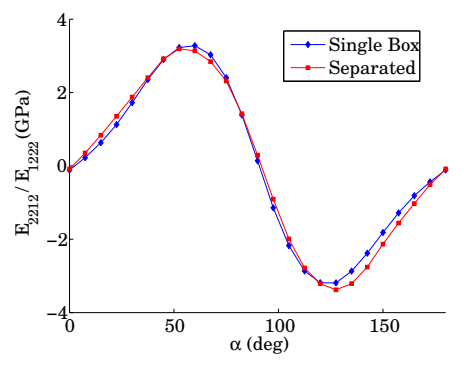

(f)

Figure 5.13: Elasticity tensor members as a function of rotation angle for inclusions distributed in a single rectangle and four separated ones. (a) $E_{1111}$, (b) $E_{2222}$, (c) $E_{1212}$, (d) $E_{1122}$, (e) $E_{1112}$, (c) $E_{2212}$. 


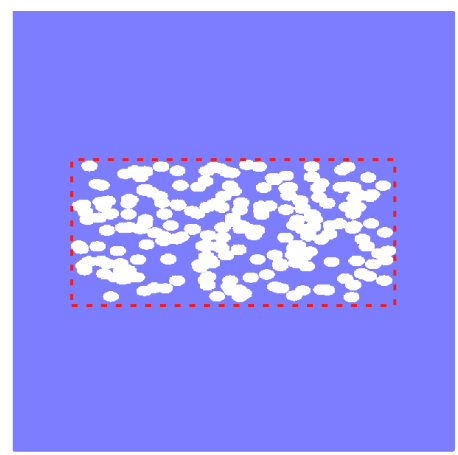

(a)

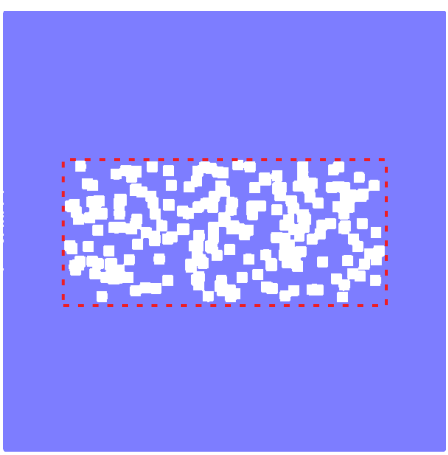

(b)

Figure 5.14: A unit length RVE with random distribution of pores inside. Pores are distributed in a $0.7 \times 0.3$ rectangular area and modeled with (a) ellipses, (b) Squares.

\subsection{Effect of pore shape}

In Figs. 5.5 (a) and 5.10 (a) pores and inclusions are modeled with circular shapes. Here same RVEs with the same permissible pore distribution areas are considered, but instead of using circles, pores are modeled with ellipses and squares as shown in Fig. 5.14. Same process has been repeated for the RVE containing inclusions of a stronger second material. The areas containing pores ad inclusions are rotated from $0^{\circ}$ to $180^{\circ}$ and homogenized properties are computed and recorded at 24 different positions.

Fig. 5.15 shows the recorded properties and compares them with properties of the RVE containing circular pores, as shown homogenized properties of this particular example does not depend that much on geometric shapes used to model pores. Fig. 5.16 represents the same properties for the RVEs containing inclusions. Again we can see that whether pores or inclusions are modeled with circles, ellipses or squares, computed homogenized properties are almost the same. 


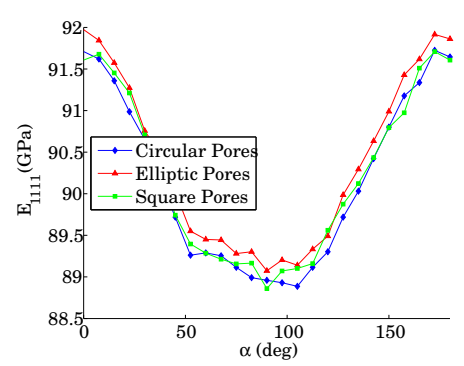

(a)

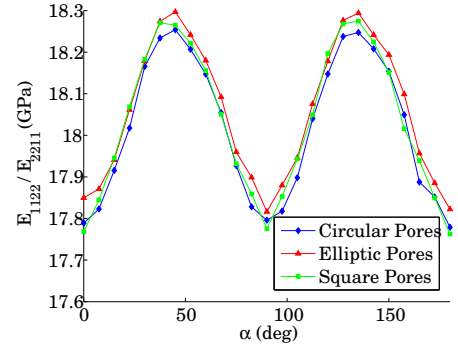

(d)

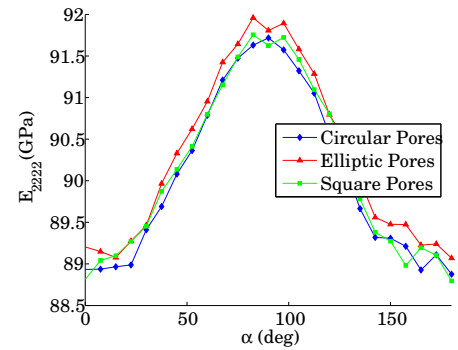

(b)

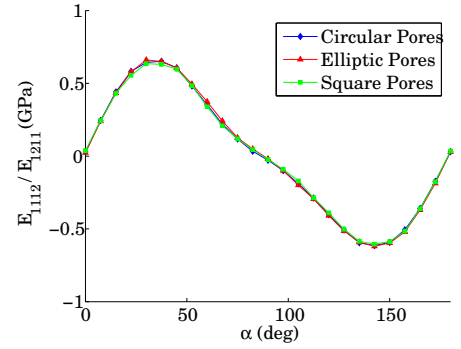

(e)

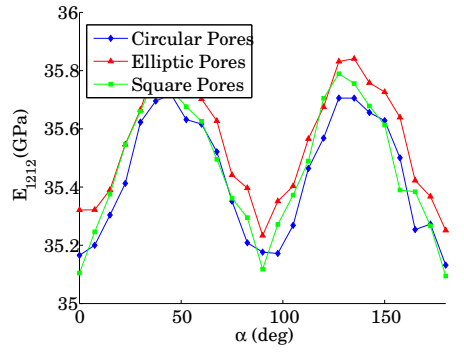

(c)

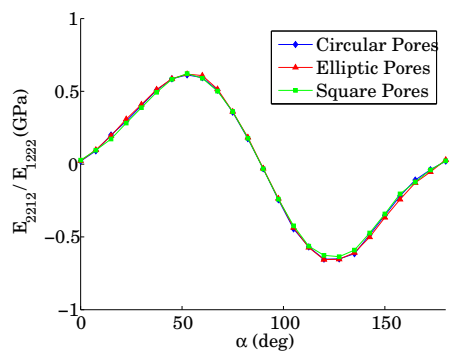

(f)

Figure 5.15: Elasticity tensor members as a function of rotation angle for pores modeled with circles, ellipses and squares. (a) $E_{1111}$, (b) $E_{2222}$, (c) $E_{1212}$, (d) $E_{1122}$, (e) $E_{1112}$, (c) $E_{2212}$.

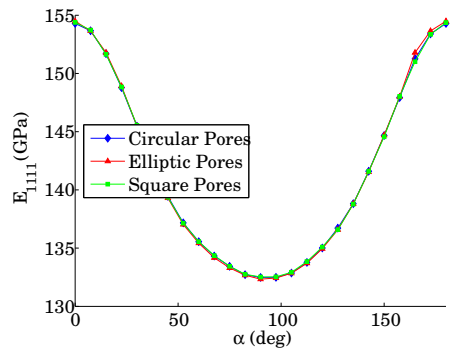

(a)

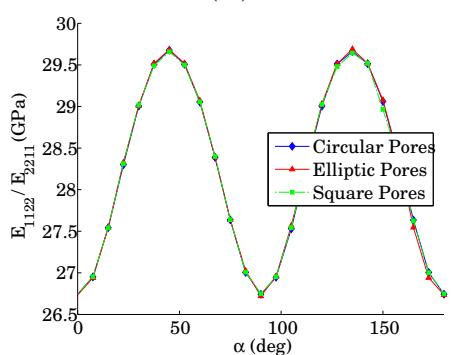

(d)

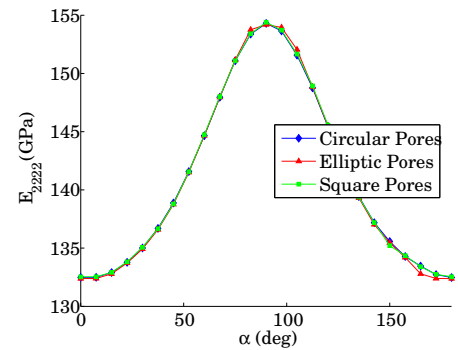

(b)

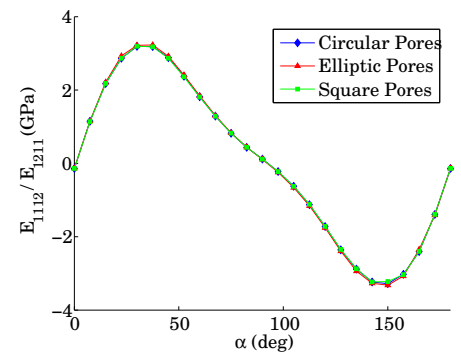

(e)

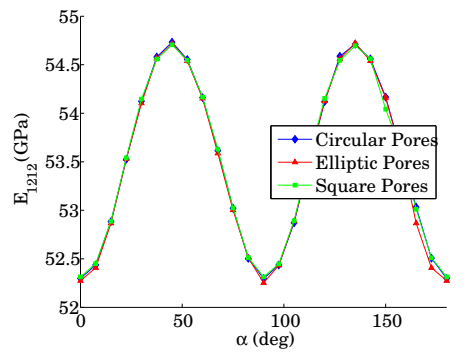

(c)

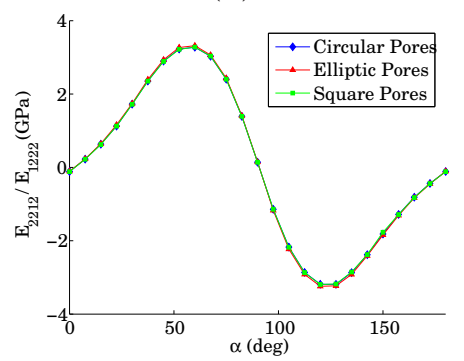

(f)

Figure 5.16: Elasticity tensor members as a function of rotation angle for inclusions modeled with circles, ellipses and squares. (a) $E_{1111}$, (b) $E_{2222}$, (c) $E_{1212}$, (d) $E_{1122}$, (e) $E_{1112},\left(\right.$ c) $E_{2212}$. 
CHAPTER 6

\section{HOMOGENIZATION OF THREE DIMENSIONAL DOMAINS}

The mathematical formulation derived for homogenization in Chapter 3 is the general tensor notation of equations and depending on the ranges of indices in eqs. (3.6) - (3.11) it can be implemented on 3D as well as 2D domains. For a three dimensional problem (when $i, j, k, l, m, n=1,2,3$ ) eq. (3.7) should be solved for six different sets of $k l=11,22,33,23 / 32,13 / 31,12 / 21$. Which will lead to six different sets of linear algebraic equations in the form of eq. (3.9). Since the elements of $[A]$ matrix do not depend on $k l$ values, the coefficient matrixes of all these linear equation sets are the same and the only thing changing is the $[b]$ vector. As

we know solving these systems will give us the values for $\left\{C_{q m}^{k l}\right\}$ and eventually $\left\{\chi_{m}^{k l}\right\}$ functions, which will be substituted in eq. (3.1) to calculate the homogenized properties.

Although the workflow diagram of developed homogenization platform for three dimensional problems is the same as Fig. 3.6, but changes need to be made specially in distance field generation and adaptive integration blocks to make them compatible with 3D domains. Details of theses modifications are extensively discussed in [39, $64,11]$.

In addition to above modifications, a new set of enrichment functions suitable for $3 \mathrm{D}$ domains has to be defined which can satisfy periodic boundary conditions on three dimensional RVEs, see eqs. (3.5) and (3.15). Enrichment functions implemented to ensure satisfaction of periodic boundary conditions over a cubic RVE are described in eq. (6.1).

Same as previous chapters, here a group of numerical experiments are chosen to evaluate the performance of meshfree/asymptotic homogenization platform and the 
results are compared with previously published data and/or strain energy method.

$$
\begin{aligned}
& \Delta x=x_{\max }-x_{\min } \quad \Delta y=y_{\max }-y_{\min } \quad \Delta z=z_{\max }-z_{\min } \\
& x_{c}=\frac{x_{\max }+x_{\min }}{2} \quad y_{c}=\frac{y_{\max }+y_{\min }}{2} \quad z_{c}=\frac{z_{\max }+z_{\min }}{2} \\
& \text { For } k=1 \quad \psi_{1}=\left[\left(\frac{\Delta y}{2}\right)^{2}-\left(y-y_{c}\right)^{2}\right]\left[\left(\frac{\Delta z}{2}\right)^{2}-\left(z-z_{c}\right)^{2}\right]\left(x-x_{c}\right)^{2} \\
& \psi_{2}=\left[\left(\frac{\Delta x}{2}\right)^{2}-\left(x-x_{c}\right)^{2}\right]\left[\left(\frac{\Delta z}{2}\right)^{2}-\left(z-z_{c}\right)^{2}\right]\left(y-y_{c}\right)^{2} \\
& \psi_{3}=\left[\left(\frac{\Delta y}{2}\right)^{2}-\left(y-y_{c}\right)^{2}\right]\left[\left(\frac{\Delta x}{2}\right)^{2}-\left(x-x_{c}\right)^{2}\right]\left(z-z_{c}\right)^{2} \\
& \text { For } k=2,3, \ldots \quad \psi_{3 k-2}=\left(x-x_{c}\right)^{2} \prod_{j=0}^{2^{k}}\left[y-\left(y_{\min }+\frac{j}{2^{k}} \Delta y\right)\right]\left[z-\left(z_{\min }+\frac{j}{2^{k}} \Delta z\right)\right] \\
& \psi_{3 k-1}=\left(y-y_{c}\right)^{2} \prod_{j=0}^{2^{k}}\left[x-\left(x_{\min }+\frac{j}{2^{k}} \Delta x\right)\right]\left[z-\left(z_{\min }+\frac{j}{2^{k}} \Delta z\right)\right] \\
& \psi_{3 k}=\left(z-z_{c}\right)^{2} \prod_{j=0}^{2^{k}}\left[y-\left(y_{\min }+\frac{j}{2^{k}} \Delta y\right)\right]\left[x-\left(x_{\min }+\frac{j}{2^{k}} \Delta x\right)\right]
\end{aligned}
$$

\subsection{Layered Composite}

As the first numerical experiment on 3D domains a simple layered composite is studied and as a heterogeneous material its homogenized mechanical properties are calculated. A representative volume element of the structure is shown in Fig. 6.1 and Table 6.1 contains corresponding material properties. Effective properties of mentioned structure was studied in [47] by using conventional finite element and here those results will be used to evaluate the performance of MeshFree/Asymptotic method on a 3D geometry.

Following the work flow presented in Chapter 3, first distance field functions are created for each phase in the structure, a nonconforming grid is laid over the domain and Gauss integration points are distributed. Fig. 6.2 shows the distance 


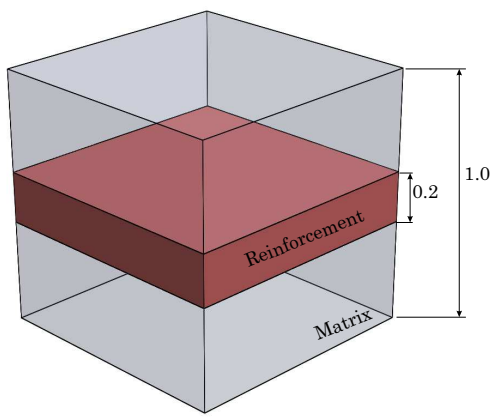

Figure 6.1: RVE geometry for a composite structure with layer reinforcement.

Table 6.1: Mechanical properties of matrix and reinforcement for the layered composite[47].

\begin{tabular}{ll}
\hline Property & Value \\
\hline Matrix elastic modulus, $E_{m}(\mathrm{GPa})$ & 3.5 \\
Matrix Poisson's ratio, $\nu_{m}$ & 0.35 \\
Reinforcement elastic modulus, $E_{m}(\mathrm{GPa})$ & 72.0 \\
Reinforcement Poisson's ratio, $\nu_{m}$ & 0.30 \\
\hline
\end{tabular}




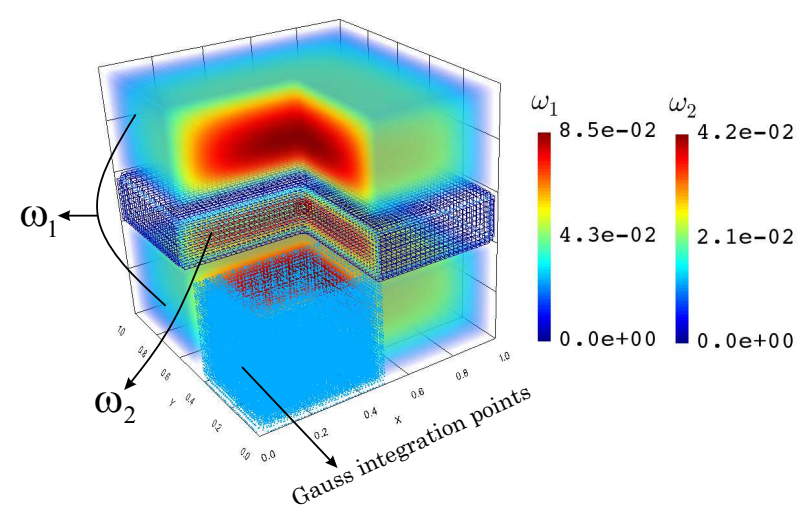

Figure 6.2: Distance field functions for matrix and reinforcement layer and Gauss integration point distribution in a section of domain.

field functions for matrix and reinforcement and also distribution of integration points are illustrated for a small section of the domain.

A solution structure similar to eq. (3.6) is created and 3D enrichment functions presented in eq. (6.1) are incorporated in it, and finally system of linear equations are solved and $\chi$ functions are computed. Fig. 6.3 shows the corresponding $\chi_{m}^{k l}$ functions for $m=1$ and homogenized properties are presented and compared with strain energy method in Table 6.2. Since the RVE shown in Fig. 6.1 has three planes of symmetry, behaviour of $\chi$ functions for $m=2,3$ are going to be very similar to the ones with $m=1$ and the only difference would be the maximum and minimum values of the functions. Therefor 6 out of total of $18 \chi$ functions are presented here for illustration purposes.

\subsection{Composite Matrix Reinforced with Boron Fiber}

Next three dimensional numerical experiment, investigates a composite matrix reinforced with continuous boron fibers which was previously studied in [47]. Same as other examples, a proper RVE is considered as the domain of interest. Distance field functions are generated for each phase of the structure and after creation of 


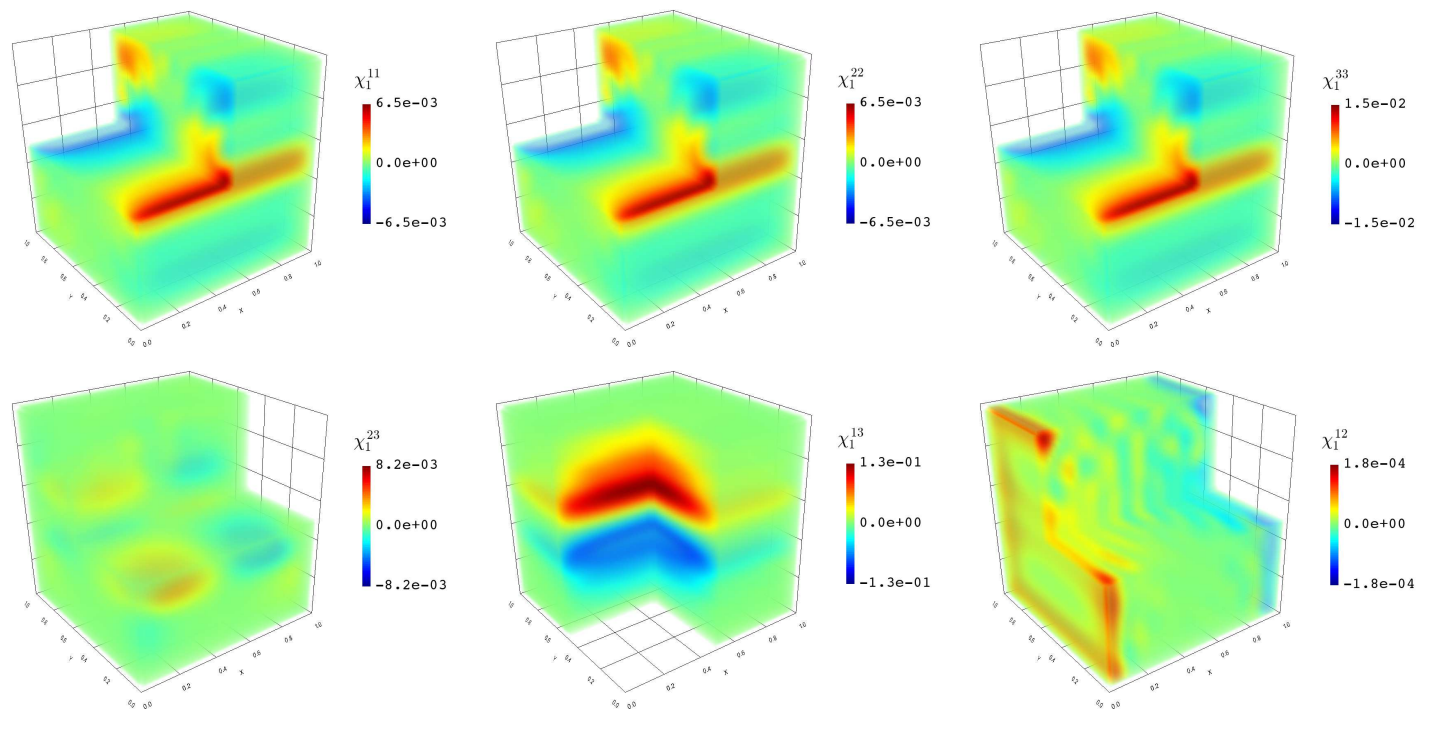

Figure 6.3: $\chi_{1}^{k l}$ functions for layered composite.

Table 6.2: Homogenized stiffness matrix of the layered composite RVE.

\begin{tabular}{lccc}
\hline & MeshFree/Asymptotic & Strain Energy Method & Difference \\
\hline$E_{1111}^{H}$ & 21.41 & 21.18 & $1.09 \%$ \\
$E_{2222}^{H}$ & 21.41 & 21.18 & $1.09 \%$ \\
$E_{3333}^{H}$ & 9.46 & 8.81 & $7.38 \%$ \\
$E_{1122}^{H}$ & 8.19 & 8.03 & $1.99 \%$ \\
$E_{1133}^{H}$ & 4.64 & 4.33 & $7.16 \%$ \\
$E_{2233}^{H}$ & 4.64 & 4.33 & $4.10 \%$ \\
$E_{2323}^{H}$ & 3.05 & 2.93 & $4.10 \%$ \\
$E_{1313}^{H}$ & 3.05 & 2.93 & $4.10 \%$ \\
$E_{1212}^{H}$ & 6.60 & 6.65 & $0.75 \%$ \\
\hline
\end{tabular}




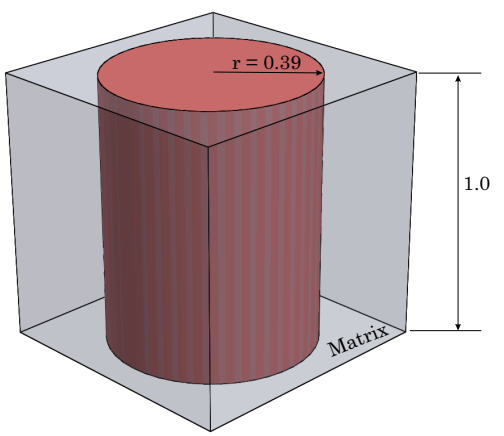

Figure 6.4: RVE geometry for a composite structure with fiber reinforcement, $V_{f}$ (fiber volume fraction) $=0.47$ ).

Table 6.3: Mechanical properties of matrix and fiber [47].

\begin{tabular}{ll}
\hline Property & Value \\
\hline Matrix elastic modulus, $E_{m}(\mathrm{GPa})$ & 68.3 \\
Matrix Poisson's ratio, $\nu_{m}$ & 0.30 \\
Reinforcement elastic modulus, $E_{m}(\mathrm{GPa})$ & 379.3 \\
Reinforcement Poisson's ratio, $\nu_{m}$ & 0.10 \\
\hline
\end{tabular}

the nonconforming grid and distribution of Gauss integration points, $\chi$ functions are calculated. Fig. 6.4 and Table 6.3 respectively show the RVE structure and mechanical properties corresponding to each material in the domain. Distance field functions and a section of Gauss integration point distribution are presented in Fig. 6.5 and Fig. 6.6 shows the $\chi$ functions. Considering the fact that the behaviour of structure in $x$ and $y$ directions are the same, only one of the two sets of $\chi$ functions corresponding to $x$ and $y$ directions $(m=1,2)$ are plotted alongside with the ones related to $z$ direction $(m=3)$ and eventually homogenized properties are tabulated in Table 6.4. Reviewing homogenized properties computed via MeshFree/Asymptotic platform shows quite satisfactory agreement with conventional finite element results, with the maximum difference of $3 \%$. 


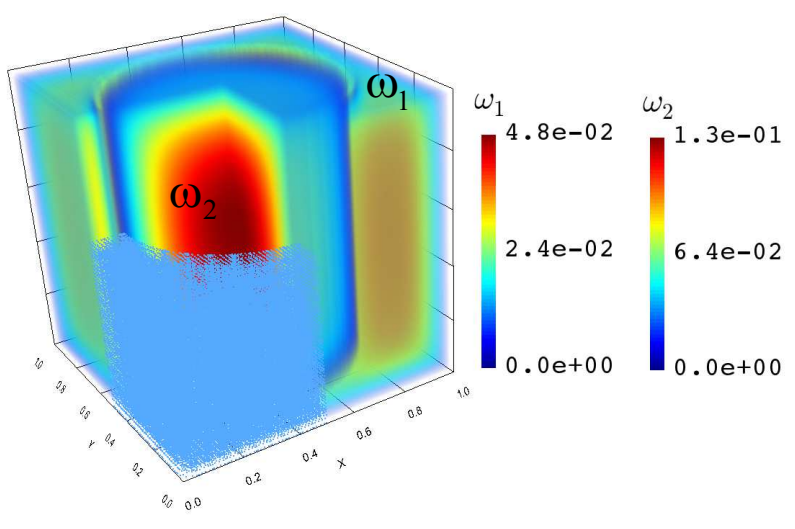

Figure 6.5: Distance field functions for matrix and reinforcement fiber and Gauss integration point distribution in a section of domain.

Table 6.4: Homogenized stiffness matrix of the composite with fiber reinforcement.

\begin{tabular}{lccc}
\hline & MeshFree/Asymptotic & Strain Energy Method [47] & Differences \\
\hline$E_{1111}^{H}$ & 181.47 & 178.21 & $1.83 \%$ \\
$E_{2222}^{H}$ & 181.47 & 178.21 & $1.83 \%$ \\
$E_{3333}^{H}$ & 231.56 & 231.04 & $0.23 \%$ \\
$E_{1122}^{H}$ & 44.48 & 45.27 & $1.75 \%$ \\
$E_{1133}^{H}$ & 40.68 & 40.49 & $0.47 \%$ \\
$E_{2233}^{H}$ & 40.68 & 40.49 & $0.47 \%$ \\
$E_{2323}^{H}$ & 74.93 & 73.19 & $2.38 \%$ \\
$E_{1313}^{H}$ & 74.93 & 73.19 & $2.38 \%$ \\
$E_{1212}^{H}$ & 64.89 & 62.80 & $3.33 \%$ \\
\hline
\end{tabular}




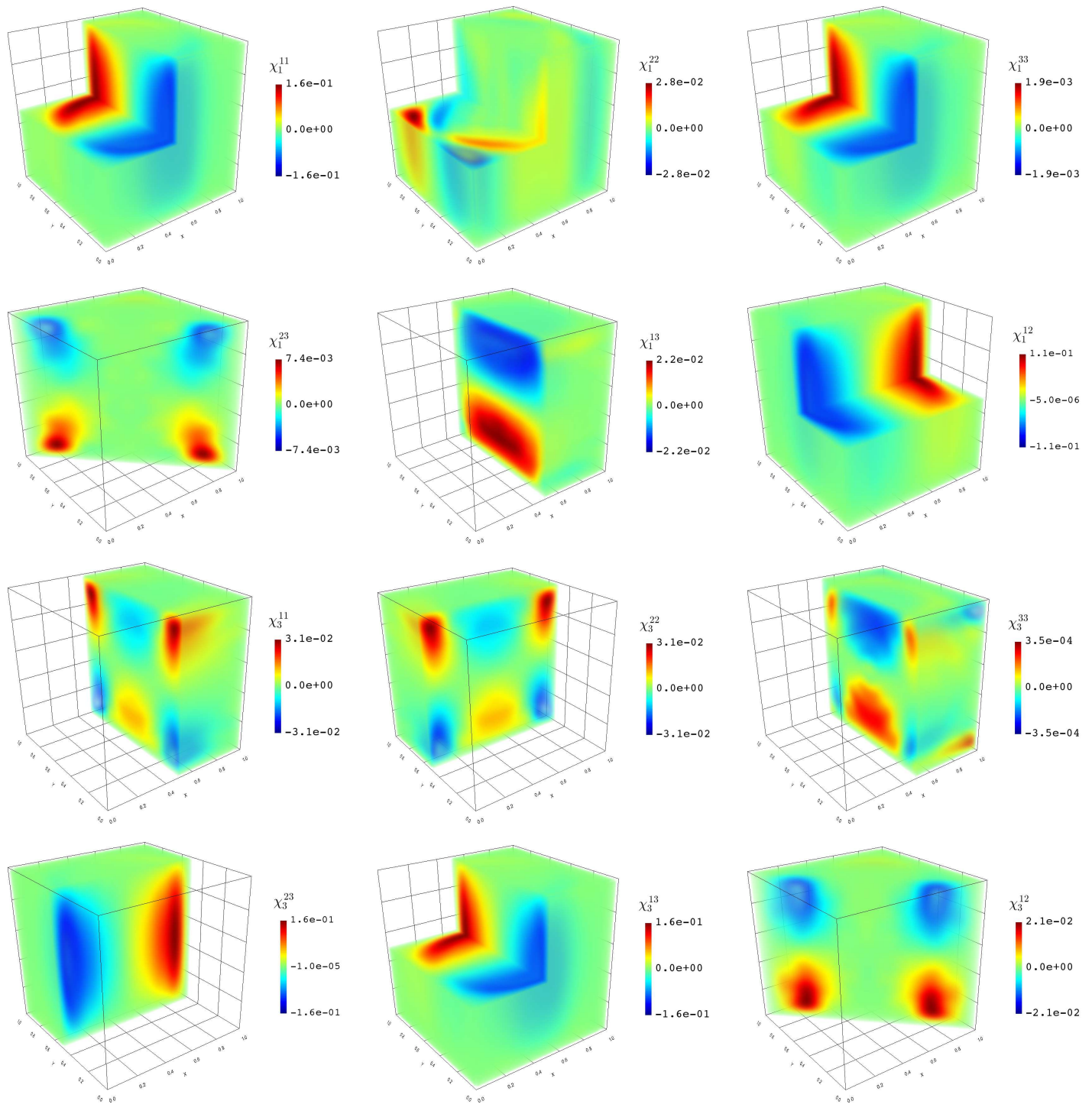

Figure 6.6: $\chi_{m}^{k l}$ functions for composite with fiber reinforcement. 


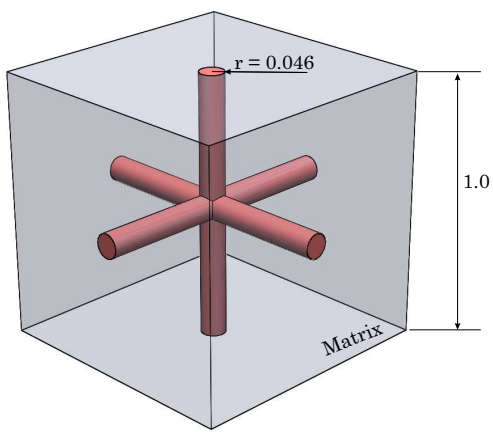

Figure 6.7: RVE geometry for a composite structure with reinforcement grid, $V_{f}$ (fiber volume fraction $)=0.02$.

\subsection{Cubic Grid-Reinforced Composite with Orthotropic Re- inforcement}

As the last example, a cubic RVE containing fiber reinforcements in three directions which is studied in [33] is considered as the source of domain geometry. Fig. 6.7 shows the RVE structure and mechanical properties for matrix and reinforcements are presented in Table 6.5 which clearly shows the orthotropic behavior of the reinforcement structure. Distance field functions and Gauss integration point distribution are shown in Fig. 6.8 and Table 6.6 compares the homogenized properties computed via MeshFree/Asymptotic platform and conventional finite elements side by side.

Reviewing the numerical experiments presented in Chapter 3 on 2D domains and the ones investigated in this chapter for 3D RVEs, we can clearly see that the MeshFree/Asymptotic method has a very satisfactory performance and provides a robust and fully automated platform to compute the homogenized properties of various inhomogeneous structures. 
Table 6.5: Mechanical properties of matrix and fiber [33].

\begin{tabular}{ll}
\hline Property & Value \\
\hline Matrix elastic modulus, $E_{m}(\mathrm{GPa})$ & 3.19 \\
Matrix Poisson's ratio, $\nu_{m}$ & 0.35 \\
Reinforcement Properties & \\
$E_{1}(\mathrm{GPa})$ & 173.06 \\
$E_{2}(\mathrm{GPa})$ & 33.06 \\
$E_{3}(\mathrm{GPa})$ & 5.17 \\
$G_{23}(\mathrm{GPa})$ & 3.24 \\
$G_{13}(\mathrm{GPa})$ & 8.27 \\
$G_{12}(\mathrm{GPa})$ & 9.37 \\
$\nu_{23}$ & 0.17 \\
$\nu_{13}$ & 0.25 \\
$\nu_{12}$ & 0.03 \\
\hline
\end{tabular}

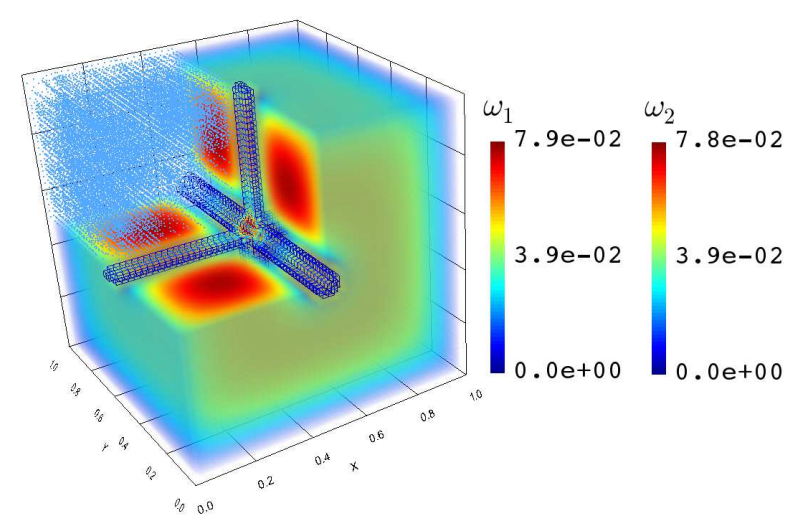

Figure 6.8: Distance field functions for matrix and reinforcement grid and Gauss integration point distribution in a section of domain. 
Table 6.6: Homogenized stiffness matrix of the composite with reinforcement grid.

\begin{tabular}{lccc}
\hline & MeshFree/Asymptotic & Strain Energy Method & Differences \\
\hline$E_{1111}^{H}$ & 6.45 & 6.35 & $1.57 \%$ \\
$E_{2222}^{H}$ & 5.42 & 5.38 & $0.74 \%$ \\
$E_{3333}^{H}$ & 5.10 & 5.12 & $0.39 \%$ \\
$E_{1122}^{H}$ & 2.75 & 2.76 & $0.36 \%$ \\
$E_{1133}^{H}$ & 2.73 & 2.74 & $0.36 \%$ \\
$E_{2233}^{H}$ & 2.72 & 2.74 & $0.73 \%$ \\
$E_{2323}^{H}$ & 1.21 & 1.20 & $0.83 \%$ \\
$E_{1313}^{H}$ & 1.24 & 1.22 & $1.64 \%$ \\
$E_{1212}^{H}$ & 1.24 & 1.22 & $1.64 \%$ \\
\hline
\end{tabular}




\section{CHAPTER 7 \\ CONCLUSION}

\section{Summary and Discussion}

In this dissertation a novel computational method is introduced to calculate homogenized mechanical properties of materials with inhomogeneous structures. This method combines Asymptotic Homogenization technique with MeshFree Solution Structures and creates a hybrid system which inherits the perks of both meshfree and asymptotic theories.

Theoretical background and mathematical formulation of asymptotic homogenization and meshfree solution structures are discussed in detail. It is also explained how these two methods are combined. Then a computational platform is developed based on the hybrid Meshfree/Asymptotic method. This platform takes advantage of a meshfree geometric engine which converts micrograph images of complex structures into models suitable for numerical analysis.

Solving homogenization problem using conventional finite elements requires generation of a geometry conforming mesh over the domain, and when the geometric information of this domain is provided by only images, first these image data need to be converted to finite element meshes. There are very few software packages to perform the latter, they create a preliminary mesh over the domain and provide the user with a group of tools to manually apply them and modify the mesh to get to the desired quality. In addition, for boundary value problems with periodic boundary conditions (e.g. homogenization problem) the modified mesh requires additional treatments such as node pairing to be suitable for implementation of such boundary conditions. 


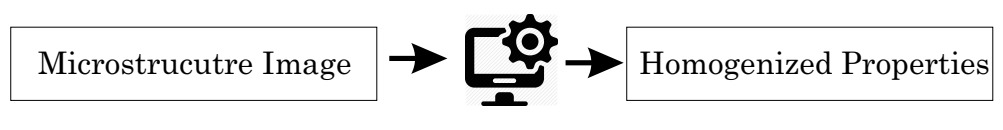

MeshFree/Asymptotic

Platform

Figure 7.1: MeshFree/Asymptotic Platform

While in the developed platform, meshfree modeling of the domain simplifies meshing to generation of a simple cartesian grid which only needs to contain the domain. Also a suitable solution structure is designed which provides exact satisfaction of periodic boundary conditions. Consequently there would be no need for any manual mesh modifications and this will provide a completely automated platform for computing homogenized mechanical properties (see Fig. 7.1).

This platform is created in conjunction with a larger meshfree library in $\mathrm{C}++$ and necessary modulus and features are designed and added to it. One of the novel features developed in this work is a Meshfree Solution Structure for boundary value problems with Periodic Boundary Conditions. This structure utilizes a group of carefully designed enrichment functions to enforce periodicity conditions on the boundaries and provide Exact Satisfaction of periodic boundary conditions.

Performance of the developed platform is tested with numerical experiments in two major categories: geometries presented as CAD models and geometries captured from images. In all cases where RVE geometry is presented in form of a CAD model, meshfree/asymptotic results and previously published data are very similar, with the maximum difference of $11 \%$. Where micrograph images are directly imported to the platform, diagonal elements of homogenized elasticity tensor obtained from meshfree/asymptotic and strain energy methods are again in a very good agreement (within 10\% of each other). Discrepancies among off-diagonal elements are more noticeable, and the reasons behind them originates from theoretical differences between meshfree/asymptotic and strain energy methods. 
As stated earlier, there are very few software packages available to create finite element models from image data and among those available as open source platforms, OOF2 is the one used to compute benchmark properties. Although OOF2/strain energy method does not use the same approaches as meshfree/asymptotic method for geometry modeling, solving the homogenization problem and post processing the results, it is still one of the best numerical benchmarks available.

The developed platform is capable of handling multi-phase inhomogeneous domains and producing fully anisotropic homogenized elasticity tensor. It also captures the realistic geometry from images and in a completely automated process computes the homogenized properties. Results of meshfree/asymptotic method are in the same level of accuracy as available methods such as strain energy, but due to elimination of meshing and manual mesh modifications these results are less expensive.

Fig 7.2 compares the solution times for computing homogenized properties of a sample RVE (see Section 3.2.3) with meshfree and conventional finite elements. Solution time which consists of: Importing the Geometry \& Preparing the Model, Solving Homogenization Problem and Post Processing the Results is almost the same for both methods. The main difference is that in conventional finite element method the time spent for preparing the model is a person's time, but meshfree methods do the same thing automatically by consuming more processing power which is much cheaper than a skilled finite element user. It also worth mentioning, currently the developed $\mathrm{C}++$ code runs on a single node and it can be easily modified for parallel computing which significantly reduces solution time.

After satisfactory evaluation of the developed platform in predicting homogenized properties of inhomogeneous structures, those properties are used to find the localized distribution of stresses and strains in nonuniform domains. Three different numerical experiments were designed using previously studied RVEs. Boundary 


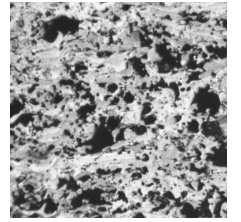

(a)

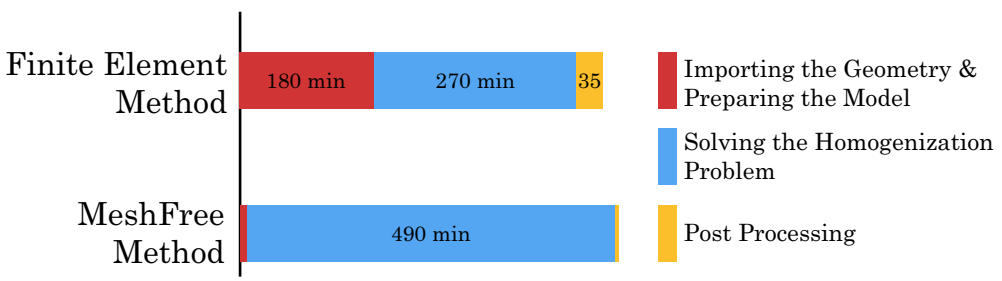

(b)

Figure 7.2: (a) RVE for Al-10-CNT, (b) Solution time comparison for conventional finite elements and meshfree solution structures. The time for importing geometry \& preparing the model is the time spent by a skilled finite element user to import Fig. 3.34(b) into OOF2 and create the mesh shown in Fig. 3.37.

conditions and loads simulating real engineering problems are applied to these domains and using $L_{2}$-norms of displacements, convergence of the localized values to the results of full geometric models are investigated. For $\epsilon<<1$, localized values of displacements are within $2 \%$ of full geometric model results. Also localized stress distribution patterns are very well predicted and stress concentration zone are clearly distinguishable.

Although error distribution plots for stresses show high error values, these spikes are limited to finite number of boundary points. In areas where RVE boundaries at local scale coincides with global boundaries, local solution structure enforces periodic boundary conditions at local scale and this can cause noises on global responses.

Furthermore a study is conducted on the effect of geometric features on homogenized mechanical properties. It is shown that in addition to volume fraction of porosities/inclusions (which currently is the major factor determining homogenized properties in most of homogenization techniques), their concentration and spatial orientation also plays an important role in homogenized properties.

More importantly, it is demonstrated that automated calculation of homogenized properties provides the opportunity for Automated Quantitative Analysis of homogenized properties, which could be a powerful design and optimization tool. 
For example, 3D printing technology gives user the ability to treat material properties, their volume fractions and microstructure geometry as design variables for reaching to a desired property. In such applications, a quantitative analysis of geometric features effect on homogenized properties would lead to a more optimized and cost effective designs.

At the end, the developed platform was equipped with necessary modulus to be able to work with 3D domains. This will allow us to use computer generated models as geometric representation of the material structure and calculate its homogenized mechanical properties. Performance of the 3D block was tested against previously published data and in all numerical experiments promising results were obtained $(\max$ error $<7 \%)$.

\section{Limitations and Future Works}

- Currently the solution structure satisfying periodic boundary conditions is developed for rectangular or cubical RVEs. Developing similar structures for RVEs with different geometries such as triangle and tetrahedral could provide more flexibility in handling complex geometries.

- Asymptotic method works on the key assumption that the material microstructure is periodic, studying effect of different RVE selections could improve performance of the developed method over non-periodic domains.

- At the moment geometric block of our platform can only convert 2D images into distance field function representations. For studying 3D domains only CAD models can be used. Improving the geometric engine to convert 3D image data into distance field functions would extend applications of meshfree/asymptotic method. For example this method could be applied to MRI images of structures such as bones to study their properties. 
- The Current version of meshfree/asymptotic platform uses LU decomposition method from PETSc library for solving system of linear algebraic equations, for example eq. (3.9). This library contains other direct and itterative solvers. Using more advanced solvers could increase efficiency of the platform.

- Homogenized elasticity tensor is currently presented as constant values. Improving computational efficiency of our platform would provide the tools to divide a single domain into more than one RVE, and compute homogenized properties at different sections of material and eventually provide a functionally graded response. 


\section{BIBLIOGRAPHY}

[1] M. Anderson and Q. Ku. Process of making porous ceramic materials with controlled porosity, July 13 1993. US Patent 5,227,342.

[2] C. Armando Duarte, D.-J. Kim, and I. Babuka. A global-local approach for the construction of enrichment functions for the generalized fem and its application to three-dimensional cracks. In V. Leito, C. Alves, and C. Armando Duarte, editors, Advances in Meshfree Techniques, volume 5 of Computational Methods in Applied Sciences, pages 1-26. Springer Netherlands, 2007.

[3] S. R. Bakshi, A. Bhargava, S. Mohammadizadeh, A. Agarwal, and I. Tsukanov. Computational estimation of elastic properties of spark plasma sintered tac by meshfree and finite element methods. Computational Materials Science, 50(9):2615 - 2620, 2011.

[4] M. P. Bendsøe and N. Kikuchi. Generating optimal topologies in structural design using a homogenization method. Computer methods in applied mechanics and engineering, 71(2):197-224, 1988.

[5] Y. Benveniste. A new approach to the application of Mori-Tanaka's theory in composite materials. Mechanics of Materials, 6(2):147-157, 1987.

[6] A. Biswas and V. Shapiro. Approximate distance fields with non-vanishing gradients. Graphical Models, 66(3):133 - 159, 2004.

[7] P. Cartraud and T. Messager. Computational homogenization of periodic beamlike structures. International Journal of Solids and Structures, 43(3-4):686-696, Feb. 2006.

[8] P. W. Chung, K. K. Tamma, and R. R. Namburu. Asymptotic expansion homogenization for heterogeneous media: computational issues and applications. Composites Part A: Applied Science and Manufacturing, 32(9):12911301, 2001.

[9] V. R. Coffman, A. C. Reid, S. A. Langer, and G. Dogan. OOF3D: An imagebased finite element solver for materials science. Mathematics and Computers in Simulation, 82(12):2951-2961, Aug. 2012.

[10] U. Congress. Advanced materials by design. Technical report, Office of Technology Assessment, June 1988. 
[11] M. Freytag, V. Shapiro, and I. Tsukanov. Field modeling with sampled distances. Computer-Aided Design, 38(2):87-100, Feb. 2006.

[12] M. Freytag, V. Shapiro, and I. Tsukanov. Finite element analysis in situ. Finite Elements in Analysis and Design, 47(9):957-972, Sept. 2011.

[13] M. K. Freytag, V. Shapiro, and I. Tsukanov. Scan and Solve: Acquiring the physics of artifacts. In ASME 2007 International Design Engineering Technical Conferences and Computers and Information in Engineering Conference, pages 345-356. American Society of Mechanical Engineers, 2007.

[14] H. Gans and P. Woodmansee. An improved method of modeling porous materials using finite elements. Computers and Structures, 44(5):1055 - 1063, 1992.

[15] M. Geers, V. Kouznetsova, and W. Brekelmans. Multi-Scale computational homogenization: Trends and challenges. Journal of Computational and Applied Mathematics, 234(7):2175-2182, Aug. 2010.

[16] S. Ghosh, K. Lee, and S. Moorthy. Multiple scale analysis of heterogeneous elastic structures using homogenization theory and voronoi cell finite element method. International Journal of Solids and Structures, 32(1):27 - 62, 1995.

[17] J. M. Guedes and N. Kikuchi. Preprocessing and postprocessing for materials based on the homogenization method with adaptive finite element methods. Computer Methods in Applied Mechanics and Engineering, 83:143-198, 1990.

[18] Z. Hashin and S. Shtrikman. A variational approach to the theory of the effective magnetic permeability of multiphase materials. Journal of Applied Physics, 33(10):3125-3133, oct 1962.

[19] Z. Hashin and S. Shtrikman. A variational approach to the theory of elastic behaviour of multiphase materials. Journal of the Mechanics and Physics of Solids, 11(2):127-140, apr 1963.

[20] B. Hassani and E. Hinton. A review of homogenization and topology opimization I-analytical and numerical solution of homogenization equations. Computers $\& 5$ Structures, 69(6):707-717, 1998.

[21] B. Hassani and E. Hinton. A review of homogenization and topology opimization II-analytical and numerical solution of homogenization equations. Computers $\& 3$ structures, 69(6):719-738, 1998. 
[22] B. Hassani and E. Hinton. A review of homogenization and topology optimization iiitopology optimization using optimality criteria. Computers 85 Structures, 69(6):739-756, 1998.

[23] S. Hazanov. Hill condition and overall properties of composites. Archive of Applied Mechanics, 68(6):385-394, 1998.

[24] S. Hazanov and M. Amieur. On overall properties of elastic heterogeneous bodies smaller than the representative volume. International Journal of Engineering Science, 33(9):1289 - 1301, 1995.

[25] S. Hazanov and C. Huet. Order relationships for boundary conditions effect in heterogeneous bodies smaller than the representative volume. Journal of the Mechanics and Physics of Solids, 42(12):1995 - 2011, 1994.

[26] R. Hill. Elastic properties of reinforced solids: Some theoretical principles. Journal of the Mechanics and Physics of Solids, 11(5):357 - 372, 1963.

[27] R. Hill. A self-consistent mechanics of composite materials. Journal of the Mechanics and Physics of Solids, 13:213-222, 1965.

[28] S. J. Hollister and N. Kikuchi. A comparison of homogenization and standard mechanics analyses for periodic porous composites. Computational Mechanics, 10(2):73-95, 1992.

[29] A. Iske. On the construction of mass conservative and meshless adaptive particle advection methods. In V. Leito, C. Alves, and C. Armando Duarte, editors, Advances in Meshfree Techniques, volume 5 of Computational Methods in Applied Sciences, pages 169-186. Springer Netherlands, 2007.

[30] M. Jiang, K. Alzebdeh, I. Jasiuk, and M. Ostoja-Starzewski. Scale and boundary conditions effects in elastic properties of random composites. Acta Mechanica, 148(1-4):63-78, 2001.

[31] Y. Jiang, W. Guo, and H. Yang. Numerical studies on the effective shear modulus of particle reinforced composites with an inhomogeneous inter-phase. Computational Materials Science, 43(4):724-731, Oct. 2008.

[32] J. M. Jon Binner, Paul Hogg. Advanced Materials Source Book. Elsevier, October 2013. 
[33] A. Kalamkarov, E. Hassan, A. Georgiades, and M. Savi. Asymptotic homogenization model for $3 \mathrm{~d}$ grid-reinforced composite structures with generally orthotropic reinforcements. Composite Structures, 89(2):186 - 196, 2009.

[34] A. L. Kalamkarov, I. V. Andrianov, and V. V. Danishevs'kyy. Asymptotic homogenization of composite materials and structures. Applied Mechanics Reviews, 62(3):1-20, 2009.

[35] P. Kanout, D. Boso, J. Chaboche, and B. Schrefler. Multiscale methods for composites: A review. Archives of Computational Methods in Engineering, 16(1):31-75, 2009.

[36] L. V. Kantorovich and V. I. Krylov. Approximation methods of higher analysis. Interscience, New York, 1958.

[37] T. Kosta and I. Tsukanov. Meshfree natural vibration analysis of $2 \mathrm{~d}$ structures. Computational Mechanics, 53(2):283-296, 2014.

[38] S. A. Langer, E. R. Fuller Jr, and W. C. Carter. OOF: An image-based finiteelement analysis of material microstructures. Computing in Science 85 Engineering, 3(3):15-23, 2001.

[39] B. Luft, V. Shapiro, and I. Tsukanov. Geometrically adaptive numerical integration. In Proceedings of 2008 ACM Symposium on Solid and Physical Modeling, pages 147-157, Stony Brook, NY, June 2008.

[40] D. Lukkassen, L. E. Persson, and P. Wall. Some engineering and mathematical aspects on the homogenization method. Composites Engineering, 5(5):519-531, 1995.

[41] B. I. Lundqvist, A. Bogicevic, S. Dudiy, P. Hyldgaard, S. Ovesson, C. Ruberto, E. Schröder, and G. Wahnström. Bridging between micro-and macroscales of materials by mesoscopic models. Computational Materials Science, 24(1):1-13, 2002.

[42] P. R. Marur. Numerical estimation of effective elastic moduli of syntactic foams. Finite Elements in Analysis and Design, 46(11):1001-1007, Nov. 2010.

[43] J. Mathias and N. Tessier-Doyen. Homogenization of glass/alumina two-phase materials using a cohesive zone model. Computational Materials Science, 43(4):1081-1085, Oct. 2008. 
[44] T. Mori and K. Tanaka. Average stress in matrix and average elastic energy of materials with misfitting inclusions. Acta Metallurgica, 21:571-574, may 1973.

[45] V.-D. Nguyen, E. Bchet, C. Geuzaine, and L. Noels. Imposing periodic boundary condition on arbitrary meshes by polynomial interpolation. Computational Materials Science, 55(0):390 - 406, 2012.

[46] J. Oden and K. S. Vemaganti. Estimation of local modeling error and goaloriented adaptive modeling of heterogeneous materials: I. error estimates and adaptive algorithms. Journal of Computational Physics, 164(1):22 - 47, 2000.

[47] J. Oliveira, J. Pinho-da Cruz, and F. Teixeira-Dias. Asymptotic homogenisation in linear elasticity. part II: Finite element procedures and multiscale applications. Computational Materials Science, 45(4):1081-1096, June 2009.

[48] M. Ostoja-Starzewski. Material spatial randomness: From statistical to representative volume element. Probabilistic Engineering Mechanics, 21(2):112 $132,2006$.

[49] R. Pampuch. Ceramic Materials: An Introduction to Their Properties. Amsterdam: Elsevier Scientific Pub. Co, 1976.

[50] J. Pinho-da Cruz, J. Oliveira, and F. Teixeira-Dias. Asymptotic homogenisation in linear elasticity. Part I: Mathematical formulation and finite element modelling. Computational Materials Science, 45(4):1073-1080, June 2009.

[51] Y. Prawoto, J. Djuansjah, and N. Shaffiar. Re-visiting the 'Rule of Mixture' used in materials with multiple constituting phases: A technical note on morphological considerations in austenite case study. Computational Materials Science, 65:528-535, Dec. 2012.

[52] L. B. Rall and G. F. Corliss. An introduction to automatic differentiation. In M. Berz, C. H. Bischof, G. F. Corliss, and A. Griewank, editors, Computational Differentiation: Techniques, Applications, and Tools, pages 1-17. SIAM, Philadelphia, PA, 1996.

[53] V. Rvachev. Theory of R-functions and some applications. Naukova Dumka, Kiev, 1982.

[54] V. Rvachev and T. Sheiko. R-functions in boundary value problems in mechanics. Applied Mechanics Review, 48(4):38, April 1995. 
[55] V. Rvachev, T. Sheiko, V. Shapiro, and I. Tsukanov. Transfinite interpolation over implicitly defined sets. Computer Aided Geometric Design, 18:195-220, 2001.

[56] V. L. Rvachev, T. I. Sheiko, V. Shapiro, and I. Tsukanov. On completeness of RFM solution structures. Computational Mechanics, 25(2-3):305-317, 2000.

[57] T. Saito and J.-I. Toriwaki. New algorithms for euclidean distance transformation of an n-dimensional digitized picture with applications. Pattern Recognition, 27(11):1551 - 1565, 1994.

[58] V. Shapiro. Real functions for representation of rigid solids. Computer Aided Geometric Design, 11(2):153 - 175, 1994.

[59] V. Shapiro and I. Tsukanov. Implicit functions with guaranteed differential properties. In Proceedings of the fifth ACM symposium on Solid modeling and applications, pages 258-269. ACM, 1999.

[60] A. Shevchenko and V. Rokityanskaya. Automatic differentiation of functions of many variables. Cybernetics and Systems Analysis, 32(5):709-724, 1996.

[61] F. Tang, H. Fudouzi, T. Uchikoshi, and Y. Sakka. Preparation of porous materials with controlled pore size and porosity. Journal of the European Ceramic Society, 24(2):341 - 344, 2004. 8th International Conference on Ceramic Processing.

[62] I. Tsukanov and M. Hall. Fast forward automatic differentiation library (ffadlib): A user manual. Technical report 2000-4, Spatial Automation Laboratory, University of Wisconsin-Madison, December 2000.

[63] I. Tsukanov and M. Hall. Data structure and algorithms for fast automatic differentiation. International Journal for Numerical Methods in Engineering, 56:1949-1972, 2003.

[64] I. Tsukanov and V. Shapiro. The architecture of SAGE-a meshfree system based on RFM. Engineering with Computers, 18(4):295-311, 2002.

[65] I. Tsukanov and V. Shapiro. Meshfree modeling and analysis of physical fields in heterogeneous media. Advances in Computational Mathematics, 23(1-2):95124, 2005. 
[66] I. Tsukanov, V. Shapiro, and S. Zhang. A meshfree method for incompressible fluid dynamics problems. International Journal for Numerical Methods in Engineering, 58(1):127-158, 2003.

[67] G. Turk and J. F. O'Brien. Modelling with implicit surfaces that interpolate. ACM Trans. Graph., 21(4):855-873, Oct. 2002.

[68] J. Tyrus, M. Gosz, and E. DeSantiago. A local finite element implementation for imposing periodic boundary conditions on composite micromechanical models. International Journal of Solids and Structures, 44(9):2972 - 2989, 2007.

[69] G. Wang, S. Li, H.-N. Nguyen, and N. Sitar. Effective elastic stiffness for periodic masonry structures via eigenstrain homogenization. Journal of materials in civil engineering, 19(3):269-277, 2007.

[70] Q.-S. Yang and W. Becker. Numerical investigation for stress, strain and energy homogenization of orthotropic composite with periodic microstructure and nonsymmetric inclusions. Computational Materials Science, 31(1-2):169-180, Sept. 2004.

[71] H.-K. Zhao, S. Osher, and R. Fedkiw. Fast surface reconstruction using the level set method. In Variational and Level Set Methods in Computer Vision, 2001. Proceedings. IEEE Workshop on, pages 194-201, 2001.

[72] T. I. Zohdi, J. Oden, and G. J. Rodin. Hierarchical modeling of heterogeneous bodies. Computer Methods in Applied Mechanics and Engineering, 138(14):273 - 298, 1996. 
VITA

\section{SIAVASH RASTKAR}

2006

B.S., Mechanical Engineering Sharif University of Technology Tehran, Iran

2009

M.S., Mechanical Engineering Sharif University of Technology Tehran, Iran

2016

Ph.D., Mechanical Engineering Florida International University Miami, Florida

\section{PUBLICATIONS AND PRESENTATIONS}

A. Abdoli, G. S. Dulikravich, G. Vasquez and S. Rastkar, Thermo Fluid Stress Deformation Analysis of Two Layer Microchannels for Cooling Chips with Hot Spots, ASME Journal of Electronic Packaging, Vol. 137, No. 3, pp. 2015.

S. Rastkar, D. Quintero, D. Bolivar, S. Tosunoglu, Empowering Robots via Cloud Robotics: Image Processing and Decision Making BoeBots, Proceedings of the 25th Florida Conference on Recent Advances in Robotics, FCRAR 2012, Boca Raton, Florida, May 10 11, 2012. 